\title{
Characterizing Variability of Audiovisual Speech Perception Based on Periodic and Aperiodic Features of Prestimulus Brain Activity
}

\author{
Vinsea A V Singh ${ }^{1}$, Vinodh G Kumar ${ }^{1,2}$, Arpan Banerjee ${ }^{1}$, Dipanjan Roy ${ }^{1,3^{*}}$
}

1. Cognitive Brain Dynamics Lab, National Brain Research Centre, NH-8, Manesar, Gurugram, 122052, India.

2. Department of Neurology, Penn State College of Medicine Hershey, Pennsylvania, 17033, U.S.A.

3. School of AIDE, Center for Brain Research and Applications, Indian Institute of Technology (IIT) Jodhpur 342037, India.

* Corresponding author. Email: droy@iitj.ac.in 


\section{Abstract}

Burgeoning pieces of evidence support the idea that differences in the properties of large-scale cortical networks underlie perceptual variability. Here, employing psychophysical experiments in tandem with electroencephalogram (EEG) recordings, we explored the inter-individual and inter-trial differences in McGurk percept to probe the underlying brain dynamics before (prestimulus) and after (poststimulus) the stimulus presentation. Initially, based on our participants' $(\mathrm{n}=18)$ overall susceptibility to the McGurk effect, we categorized them into two groups - 'rare' and 'frequent' groups of perceivers. Subsequently, we investigated the differences in their neuronal dynamics manifested in the oscillatory properties of the prestimulus and compared it with the poststimulus EEG signals during the McGurk perception (audio- $/ \mathrm{pa} /$, video- $/ \mathrm{ka} /$ ) and, also during the perception of the congruent audiovisual (AV) stimuli (syllables /pa/, /ta/, and / $k a /$ ). We primarily focused on the power spectrum components (periodic oscillations and aperiodic components) and global coherence. At the inter-individual and inter-trial level, we observed that for the prestimulus duration, the 'rare' perceivers' aperiodic (or "background" 1/f) component elicited a lower beta band (15-30 Hz) and gamma band $(31-45 \mathrm{~Hz})$ activity than the 'frequent' group of perceivers. Moreover, the periodic oscillations for the 'rare' group showed significance in the alpha $(8-12 \mathrm{~Hz})$ and beta bands during the prestimulus time window. 'Rare' perceivers also showed an increased global coherence in the theta (4-7 Hz), alpha, and beta bands but a lower gamma band activity than the 'frequent' perceivers. Finally, 'rare' perceivers elicited higher cross-hemispheric connectivity (imaginary coherence) than the 'frequent' group at the source across different frequency bands, supporting the notion that the fluctuations in the distributed large-scale network underlie the perceptual variability in speech across individuals. 
Keywords: Multisensory, prestimulus, McGurk, EEG, periodic oscillations, aperiodic components, global coherence, source connectivity, imaginary coherence.

\section{Introduction}

Perception is governed by the incoming stimulus and by one's internal brain states (Jensen O. et al., 2012). Numerous studies have highlighted the importance of studying brain activity elicited before the stimulus onset to understand individual and trial-wise variability of sensory perception and predicting individual's predisposition towards upcoming stimulus category (Stephanie J. Kayser et al., 2016; Spadone and Croce et al., 2020; Dijk and Jensen et al., 2008). One such modality where studying the prestimulus brain activity might be beneficial in understanding the source of statespecific modulation of perception is multisensory speech processing. Speech perception, especially during face-to-face conversations, requires temporal integration between auditory and visual inputs in a specific set of brain regions. The encoding, decoding, and perceiving different sensory modalities during speech perception involves multisensory integration (MSI). To efficaciously understand the MSI phenomenon, one can study the brain dynamics of the participants subjected to the McGurk effect (McGurk and MacDonald, 1976). McGurk illusion is perceived when audio of one syllable (for example, $/ b a /$ ) is dubbed onto a video of lip movements articulating another syllable (for example, $/ g a /)$, the individual perceives a phantom syllable (in this case / $d a /$ ). Studies have reported variability among participants perceiving the McGurk illusion. This variability arises because some participants experience the McGurk illusion more frequently than others (MMK Benoit et al., 2010; Nath \& Beuchamp, 2012; Strand JF et al., 2014; Mallick et al., 2015; Kumar et al., 2020). 
Moreover, an individual perceives the same illusion on some occasions (experimental trials) but not in others exhibiting inter-trial differences. This across-individuals and within-subject variability in McGurk illusory perception have aided researchers in determining the neural correlates of crossmodal percept in terms of neural oscillations and cortical activations (Keil, Müller, Ihssen, \& Weisz, 2012; Nath, Fava \& Beauchamp, 2011). Furthermore, large-scale functional connectivity estimates between multisensory speech centers and through computational modelling have been inferred that the specific inter-individual variability of McGurk susceptibility may critically explain the emergent perceptual and neural differences between groups of perceivers (Kumar et al., 2020).

Furthermore, some studies have suggested that the variability seen across participants may be due to an individual's ongoing oscillatory brain activity, which is already present in the absence of any stimulus (defined as the prestimulus brain state or activity). In this case, the susceptibility to the McGurk illusion, the response to the upcoming stimulus can be influenced by prestimulus neuronal dynamics (e.g., the phase, amplitude and power of ongoing brain activity). Earlier seminal studies have shed some insight into how prestimulus oscillatory brain activity may sculpt perception and reflect preparatory stages for the incoming McGurk stimulus (Keil, Müller, Ihssen, \& Weisz, 2012). They have reported that activity primarily in the beta band power $(14-30 \mathrm{~Hz})$ in distributed cortical regions, particularly in ISTG (left Superior Temporal Gyrus), can predict the perception of the McGurk effect of the observers. 1STG is one of the key sites involved in speech and language processing and multisensory integration.

However, studies suggest that the neural oscillations one computes and compares with the cognitive, perceptual, and behavioural states do not directly reflect the rhythmic activity (Bullock et al., 2003; 
Buzsáki et al., 2013; He B., 2014; Donoghue et al., 2020; Wen \& Liu, 2016). Rather, in the frequency domain, oscillations manifest as narrowband peaks of power over and above the non-oscillatory (or aperiodic) component. This arrhythmic brain activity has been linked to fluctuations in different cognitive, perceptual, and behavioral states (Engel et al., 2001; Buzsáki \& Draguhn, 2004; He B. et al., 2010; Podvalny et al., 2015). So, the changes in power observed might be because of changes in the aperiodic component, even when no periodic oscillation is present. Due to this, systematic parameterization of the power spectrum is required to circumvent misinterpretation of the observed narrowband power differences between conditions of interest (Cole and Voytek, 2019; Lansbergen et al., 2011). Nonetheless, the parametrized power does not tell us much about the sensor level's whole-brain functional connectivity (FC) dynamics. To compute the large-scale functional connectivity, one can perform global coherence (Cimenser et al., 2011; Balazs et al., 2016; Fonseca et al., 2015; Kumar et al., 2016). Global coherence is a mathematical technique used to capture and quantify the strength of the covariation of neural oscillations at the global (or whole-brain) scale (Railo, Koivisto, \& Revonsuo, 2011). Recent studies have indicated that beyond the specific region of interest, there lies a large-scale network of oscillatory brain networks that govern the McGurk effect (Kumar et al., 2016). These studies have looked at the FC of the whole brain during the stimulus presentation. However, the global coherence measure has not been computed previously to capture large-scale functional connectivity dynamics during the prestimulus duration, eventually leading up to the McGurk percept.

Previous studies have pinpointed specific neural and behavioural differences among participants that were rarely or frequently (participants were characterized by behavioural perceptual responses) (Nath \& Beauchamp, 2012; Kumar et al., 2020) perceiving the McGurk illusion. However, how specifically 
aperiodic and periodic changes in oscillations among different perceivers entail the cognitive component of multisensory integration is not fully understood. Hence, we focused on understanding whether there are specific neural differences across a frequent and rare group of perceivers that are captured by aperiodic and periodic changes in the relevant frequency bands already during their prestimulus brain activity. We hypothesized whether the aperiodic and periodic components of neural oscillatory signatures at the prestimulus stage could differentiate between a rare or a frequent group of McGurk perceivers. To test our hypothesis, we reanalyzed EEG data collected in a previous study that explained the underlying mechanisms governing large-scale brain network dynamics between rare and frequent groups of McGurk perceivers using a biophysical model (Kumar et al., 2020). Firstly, we investigated how the oscillatory (periodic) and the aperiodic component of the power spectrum captures the variability of McGurk perception, and neuronal signatures differ between the two groups of perceivers at inter-individual and inter-trial levels. Subsequently, we computed and analyzed the time-averaged global coherence of the two groups of perceivers to measure the largescale functional connectivity dynamics in the prestimulus duration of both incongruent and congruent AV-stimuli. Finally, we reconstructed the sources and extracted the source time-series to comprehend the nature of functional connectivity among the regions of interest (ROIs) for both the rare and frequent group of perceivers in the prestimulus duration leading to the McGurk effect (a detailed pipeline of different analysis employed is outlined in Figure 1). Based on the findings, this is one of the first studies that pinpoint the specific prestimulus neural differences in both aperiodic and oscillatory components and large-scale functional connectivity among participants that were rarely or frequently perceived multisensory illusory perception. 


\section{Materials and Methods}

\subsection{Participants}

For this study, we have used the previously recorded EEG and behavioural data described in Kumar et al. (2020), where eighteen healthy right-handed participants ( 8 females) with a mean age of $24.9 \pm$ 2.8 years were recruited for the study. They had given written informed consent under the experimental protocol approved by the Institutional Human Ethics Committee (IHEC) of the National Brain Research Centre (NBRC), India, which agrees with the 2008 Declaration of Helsinki.

\subsection{Stimulus Design and Trials}

Digital videos of a native Hindi speaking male articulating the syllables $/ \mathrm{pa} /, / \mathrm{ta} /$, and $/ \mathrm{ka} / \mathrm{were}$ recorded and edited using - the video editing software Videopad Editor (https://www.nchsoftware.com), and the audio editing software Audacity (https://www.audacityteam.org). The duration of auditory syllables articulated in the videos ranged from 0.4 to 0.5 seconds, and the duration of each video clip ranged from 1.5 to 1.7 seconds. Each video included the neutral, mouth closed position, mouth movement of articulation, and mouth closing. The AV-stimuli presented to the participants at random were of four kinds: three congruent (audio syllable matching with the video articulation) syllables /pa/, /ta/, and $/ \mathrm{ka} /$; and one McGurk (audiovisual mismatch) syllable (auditory /pa/with visual / $k a /$ ) producing the illusion of syllable / $\mathrm{ta}$ / (Figure 2). There was 0 lag between the audio and visual input presented to the participants.

The experiment consisted of five blocks- each block was made up of 120 trials (30 trials of each video were presented randomly). Inter-stimulus intervals were pseudo-randomly varied between $1200 \mathrm{~ms}$ (milliseconds) to $2800 \mathrm{~ms}$ to minimize expectancy effects (MW Minor, 1970). Using a forced-choice 
task on every trial, participants reported their subjective perception by pressing a specified key on the keyboard corresponding to $/ p a /, / t a /, / k a /$, or something else while watching the audiovisual videos unaware of the McGurk illusion.

\subsection{Data acquisition and pre-processing}

Continuous EEG scans were acquired using a Neuroscan EEG recording and acquisition system (SynAmps2, Compumedics, Inc.) with $64 \mathrm{Ag} / \mathrm{AgCl}$ scalp electrodes molded on an elastic cap in a 10-20 montage. Individual electrode locations were registered using the FASTRAK 3D digitizing system (Polhemus Inc.). Recordings were made against the center (near $\mathrm{Cz}$ ) reference electrode on the Neuroscan cap and computed at a sampling rate of 1,000 Hz. Channel impedances were maintained at values $<10 \mathrm{k} \Omega$. Pre-processing was performed using the EEGLAB (Delorme and Makeig, 2004) toolbox and customized MATLAB codes (version R2020a). Continuous data were high-passed at $0.1 \mathrm{~Hz}$ with finite impulse response (FIR) filter, low-passed at $80 \mathrm{~Hz}$ FIR filter, and Notch-filtered between 46 to $54 \mathrm{~Hz}$ to remove the line noise (9th order 2-pass Butterworth filter). The noisy channels were visually inspected, and the trials with large jittery amplitudes were removed, following which the data was average re-referenced. Epochs of $0.8 \mathrm{~s}(-1.2 \mathrm{~s}$ to $-0.4 \mathrm{~s})$ before the stimulus onset (prestimulus) and $0.8 \mathrm{~s}$ post the onset of the stimulus (poststimulus) were extracted using the trigger information (Figure 2). The extracted epoch data were sorted based on the Congruent AV stimuli: /pa/, /ta/, and $/ \mathrm{ka} /$ and incongruent McGurk stimulus. The sorted prestimulus epochs were then baseline corrected by removing the temporal mean of the EEG signal on an epoch-by-epoch basis. However, for the poststimulus $0.8 \mathrm{~s}$ epoch, baseline correction was done by removing the temporal mean of the EEG signal $0.2 \mathrm{~s}$ before the onset of the stimulus. Finally, to remove the response 
contamination from ocular and muscle-related artefacts, epochs with amplitudes above and/ or below $\pm 75 \mu \mathrm{V}$ were removed from all electrodes.

\subsection{Behavioral analysis}

For the incongruent McGurk stimulus, visual / $\mathrm{ka} /$ was paired with auditory / $\mathrm{pa} /$ to induce the illusory percept /ta/. Also, three congruent AV stimuli (/pa/, /ta/, and / $\mathrm{ka} /)$ with zero lag between the audio and visual input was presented to the participants. As the participants observed the four stimuli presented at random, they reported if they heard either $/ \mathrm{pa} /, / \mathrm{ta} /, / \mathrm{ka} /$, or "something else," being unaware of the McGurk illusion. Based on their McGurk susceptibility, we classified the participants into two groups: (1) rare perceivers - $<50 \% / \mathrm{ta} /$ percept, and (2) frequent perceivers - $>50 \% / \mathrm{ta} /$ percept. This classification was for subsequent analysis associated with inter-individual differences between these two specific groups. Furthermore, with these between-group classifications (rare and frequent groups), inter-trial variability was also computed for and analyzed. The inter-trial variability (or response tendency) was computed as the relative proportion of illusory $(/ \mathrm{ta} /$ ) responses in all the McGurk trials across all participants belonging to the respective perceiver group.

\subsection{Spectral analysis}

We computed the spectral power to investigate inter-subject and inter-trial differences of participants placed in either of the two groups: rare and frequent groups of perceivers based on their behavioral responses, for both the prestimulus and the poststimulus epoch data. Power spectra were computed to extract the distribution of signal power over different frequency bands (theta (4-7 Hz), alpha (8-12 $\mathrm{Hz})$, beta $(15-30 \mathrm{~Hz})$, and gamma $(31-45 \mathrm{~Hz})$ ) for all the stimulus conditions (incongruent McGurk, and congruent) using customized MATLAB scripts and the Chronux toolbox function mtspectrumc.m 
(Bokil et al., 2010). Time bandwidth product and the number of tapers used were set to 3 and 5 , respectively.

\subsection{Extracting the periodic and aperiodic components from the power spectrum}

The power spectrum has rhythmic oscillations manifested as narrowband peaks over the aperiodic (or non-oscillatory) component. So, parameterizing the power spectrum is required. The aperiodic component of a power signal can be characterized in terms of slope (or exponent) (Gao et al., 2017; Freeman \& Zhai, 2009), which has been correlated with the integration of the underlying synaptic currents (Buzsáki et al., 2012). And offset of the broadband power of the signal, which is associated with neuronal population spiking (Manning et al., 2009; Miller et al., 2012) and the BOLD (bloodoxygen-level-dependent) signal of fMRI (Winawer et al., 2013).

To separate the "background" 1/f aperiodic component from its periodic counterpart, we used FOOOF (or Fitting Oscillations and One Over $f$ ) algorithm developed by Donoghue et al., 2020. FOOOF algorithm takes the original power spectral densities (PSDs) and extracts the aperiodic signal, and superimposes them on periodic oscillatory components, referred to as "peaks." These peaks are considered to be oscillations and are modelled individually as Gaussian functions. Each of these Gaussian has three parameters (also see Figure 1) that are used to define an oscillation. The formula for fitting the power spectrum is as follows:

$$
P=L+\sum_{n=0}^{N} G_{n}
$$


Where $P$ is power, it is a linear combination of the aperiodic signal $L$ with the $N$ total number of Gaussians $G$. Each $G_{n}$ is a Gaussian fit to the peak for $N$ total number of peaks extracted from the power spectrum, modelled as:

$$
G_{n}=a * \exp \left(\frac{-(F-c)^{2}}{2 w^{2}}\right)
$$

Where $a$ is the amplitude, $c$ is the center frequency, $w$ is the bandwidth of the Gaussian, and $F$ is the vector of input frequencies. Furthermore, the aperiodic signal $L$ is modelled using an exponential function in semi log-power space (linear frequencies and logged power values) as the following:

$$
L=b-\log \left(k+F^{\chi}\right)
$$

Where $b$ is the aperiodic broadband offset, $\chi$ is the aperiodic slope, and $k$ is the "knee" parameter, controlling for the bend in the aperiodic signal, with $F$ as the vector of input frequencies. The above equation is equivalent to fitting a line in $\log$ - $\log$ space, when $k=0$, referred to as the "fixed" model. The final output of the algorithm is the parameters defining the best model fit for the aperiodic signal and the $N$ Gaussians. Along with the Gaussian parameters, transformed parameters are also returned. These parameters involve (1) center frequency which is the mean of the Gaussian; (2) amplitude of the peak, which is the distance between the peak of the Gaussian and the aperiodic fit, and (3) bandwidth as two standard deviations.

The periodic and the aperiodic components of the spectral data were extracted for the two epoch conditions (prestimulus $0.8 \mathrm{~s}$ and poststimulus $0.8 \mathrm{~s}$ ) using the FOOOF algorithm. The algorithm is an open-source Python Package with package dependencies limited to NumPy, matplotlib, and scipy ( $\geq$ 
version 0.19). The FOOOF algorithm (version 1.0.0) was used to parameterize neural power spectra. Power spectra were parameterized across the frequency range 0.1 to $45 \mathrm{~Hz}$ using customized Python scripts. FOOOF was performed to the inter-individual and inter-trial spectral data of the two groups (rare and frequent) for all the stimulus conditions (incongruent and congruent). The periodic oscillations were extracted by subtracting the aperiodic fit from original PSDs. Subsequently, the differences in the periodic oscillations and aperiodic features (aperiodic fit) between the two groups for all the different frequency bands were statistically compared using permutation tests at 1000 iterations followed by a two-tailed t-test. Finally, we also applied the Wilcoxon rank-sum test (nonparametric) and two-tailed t-test (parametric) to statistically compare periodic components (center frequency, adjusted power, and bandwidth) and aperiodic components (offset and exponent) between the two groups of perceivers at inter-individual and inter-trial level respectively, after checking the normality of the data distribution by using Kolmogorov Smirnov test.

\subsection{Large-scale functional connectivity analysis at the sensor level}

Global coherence was computed to investigate frequency-specific functional connectivity (FC) that subserves the cross-modal speech perception and to characterize inter-subject variability differences and inter-trial variability between the rare and frequent group of perceivers (Cimenser et al., 2011, Kumar et al., 2020). It is a multivariate spectral analysis technique used to capture and quantify the strength of the covariation of neural oscillations at the global (or whole brain) scale (Mitra, P. 2007). We employed the Chronux function CrossSpecMatc.m to obtain trial-wise global coherence for the prestimulus and poststimulus epoch data, which were already sorted based on stimuli and responses. Three orthogonal discrete prolate spheroidal sequences (or dpss) were considered. The dpss, also known as Slepian tapers, is used to reduce leakage of the spectral estimates into the nearby frequency 
bins. The time-bandwidth chosen was five that resulted in a frequency bandwidth of $0.25 \mathrm{~Hz}$. The output variable "Ctot" of the function yielded the total global coherence value at frequency $f$. The function multiplies the given epoch data with the specified number of Slepian tapers and then performs the fast Fourier transform $(F F T)$. The resulting FFT values are then averaged, and the crossspectrum for all the sensor combinations at frequency $f$ is computed. The cross-spectral density between any two sensors is then computed from the tapered Fourier transforms using the following equation:

$$
C_{i j}=\operatorname{conj}\left(X_{i}(f) \cdot X_{j}(f)\right)
$$

Where $C_{i j}$ represents the cross-spectrum, $X_{i}$ and $X_{j}$ represent the tapered Fourier transforms from the sensors $i$ and $j$, respectively. Subsequently, singular value decomposition (SVD) was applied on the cross-spectral density matrix for the specified frequency $f$, which yields the following:

$$
D(f)=V C V^{T}
$$

The diagonal matrix $D$ comprises the values corresponding to the variance explained by the orthogonal set of eigenvectors $\left(V, V^{T}\right)$. Finally, the global coherence $C_{\text {Global }}(f)$ at frequency bin $f$ was computed by normalizing the largest eigenvalue (or the first value of the $D(f)$ at frequency $f$ ) on a trial-by-trial basis for each participant in the two groups, employing the following equation:

$$
C_{\text {Global }}(f)=\frac{D_{1}(f)}{\sum_{i=1}^{n} D_{i}(f)}
$$

To capture the inter-subject and inter-trial variability, the coherences (or Ctot values) of the two groups for all the stimulus conditions (incongruent and congruent) were compared for the significant 
differences at different frequency bands (theta, alpha, beta, and gamma) explicitly by employing cluster-based permutation test (see section 2.8 for further details).

\subsection{Statistical analysis for the sensor-level global coherence}

The statistical test used for the global coherence (section 2.7) was the cluster-based permutation testing (Maris, Schoffelen, and Fries 2007). For every frequency bin at each time-point, the corresponding differences (coherence values) between the two groups: the rare and the frequent perceivers were evaluated using the Fisher's Z transformation:

$$
Z(f)=\frac{\tanh ^{-1}\left(C_{1}(f)\right)-\tanh ^{-1}\left(C_{2}(f)\right)-\left(\left(\frac{1}{2 m_{1}-2}\right)-\left(\frac{1}{2 m_{2}-2}\right)\right)}{\sqrt{\frac{1}{2 m_{1}-2}+\frac{1}{2 m_{2}-2}}}
$$

Where $2 m_{1}, 2 m_{2}=$ degrees of freedom in the first and second condition; $Z(f) \approx N(0,1)$ a unit normal distribution; and $C_{1}$ and $C_{2}$ are the corresponding differences (of coherence) at frequency $f$. The Zstatistic matrix obtained from the above computation formed the observed Z-statistics. Subsequently, clusters from the observed Z-statistics matrix were selected based on oscillatory frequencies (4-7 Hz, theta; 8-12 Hz, alpha; $15-30 \mathrm{~Hz}$, beta; $31-45 \mathrm{~Hz}$, gamma). Following the computation of the clusterlevel statistics of the observed Z-statistics, 1000 iterations of trial randomization were carried out in the case of global coherence data. A cluster-level statistic was computed for every iteration of the randomized trials to generate the permutation distribution. Subsequently, the values of the observed cluster-level statistics were compared with the $5^{\text {th }}$ and $95^{\text {th }}$ quantile values of the respective permutation distribution. The observed cluster-level statistics value that was above the $95^{\text {th }}$ and below the $5^{\text {th }}$ quantile subsequently for two time-points formed the positive and negative clusters, respectively. 


\subsection{Source reconstruction using Colin-27 template}

We employed dynamic imaging of coherent sources to determine the sources for the rare and frequent group of perceivers in both prestimulus and poststimulus duration (DICS; Gross J. et al., 2001). DICS beamformer extends the spatial filter algorithm to the frequency domain. The spatial filter matrix for frequency $f$ is estimated by:

$$
W_{s}(f)=\frac{L^{T} C^{-1}(f)}{L^{T} C^{-1}(f) L}
$$

Where $C(f)$ is the cross-spectral density matrix for the frequency $f$, and $L$ is the leadfield matrix. Customized MATLAB codes employing the FieldTrip toolbox were used (Oostenveld R. et al., 2011). The leadfield matrix for the Colin 27 brain template was generated using $f t$ prepare_leadfield. $m$ and employing the boundary element method (BEM). Then, we computed individual participants' crossspectral density (CSD) matrices across all incongruent and congruent trials for the prestimulus and poststimulus epochs using ft freqanalysis.m. Both leadfield matrix and CSD matrices were used to estimate the common spatial filters. Common filters estimate the difference in the source power activity across the two trial conditions, independent of the differences in the filter output (that may arise due to variations in the signal-to-noise ratio and eventually varying CSD matrices). Using this common inverse filter, the power of the underlying sources was estimated for each participant. The statistical threshold was set to the $95^{\text {th }}$ percentile, and the source power that crossed this threshold was considered a significant source of activation. 


\subsection{Source time-series reconstruction and coherence analysis}

The statistically significant source power coordinates for both the prestimulus and poststimulus durations were used to reconstruct the time series for each participant. This was achieved by multiplying the spatial filter generated using the computed source grid points with the processed EEG time-series data. We used common electrode placements across all participants, which were computed by taking the average of each participant's Polhemus 3D locations with the origin set to Cz. By multiplying the filter with the EEG data, 3 source dipole time-series with their orientations along the $\mathrm{x}, \mathrm{y}$, and $\mathrm{z}$-axis directions were obtained. For interpreting the results, however, we projected the time-series along the strongest dipole direction. This was achieved by estimating the largest (temporal) eigenvector corresponding to the first singular value. Furthermore, using k-means clustering, the source grid points were classified into nodes based on the centroid of each cluster. Sources were then classified into 10 and 8 nodes during prestimulus and 10 and 9 nodes in poststimulus durations, respectively, for rare and frequent groups. Finally, each participant's reconstructed time-series was considered a single trial, making a total of 8 trials for the rare group and 10 trials for the frequent group. The time-series corresponding to each node was reconstructed for $800 \mathrm{~ms}$ duration for both the prestimulus and poststimulus duration. Finally, to compute the functional connectivity strength between these sources, we calculated the imaginary part of the coherence (Nolte et al., 2004), the reason being that the imaginary part of the coherence captures the true source interactions at a given time lag by eliminating all the spurious source interactions that arise due to volume conduction, cross talk, and other instantaneous activity in EEG. The coherence between the source node time-series was computed using the Chronux function CrossSpecMatc.m. to obtain a coherence matrix. Subsequently, we extracted the imaginary part of the coherence matrix to look at the connectivity strength between the corresponding sources 
during the prestimulus and poststimulus duration. We visualized the coherence strength using BrainNet Viewer (Xia et al., 2013).

\section{Results}

\subsection{Substantial variability in illusory percept captured within and between participants.}

Participants were subjected to four kinds of AV stimuli: incongruent McGurk (visual $/ \mathrm{ka} / \mathrm{paired}$ with auditory /pa/ to induce the illusory percept /ta/) and congruent (video and audio synched) /pa/, /ta/, $/ \mathrm{ka} /$, presented at random. Participants were instructed to report whether they perceived $/ \mathrm{pa} /, / \mathrm{ta} /, / \mathrm{ka} /$, or "something else.", unaware of the McGurk illusion. Previously we have shown a high degree of inter-individual variability among the participants perceiving the McGurk illusion (Kumar et al., 2020). Based on their McGurk susceptibility, the participants were classified into two groups, rare group of perceivers $(<50 \% / t a /$ percept $)$ and frequent group of perceivers $(>50 \% / t a /$ percept $)$. This classification was for subsequent analysis associated with inter-individual variability between these two specific groups. Eight of the eighteen participants showed $<50 \%$ susceptibility towards the McGurk effect. The remaining number of participants showed $>50 \%$ susceptibility towards the McGurk illusion (Figure 3a). Furthermore, the inter-trial variability (or response tendency) was computed as the relative proportion of responses for respective stimuli (congruent and incongruent) in all the trials across all participants belonging to the respective perceiver group (Bechtold \& Bastian, 2016; Figure 3b). It was found that rare group participants on average reported an illusory /ta/percept $26.95 \%(\mathrm{SD}=17.62)$ of trials, whereas a unisensory $/ p a /$ percept was reported in $69.35 \%(\mathrm{SD}=$ 15.64) of trials, for the incongruent McGurk stimulus condition. Contrastingly, on average, the frequent group participants reported an illusory $/ \mathrm{ta} /$ percept $83.52 \%(\mathrm{SD}=13.18)$ of trials, and a 
unisensory $/ p a /$ percept was reported in $11.82 \%(\mathrm{SD}=10.14)$ of trials for the incongruent McGurk stimulus. Congruent AV stimuli (/pa/, /ta/, and $/ \mathrm{ka} /)$, in the case of rare group, were correctly identified in $95.72 \%(\mathrm{SD}=3.07)$ of trials. Whereas for the frequent group, the congruent AV stimuli were reported in $97.19 \%(\mathrm{SD}=2.09)$ of the trials. The difference between the percentage of $/ \mathrm{ta} /$ and $/ p a /$ percept during the McGurk stimulus was significant in both the rare $\left(t_{14}=-5.08, p<0.001\right)$ and frequent $\left(t_{18}=13.63, p<0.001\right)$ group of perceivers.

\subsection{Periodic and aperiodic components of oscillatory activity}

To separate the aperiodic component (or background $1 / f$ component) from the periodic component in both the prestimulus $0.8 \mathrm{~s}$ and poststimulus $0.8 \mathrm{~s}$ epoch data of the rare and frequent group of perceivers, FOOOF algorithm was performed. Power spectra capturing inter-individual variability and inter-trial variability were parameterized across the frequency range 0.1 to $45 \mathrm{~Hz}$. Subsequently, the interindividual differences in the periodic oscillations (original PSDs - aperiodic fit) and aperiodic component (aperiodic fit) between the two groups for all the different frequency bands were statistically compared using permutation tests at 1000 iterations followed by a two-tailed t-test. Wilcoxon rank-sum test was applied to calculate significant differences between periodic parameters (center frequency, adjusted power, and bandwidth) and aperiodic parameters of the two groups (offset, exponent). For inter-trial variability, however, we compared the periodic oscillations and aperiodic components using the permutation test and the parameters using a two-tailed t-test.

\subsubsection{Periodic and Aperiodic components of inter-individual variability.}

In the prestimulus duration, it was observed that before McGurk stimulus onset, the periodic oscillations of the rare group showed a significant increase in both the alpha $(t=4.801, p=0.007)$ 
and beta $(t=5.279, p<0.0001)$ bands than the frequent perceivers (Figure 4a). This difference in alpha periodic power was concentrated maximum over the frontal, fronto-central and temporal sensors on the left; and parieto-occipital and occipital sensors on the right. Whereas the difference in the beta band was maximum over the frontal, fronto-central and temporal sensors on the left; and centro-parietal and occipital sensors on the right (Figure 4a, topoplots). Similarly, for congruent stimuli, we observed a significant increase in alpha band (Cong /pa/: $t=8.648, p=0.001$; Cong $/$ ta $/$ : $t=5.746, p=0.002 ;$ Cong $/ \mathrm{ka} /: t=7.879, p<0.0001)$ and beta $($ Cong $/ p a /: t=5.903, p<0.0001$; Cong $/ \mathrm{ta} /: \mathrm{t}=6.464, \mathrm{p}<0.0001 ;$ Cong $/ \mathrm{ka} /: t=6.216, p<0.0001$ ) bands (Supplementary Figure 1a). The difference of alpha power was observed at maximum in the temporo-parietal sensor on the right, and anterior-frontal, fronto-temporal, centro-parietal sensors on the left for the congruent /pa/ and $/ \mathrm{ta} /$ stimulus conditions. However, for the congruent $/ \mathrm{ka} /$ condition, the posterior-occipital sensor PO10 on the right and posterior-occipital sensor PO9 on the left showed maximum alpha power. Only the right occipital sensor O2 showed a higher power for the beta power (Supplementary Figure 1a, topoplots).

In the case of the poststimulus epoch data's periodic component, we observed that for all the stimulus conditions, the rare group of perceivers was characterized by a significant increase in the alpha $($ McGurk: $t=3.888, p=0.025 ;$ Cong $/ p a /: t=3.986, p=0.021 ;$ Cong $/ t a /: t=3.237, p=0.04$; Cong $/ k a /: t=3.170, p=0.042)$ and beta (McGurk: $t=7.270, p<0.0001 ;$ Cong $/ p a /: t=6.924, p<$ $0.0001 ;$ Cong /ta/: $t=7.221, p<0.0001 ;$ Cong $/ \mathrm{ka} /: t=8.705, p<0.0001)$ bands, followed by a significant decrease in the gamma band (McGurk: $t=-7.734, p<0.0001$; Cong /pa/: $t=-6.223, p<$ 0.0001; Cong $/ \mathrm{ta} /: t=-6.846, p<0.0001 ;$ Cong $/ \mathrm{ka} /: t=-6.863, p<0.0001$ ) (Figure 4a, Supplementary Figure 1b). The difference in the alpha band was maximum at the fronto-parietal, 
anterior-frontal, fronto-central, central, fronto-temporal, centro-parietal, and temporal sensors on the left; and anterior-frontal, fronto-parietal, temporo-parietal, parieto-occipital, and occipital sensors on the right for both the incongruent and congruent stimulus conditions. The exception is for the congruent $/ \mathrm{ka}$ / condition, where only the parieto-occipital PO10 and occipital O2 sensors on the right and parieto-occipital PO9 sensor on the left showed the maximum difference. Similarly, for the beta power frontal, fronto-central, fronto-temporal, temporal, and occipital sensors on the left; and frontal, temporal, temporo-parietal, occipital on the right was maximum for incongruent and congruent $/ \mathrm{pa} /$ and $/ \mathrm{ta} /$ stimuli. For congruent $/ \mathrm{ka} /$, sensor F1 on the left and AF4, F8, P8, and $\mathrm{O} 2$ sensors on the right showed the maximum difference in beta power. And, finally, the difference in gamma band anteriorfrontal, frontal, fronto-central, central, centro-parietal, and parieto-occipital on the left and also on the right was minimum for incongruent and congruent stimulus conditions, except in congruent $/ \mathrm{ka} /$ condition where PO9 sensor on the left and PO10 sensor on the right showed minimum gamma-band activity for the rare group as compared to the frequent group (Figure 4a, Supplementary Figure 1b, topoplots).

Furthermore, the aperiodic component of the prestimulus epochs was also statistically analyzed. We observed that before the incongruent and congruent stimuli, the rare group elicited significantly lower beta band (McGurk: $t=-3.829, p=0.00068$; Cong /pa/: $t=-3.015, p=0.0055$; Cong $/$ ta $/: t=-$ 2.903, $p=0.0072$; Cong $/ \mathrm{ka} /: t=-3.001, p=0.0057)$ and lower gamma band activity (McGurk: $t=$ -8.726, $p<0.0001 ;$ Cong $/ \mathrm{pa} /: t=-7.416, p<0.0001 ;$ Cong $/ \mathrm{ta} /: t=-7.035, \mathrm{p}<0.0001$; Cong $/ \mathrm{ka} /$ : $t=-7.181, p<0.0001$ ) than the frequent group (Figure 4b, Supplementary Figure 2a). Moreover, only the offset aperiodic parametric distribution between the two groups differed significantly (MannWhitney $U=60, p=0.041$, one-sided) (seaborn violin plot - Waskom M.L. 2021; Figure 5). The 
sensor-wise differences of the beta and gamma power were seen for both incongruent and congruent stimulus conditions were maximum over the central, centro-parietal, temporal, and frontal sensors on the right; and occipital and parieto-occipital sensors on the left. The exception is during the congruent $/ k a /$ condition, where differences in both the beta and gamma power were maximally distributed across all brain regions on almost all left and right-side sensors except for FT8, PO10, and O2 sensors on the right and PO9 sensor on the left (Figure 4b, Supplementary Figure 2a, topoplots).

Finally, in the case of post-stimulus epochs, we observed that for McGurk stimulus' and congruent /pa/ stimulus' aperiodic distribution, the rare group elicited a lower beta band (McGurk: $t=-2.374$, $p=0.025 ;$ Cong $/ p a /: t=-2.047, p=0.0502)$ and a lower gamma-band activity $($ McGurk: $\mathrm{t}=-5.477$, $p<0.0001 ;$ Cong $/ p a /: t=-4.896, p<0.0001$ ) than the frequent perceivers (Figure 4b, Supplementary Figure 2b), whereas for congruent $/ t a /$ and $/ k a /$ stimuli, rare group showed a significant lower gamma-band activity only (Cong /ta/: $t=-4.019, p=0.00045$; Cong $/ \mathrm{ka} /: t=-$ 3.947, $p=0.00054$ ) (Supplementary Figure 2b). During the McGurk and congruent / $\mathrm{pa} /$ condition, the frontal, central, centro-parietal, and temporal sensors on the right showed the maximum difference in the beta-band aperiodic component. Also, the sensor-wise difference in the gamma band activity was maximum at frontal, central, and temporal sensors on the right during both incongruent and congruent stimuli. The exception was for congruent $/ \mathrm{ka} /$ stimulus, where a higher gamma-band difference was observed across all the sensors except for FT8, O2, PO10 sensors on the right, where the difference was at a minimum (Figure 4b, Supplementary Figure 2b, topoplots). 


\subsubsection{Periodic and Aperiodic components of inter-trial variability.}

The periodic and aperiodic component was extracted from trials that were sorted based on the perceptual categories (McGurk: /ta/- illusory and/pa/- unisensory, congruent: /pa/, /ta/, and /ka/) over all the participants categorized into two groups (rare and frequent) and were statistically compared using two-tailed $t$-test. For the pre-stimulus epoch data, we observed that the periodic oscillations of the frequent perceivers were characterized by a significant increase in the alpha (for McGurk/ta/: $t$ $=11.464, p<0.0001 ;$ Cong $/ p a /: t=5.383, p=0.00099$ ), and beta (for McGurk $/ p a /: t=6.998, p<$ 0.0001; McGurk/ta/: $t=2.606, p=0.0146 ;$ Cong $/ p a /: t=6.486, p<0.0001 ;$ Cong $/ t a /: t=5.829$, $p<0.0001$; Cong $/ k a /: t=3.913, p=0.0006$ ) bands (Figure 6a, Supplementary Figure 3a). The difference in the periodic beta power was maximum at the central sensors and centro-parietal sensors on the right for all the stimulus-response conditions except for unisensory McGurk / $\mathrm{pa} /$ response, where the difference was maximum at the frontal, fronto-central, and central sensors on the left (Figure 6a, Supplementary Figure 3a, topoplots). For the post-stimulus epochs, the periodic component of the frequent perceivers showed significant increase in the alpha band during the McGurk /ta/ (illusory) $(t=12.673, p<0.0001)$, congruent $/ p a /$ response $(t=7.298, p=0.00062)$, and congruent $/ \mathrm{ka} /(t=5.145, \mathrm{p}=0.0027)$, significant increase in the beta band during unisensory McGurk $/ p a /(t=7.489, p<0.0001)$, and significant decrease in the gamma band during the congruent $/ p a /(t=-2.800, p=0.0112)$ and congruent $/ t a /(t=-3.050, p=0.0065)($ Figure 6a, Supplementary Figure 3b). The difference for the periodic alpha power was maximum at the central and centro-parietal sensors on the right for illusory $/ \mathrm{ta} /$, congruent $/ \mathrm{pa} /$, and congruent $/ \mathrm{ka} /$ conditions. For unisensory McGurk/pa/response, the periodic beta power difference was maximum at the frontal, central, centro-parietal, and parietal sensors on the right; and frontal, fronto-parietal, central, centroparietal, parietal, parieto-occipital, and occipital sensors on the left. Finally, the difference in the 
gamma power was maximum FT8, FT7, PO10, and PO9 sensors for congruent /pa/ and congruent /ta/ conditions (Figure 6a, Supplementary Figure 3b, topoplots).

Interestingly enough, the pre-stimulus aperiodic component of the rare group of perceivers for all the response tendencies elicited higher $1 / f$ distribution in the beta-band (McGurk $/ p a /: t=3.198, p=$ 0.0034; McGurk/ta/: $t=5.035, p<0.0001 ;$ Cong $/ p a /: t=4.387, p=0.00015 ;$ Cong $/$ ta $/: t=4.445$, $p=0.00012$; Cong $/ k a /: t=2.894, p=0.0073)$ and gamma frequency bands $($ McGurk $/ p a /: t=6.199$, $p<0.0001 ;$ McGurk /ta/: $t=9.067, p<0.0001 ;$ Cong /pa/: $t=8.448, p<0.0001$; Cong $/$ ta $/: t=$ 8.022, $p<0.0001 ;$ Cong $/ k a /: t=5.730, p<0.0001)$. Also, we observed a higher alpha-band activity in rare group during the McGurk illusory $/ \mathrm{ta} /(t=4.046, p=0.0067)$, and the congruent $/ \mathrm{ta} /(t=$ 3.560, $p=0.012$ ) (Figure 6b, Supplementary Figure 4a). Moreover, for the post-stimulus duration we obtained a higher beta band activity for the rare perceivers during McGurk illusory /ta/ percept $(t$ $=2.167, p=0.0389)$. And, higher gamma band activity was observed during McGurk/ta/ illusory $(t$ $=3.548, p=0.00149)$, and the three congruent conditions $($ Cong $/ p a /: t=3.527, p=0.0016$; Cong $/ t a /: t=2.760, p=0.0104 ;$ Cong $/ k a /: t=2.775, p=0.010)($ Figure 6b, Supplementary Figure 4b). The difference in alpha, beta, and/or gamma band aperiodic component for all stimulus conditions during prestimulus and poststimulus duration was maximum at the frontal, central, and centro-parietal sensors on the right. Except during prestimulus unisensory McGurk/pa/ condition, where the difference in the beta and gamma band aperiodic component was maximum at F7, C5, and $\mathrm{O} 1$ sensors on the left; and F6, F8, FC6, C6, and C8 sensors on the right (Figure 6b, Supplementary Figure 4b, topoplots). 
Finally, the periodic parameters (center frequency CF, adjusted power PW, and bandwidth BW) and aperiodic parameters (exponent, offset) differed significantly between the two-groups, both during pre-stimulus (offset: $t=7.44, p<0.0001$; CF: $t=-2.70, p=0.0069$; PW: $t=-5.224, p<0.0001$,; BW: $t=-7.02, p<0.0001$ ); and post-stimulus (exponent: $t=3.34, p=0.0008$; offset: $t=10.387, p$ $<<0.0001$; PW: $\mathrm{t}=-16.83, p<<0.0001$; BW: $t=-7.48, p<<0.0001$ ) duration differed significantly

\section{(Supplementary Figure 5).}

\subsection{Frequency specific large-scale functional connectivity}

In the previous sections we report the inter-individual and inter-trial variability in parametrized power (periodic oscillations and aperiodic component) between the rare and frequent group of the McGurk perceivers. However, since complex cognitive traits arise due to the interactions among large-scale neuronal networks (Varela et al., 2001; Bressler \& Menon, 2010), we hypothesized that the two groups of perceivers might also have different functional connectivity dynamics that might hint on the variability in McGurk perception. Therefore, to capture these neural signatures we used global coherence (or $C_{\text {tot }}$ values) at different frequency bands (theta: 4-7 Hz; alpha: 8-12 Hz; beta: 15-30 Hz, and; gamma: $31-45 \mathrm{~Hz}$ frequency bands). We computed the time-average global coherence on the prestimulus and poststimulus epochs for incongruent and congruent stimulus conditions.

\subsubsection{Frequency specific connectivity index of inter-subject variability.}

During the pre-stimulus duration, we observed that before the incongruent and congruent stimulus conditions the rare group had a significantly higher theta coherence (McGurk: $z_{95}=0.42$; Cong $/ p a /$ : $z_{95}=0.49 ;$ Cong $/ \mathrm{ta} /: z_{95}=0.48 ;$ Cong $\left./ \mathrm{ka} /: z_{95}=0.44\right)$, higher alpha coherence $\left(\right.$ McGurk: $z_{95}=0.44 ;$ Cong /pa/: $z_{95}=0.39 ;$ Cong /ta/: $z_{95}=0.78 ;$ Cong $/ \mathrm{ka} /: z_{95}=0.76$, followed by a lower gamma 
coherence (McGurk: $z_{5}=-0.47 ;$ Cong /pa/: $z_{5}=-0.56$; Cong $/ \mathrm{ta} /: z_{5}=-0.44 ;$ Cong $/ \mathrm{ka} /: z_{5}=-0.68$ ) than frequent group of perceivers. We also observed a higher beta coherence for the rare group before incongruent $\left(z_{95}=0.34\right)$, congruent $/ p a /\left(z_{95}=0.20\right)$, congruent $/ t a /\left(z_{95}=0.32\right)$ stimulus presentation (Figure 7a, Supplementary Figure 6a, Supplementary Table 1a). Furthermore, for the poststimulus epoch data for all the stimulus conditions were computed and analysed. We observed that the rare perceivers showcased an enhanced global coherence in the theta (McGurk: $z 95=0.26$; Cong $/ p a /: z_{95}=0.31 ;$ Cong $/ \mathrm{ta} /: z_{95}=0.50 ;$ Cong $\left./ \mathrm{ka} /: z_{95}=0.34\right)$, alpha $\left(\right.$ McGurk: $z_{95}=1.00 ;$ Cong $/ p a /$ : $z_{95}=0.79 ;$ Cong /ta/: $z_{95}=0.66 ;$ Cong /ka/: $\left.z_{95}=1.02\right)$, and beta (McGurk: $z_{95}=0.56 ;$ Cong /pa/: $z_{95}=0.32 ;$ Cong $/ \mathrm{ta} /: z_{95}=0.42 ;$ Cong $/ \mathrm{ka} /: z_{95}=0.28$ ); followed by a lower gamma coherence (McGurk: $z_{5}=-0.40 ;$ Cong $/ p a /: z_{5}=-0.62 ;$ Cong $/ \mathrm{ta} /: z_{5}=-0.47 ;$ Cong $\left./ \mathrm{ka} /: z_{5}=-0.43\right)($ Figure 7a,

\section{Supplementary Figure 6b, Supplementary Table 1b).}

\subsubsection{Frequency specific interactions index of inter-trial variability.}

The time-averaged global coherence was computed on trials based on the perceptual categories (/ta/ and $/ p a /$ ) over the participants categorized into the two groups (rare and frequent) and were statistically compared by means of the cluster-based permutation test. For the pre-stimulus duration, we observed that rare group elicited a higher beta band coherence (McGurk /ta/: z95 =0.87; McGurk $/ p a /: z_{95}=0.98 ;$ Cong /pa/: $z_{95}=0.89 ;$ Cong /ta/: $z_{95}=0.52 ;$ Cong $\left./ \mathrm{ka} /: z_{95}=0.97\right)$ and gamma band coherence (McGurk/ta/: $z_{95}=0.96 ;$ McGurk/pa/: $z_{95}=0.76 ;$ Cong /pa/: $z_{95}=0.95 ;$ Cong /ta/: $z_{95}=$ 0.55; Cong $/ \mathrm{ka} /: z_{95}=0.95$ ) activity for both incongruent (illusory and unisensory) and congruent stimulus conditions (Figure 7b, Supplementary Figure 7a, Supplementary Table 2a). Finally, in the case of post-stimulus epoch data, we observed that for the illusory /ta/ percept, the rare group elicited a higher alpha coherence $\left(z_{95}=0.61\right)$ and beta band coherence $\left(z_{95}=0.57\right)$ activity. In case 
of non-illusory McGurk response, the rare group's coherence was higher at all frequency ranges (theta: $z_{95}=0.62 ;$ alpha: $z_{95}=0.63 ;$ beta: $z_{95}=0.68 ;$ gamma: $z_{95}=0.80$ ) than the frequent group. In congruent stimulus state the rare group had a higher $/ \mathrm{pa} /$ alpha $\left(z_{95}=0.87\right), / \mathrm{pa} /$ beta $\left(z_{95}=0.51\right)$; $/ \mathrm{ta} /$ beta $\left(z_{95}=0.42\right), / \mathrm{ta} / \mathrm{gamma}\left(z_{95}=0.54\right) ;$ and $/ \mathrm{ka} /$ theta $\left(z_{95}=0.58\right), / \mathrm{ka} /$ alpha $(z 95=0.47), / \mathrm{ka} /$ beta $\left(z_{95}=0.46\right)($ Figure 7b, Supplementary Figure 7b, Supplementary Table 2b).

\subsection{Underlying cortical sources of neural activity during prestimulus duration.}

We employed source estimation to identify the cortical sources that were responsible for the prestimulus state functional dynamics. The underlying sources in the rare group's incongruent (McGurk) condition with respect to the congruent condition were calculated using the DICS beamformer (see section 2.9) and were compared with the sources of the frequent group of perceivers computed similarly. The source power of all participants in the rare group and the frequent group were grand-averaged and tested for statistical significance. The sources that crossed the $99.5^{\text {th }}$ percentile threshold were considered significant sources. The cortical regions that showed significant activations are listed in table 1 and supplementary table 2 . The underlying sources for the rare group of perceivers were left and right anterior prefrontal cortex, left and right Broca's opercularis (or inferior frontal gyrus), right dorsolateral prefrontal cortex (both dorsal and lateral), right superior temporal gyrus (part of Wernicke's area), right angular gyrus, right visual-motor area, and left premotor and supplementary motor cortex. Similarly, for the frequent group of perceivers, the sources were left and right anterior prefrontal cortex, left and right Broca's opercularis (or inferior frontal gyrus), right superior temporal gyrus, right angular gyrus, right visual-motor area, and left premotor and supplementary motor cortex. The cortical sources responsible during the poststimulus duration for the rare group were left and right anterior prefrontal cortex, right Broca's opercularis, left Broca's 
triangularis, right dorsolateral prefrontal cortex (both lateral and dorsal), right superior temporal gyrus, right angular gyrus, right visual-motor area, and left premotor and supplementary motor cortex. Likewise, for the frequent group of perceivers, the sources were left and right anterior prefrontal cortex, right Broca's opercularis, left Broca's triangularis, right superior temporal gyrus, right angular gyrus, right visual-motor cortex, right dorsolateral prefrontal cortex (lateral), and left the premotor and supplementary motor area.

\subsection{The coherence strength between sources for the two groups of perceivers}

The time-series reconstructed for the sources involved before and after the McGurk illusory percept were computed to estimate the functional connectivity strength between the source nodes. This was achieved by estimating the imaginary part of the coherence. We looked into the coherence strength of those corresponding frequency bands, which showed a significant difference in total coherence between the rare and frequent group of perceivers during the McGurk stimulus condition at the sensor level. The rare group showed a higher significant difference in the theta, alpha, and beta band coherence but a lower significant difference in the gamma coherence from the frequent group in the prestimulus duration. For the functional connectivity at the source-level, we observed that for rare group of perceivers during the prestimulus duration, theta coherence strength between right angular gyrus and right Broca's opercularis; right angular gyrus and left Broca's opercularis; and left Broca's opercularis and right STG was maximum (Figure 8a). For the frequent perceivers, however, the theta coherence was maximum only between the right angular gyrus and left premotor area; and right angular gyrus and right Broca's opercularis (Figure 8b). The alpha coherence was maximum between left Broca's opercularis and right visual-motor area; and right visual-motor area and right dorsolateral PFC (dorsal) (Figure 8c) and for frequent perceivers, the alpha coherence was maximum between 
left premotor area and right angular gyrus; and right angular gyrus and right Broca's opercularis (Figure 8d). The beta coherence between right angular gyrus and right dorsolateral prefrontal cortex (dorsal); right angular gyrus and right Broca's opercularis; right dorsolateral prefrontal cortex (dorsal) and right superior temporal gyrus (rSTG); and rSTG and right Broca's opercularis was maximum for the rare group (Figure 8e). For the frequent group, beta coherence between left premotor area and left Broca's opercularis; and left Broca's opercularis and right Broca's opercularis was maximum (Figure 8f). And, finally, the rare group's gamma coherence was maximum between left premotor area and right dorsolateral PFC (dorsal) (Figure 8g). For frequent perceivers, gamma coherence was seen at maximum between right Broca's opercularis and right visual-motor area (Figure 8h).

In the poststimulus duration, at the sensor level, we observed that rare perceivers showed a higher global coherence at the theta, alpha, and beta bands, followed by a lower gamma coherence than the frequent perceivers. At the source level, theta coherence was maximum between right STG and right dorsolateral PFC; and right STG and left premotor cortex for the rare group. For the frequent perceivers, right STG and left anterior PFC (Supplementary Figure 8a, 8b). Alpha coherence was maximum between right visual-motor cortex and right angular gyrus; and right dorsolateral prefrontal cortex (dorsal) and right STG for the rare group. For the frequent group, alpha coherence was maximum between left premotor area and left anterior PFC (Supplementary Figure 8c, 8d). The beta coherence of the rare group of perceivers was maximum between right visual motor and right STG; and right STG and right dorsolateral PFC (dorsal). For the frequent group, beta coherence was maximum between right visual motor and right anterior PFC (Supplementary Figure 8e, 8f). And, gamma coherence was maximum for the rare group between right visual-motor area and right STG. For the frequent group, gamma coherence was maximum between right angular gyrus and right STG; 
right angular gyrus and right Broca's opercularis; and right visual motor and right STG (Supplementary Figure 8g, 8h).

\section{Discussion}

In this study, we analyzed the prestimulus EEG data of the participants that were subjected to the incongruent McGurk and the congruent $/ \mathrm{pa} / \mathrm{/ta} /$, and $/ \mathrm{ka} /$ stimuli. Based on the overall percentage of illusory $/ \mathrm{ta} /$ percept response, the participants were placed in either a rarely $(<50 \%)$ or a frequently ( $>50 \%$ ) perceiving group of McGurk illusion. We were interested in capturing differences in the brain activity of the two groups of perceivers at an inter-individual and inter-trial level during the prestimulus duration. In other words, we were interested in looking for specific neural signatures in the prestimulus duration that can differentiate a rare perceiver from a frequent perceiver of McGurk illusion by looking at their inter-individual variability and extracting features that can explain trialwise variability. The behavioral results showed that at the inter-individual level, the rare group of perceivers responded to the McGurk illusory /ta/ percept far less than the frequent group reported in our previous study (Kumar et al., 2020). This is because the frequent group of the McGurk perceivers are more likely to focus on the annotator's mouth. And, since the mouth of the annotator carries the visual speech information to induce the McGurk effect, there is a strong correlation between the perception of McGurk and mouth-looking time (Gurler D. et al., 2015; Brown V.A. et al., 2018; Figure 3a). Also, the rare group of perceivers reported far greater to the unisensory /pa/ (or auditory response) percept than the frequent group at the inter-trial level. This might be because a rare perceiver resolves the AV mismatch by focusing more on the auditory percept rather than the visually 
dominated (here $/ k a /$ ) percepts, which is usually considered an integrated percept (Morris Fernández et al., 2017; Figure 3b).

Traditionally, studies examine neural oscillations (or power) across different frequency bands defined as theta $(4-7 \mathrm{~Hz})$, alpha $(8-12 \mathrm{~Hz})$, beta $(15-30 \mathrm{~Hz})$, and gamma $(31-45 \mathrm{~Hz})$ band oscillations. However, such studies have failed to assess whether the oscillations (or rhythmic activity over a narrowband frequency range) are genuinely present or whether these power changes are just the change in arrhythmic or aperiodic components over which the oscillations manifest. In particular, there is a surprising knowledge gap in addressing this issue in the context of multisensory speech processing. Therefore, we aimed at understanding both aperiodic and periodic components of both pre-and-post stimulus oscillatory features during multisensory processing to address inter-trial and inter-individual variability of speech perception. In our study, the PSDs computed were parametrized to separate the rhythmic activity (periodic oscillations) from aperiodic $1 / f$ modulations for both the rare and frequent groups of perceivers to capture significant differences between them because a change in either or both the periodic and aperiodic component can reflect several different physiological processes (Donoghue et al., 2020). Moreover, oscillations observed in EEG/MEG signals are also a product of network interactions that result in functional connectivity dynamics. So, to capture the dynamic interactions at the sensor-level we estimated the global coherence. Our study captures the differences in the global coherence patterns between the rare and frequent group of perceivers at the prestimulus duration, indicating how the prestimulus brain state can reflect subjectwise and trial-wise variability in the perception of the McGurk illusion. We validated the functional connectivity for both the groups also at the source level and saw differences in the connectivity strength between the brain regions contributing majorly during the prestimulus brain state. The key 
findings of our study are: (1) Rare group of perceivers have a higher alpha and beta rhythmic power and a lower beta and gamma aperiodic component than the frequent group before the McGurk stimulus onset - neural signatures defining the inter-subject variability; (2) Contrastingly, however, at the inter-trial level we observed that the rare group had a lower alpha and beta periodic power but a higher alpha, beta, and gamma aperiodic component than the frequent group of perceivers; (3) Rare perceivers had a higher prestimulus theta, alpha, and beta coherence followed by a lower gamma coherence as compared to the frequent group which persisted even during the poststimulus duration, indicating different functional connectivity dynamics between the two groups that remains consistent throughout the experiment; and finally (4) The rare perceivers elicited higher cross-hemispheric connectivity (imaginary coherence) as compared to the frequent group at the source-level across different frequency bands.

\subsection{Parameterized power further explains the prestimulus brain dynamics of the two groups of perceivers.}

Periodic or rhythmic neural oscillations are considered as true oscillatory power, and in our study, they signify that both groups have a different cognitive or behavioral state. At the inter-individual level, we observed a higher prestimulus alpha and beta periodic power for the rare group suggesting a lower probability for multisensory integration resulting in illusion or non-illusion. Recent studies have reported reduced central alpha and beta band power during the perception of rubber hand illusion (Evans and Blanke 2013; Rao and Kayser 2017). Their work supports our results where the rare group had a higher beta power over the central sensors during the prestimulus duration, suggesting that a high prestimulus beta power over central sensors might dictate that a participant might be a rare perceiver of the McGurk illusion. For the poststimulus periodic power, we observed that the rare 
group had a higher poststimulus alpha and beta power followed by reduced gamma power, stating that multisensory integration resulting in illusion or non-illusion is associated with reduced perceptual processing for the rare group of perceivers (Mishra et al., 2007; Lange et al., 2011). Moreover, prestimulus alpha power fluctuations have also been linked to attention modulations, especially in the visual area (Haegens et al., 2012; Jones et al., 2010). We observed a higher alpha power over the occipito-parietal sensors for the rare group indicating that the posterior alpha rhythms might be aiding in visual attention shift (Romei V., Gross J., and Thut G. 2010).

On the contrary, at the inter-trial level, we observed a higher prestimulus beta periodic oscillatory power for the frequent group of perceivers before the participant perceived the illusory /ta/ or unisensory $/ p a /$ percept or congruent $/ p a /, / t a /$, and $/ k a /$. Our results partially coincide with the work of Keil and others (2012). They reported an increase in beta-band power in the STG, precuneus, and right frontal cortex prior to McGurk illusory percept. Here, in our study, we see that at the wholebrain sensor-scale, the periodic oscillations of the frequent group capture higher beta-band oscillations. The higher beta power sustains the illusory /ta/ and unisensory $/ \mathrm{pa} /$ response in the prestimulus duration, indicating that the beta band activity arises from brain regions specific to multisensory integration that may or may not lead to illusory percept. We backed this result with our sensor-level power distributions, where we reported that the frequent group of perceivers had maximum beta power over right central and centro-parietal sensors in the prestimulus duration before illusory $/ \mathrm{ta}$ / response. These results suggest that the parietal brain area (where precuneus lies) might contribute the most to illusory percept, especially among frequent perceivers. Whereas, before the unisensory $/ p a /$ response, the frequent group of perceivers had maximum beta power over the frontal, fronto-central, and central sensors suggesting that the frontal cortex might be contributing the most 
beta power in the prestimulus duration before unisensory / $\mathrm{pa} /$ response in anticipation of the incoming sensory stimuli. We also observed a higher alpha power for the frequent group before the McGurk illusory / $\mathrm{ta} /$ percept. Keil and others reported that only prestimulus beta power correlated with the perception of the McGurk illusion but no influence of alpha oscillations. However, we see that the frequent perceiver had significantly higher alpha oscillations before and after the illusion perception and not before or after the unisensory (or non-illusory) response to the McGurk stimulus. This might indicate that along with prestimulus beta, even alpha oscillations seem to modulate functional networks underlying illusion perception and that parametrizing the power spectrum brings out the effect of alpha activity in the perception of the McGurk illusion. Finally, the periodic component was further characterized in terms of center-frequency, broadband power (adjusted power), and bandwidth for both the groups, and it was observed that the parameters were significantly different between the two groups of perceivers (Supplementary Figure 5a). This suggests that parameters that define the periodic component contribute to the significant changes in power for both groups of perceivers.

Furthermore, the aperiodic (or arrhythmic) component of the power spectrum has been associated with modulations in cognitive states (Podvalny E. et al., 2015; He B. et al., 2010), aging (Voytek B. et al., 2015), and the excitation/inhibition balance of local neuronal populations (Manning et al., 2009; Ray \& Maunsell, 2011; Gao R. et al., 2017). Our results for the aperiodic component at the inter-individual level suggest that the rare perceivers had a lower prestimulus beta and gamma-band activity than the frequent group before the illusory and non-illusory stimulus was presented (Figure 4b, Supplementary Figure 2a). However, at the inter-trial level, we observed a higher beta and gamma-band activity for all stimulus responses and a higher alpha-band activity for McGurk illusory $/ \mathrm{ta} /$ and congruent $/ \mathrm{ta} /$ conditions for the rare perceivers (Figure 6b, Supplementary Figure 4a). 
These results suggest that the aperiodic component of prestimulus PSDs is subject-specific (interindividual variability) and remains more or less consistent across different stimulus conditions (congruent and incongruent). The aperiodic component also varies between trials, which might contribute to the inter-trial variability among McGurk perceivers. The aperiodic component was further characterized in terms of exponent (slope) and offset (of the broadband power of the power spectrum). It was observed that at the inter-individual level and inter-trial level, the prestimulus offset was significantly different between the two groups of perceivers (Figure 5, Supplementary Figure 5a), suggesting that the rare and frequent group of perceivers' offset is contributing to the significant changes in their aperiodic component. Also, the offset might reflect differences in neuronal population spiking patterns (Manning et al., 2009; Miller et al., 2012) of a rare perceiver from a frequent one which might contribute to modulations in multisensory perception.

\subsection{Global coherence as a functional connectivity marker}

We computed the global coherence to understand the functional connectivity dynamics between sensors during the prestimulus and poststimulus duration. At the inter-individual level, we observed that the rare perceivers had significantly higher prestimulus theta, alpha, and beta coherence followed by a lower gamma coherence, which was preserved even after the stimulus presentation (poststimulus) for incongruent and congruent conditions. Higher theta coherence for the rare group might reflect an enhanced cognitive control process (Cooper et al., 2015) that might prepare for upcoming stimulus. Modulations in alpha-band coherence have been associated with central executive processes of working memory (Sauseng et al., 2005). In our previous study, we reported that alpha coherence is involved in cross-modal integration and that it might be a plausible neuromarker for between-group variability. We further support these results with our prestimulus 
alpha coherence differences between the two groups indicating that the prestimulus dynamic state might define perceptual experiences. Moreover, beta coherence has been associated with top-down multisensory processing and perception (Wang, 2010; Hipp et al., 2011). Therefore, a higher prestimulus and poststimulus beta coherence for the rare perceivers might suggest a more robust hierarchical modulation of sensory and motor regions by prefrontal and frontal brain areas that might orchestrate multisensory processing. Finally, gamma coherence is associated with stimulus selection by attention (Hipp et al., 2011), which means that a heightened gamma coherence might reflect the involvement of the visual attention areas. In our study, we capture a higher gamma coherence for the frequent group of perceivers, indicating that they focused more on the visual cue rather than the auditory stimulus, making them more prone to the illusion. Overall, our study suggests that functional connectivity dynamics are pretty robust throughout multisensory processing and can act as neuronal markers to predict the McGurk illusion's perception.

\subsection{Rare perceivers elicited higher cross-hemispheric connectivity (imaginary coherence) than}

\section{the frequent group at the source across different frequency bands.}

We further isolated the sources that were governing the functional connectivity dynamics during the prestimulus and poststimulus duration. We found that the rare group had more active brain regions involved than the frequent group before and after the stimulus onset. However, brain regions majorly involved during incongruent stimuli were common between the two groups. Those regions include the superior temporal gyrus (STG; BA 22), premotor area (BA 6), precuneus (BA 7), Broca's area (speech area; BA 44, 45), and angular gyrus (language processing; BA 39) as reported previously (Jones J. \& Callan D. 2003; Keil et al., 2012). The lateral prefrontal cortex (BA 46) was also involved except for frequent perceivers during the prestimulus duration. In our study, we observed that along 
with the multisensory integration regions like STG, frontal areas like the anterior prefrontal cortex are also involved, which suggests top-down processing of the illusion. We computed the functional connectivity at different frequency bands to observe the pattern in which these regions interact between the two groups of perceivers. Overall, the rare perceivers had more cross-hemispheric interactions between the frontal regions and speech areas and the multisensory integration regions, suggesting that stronger cross-hemispheric interactions make one less prone to the illusion.

Finally, our study suggests that the neural signatures in the prestimulus duration can explain interindividual and inter-trial variability in illusory perception and that the response to an AV-stimuli can more or less be predicted. For the first time, our study has separately looked at the rhythmic oscillations, and arrhythmic components of the bandpass filtered power spectrum during a multisensory illusion paradigm. In addition to this, our study has shown differences in functional connectivity dynamics at the sensor-level between the rare and frequent group of illusory perceivers which indicates on the plausible differences in the source connectivity between the two groups of perceivers. Our source-level connectivity further supports the hypothesis. We found that rare perceivers have more cross hemispheric interaction between the multisensory integration regions like STG and prefrontal brain areas. However, the direction of the flow of information between these regions are beyond the scope of this study. To understand the information directionality, one might be to look at the phase-amplitude CFC (Cross Frequency Coupling) to understand neuronal modulations and communications (Canolty \& Knight, 2010) or perform granger causality (Dhamala et al., 2008) on the source times-series. These estimations can aid in understanding how differences in functional dynamics can aid in different perceptual experience. 


\section{Conclusion}

Overall, this study suggests that perception, in general, is a subjective experience and whether an individual will perceive a stimulus or not greatly depends on the underlying strength of the neuronal connections in the brain regions responsible for the perception. And that prestimulus oscillations might aid in predicting the response for the upcoming McGurk or any multisensory stimuli. 


\section{Acknowledgments}

This study was supported by NBRC Core funds, Ramalingaswami Fellowships (Department of Biotechnology, Government of India) to DR (BT/RLF/Re-entry/07/2014). DR was supported by SR/CSRI/21/2016 extramural grant from the Department of Science and Technology, Ministry of Science and Technology, Govt. India.

\section{Data and code availability}

The EEG data recorded and the codes used for the analysis in this study will be shared on reasonable request. All request emails are to be made to the corresponding author.

\section{Author contributions}

VS conceptualized, analyzed data, and wrote the manuscript; VK, AB, DR designed and recorded the data; $\mathrm{AB}$ reviewed the data collected, analyzed, and wrote the manuscript; and DR conceptualized, reviewed data collected, analyzed and wrote the manuscript.

\section{Conflict of interest}

All the authors declare no conflict of interest. 


\section{References}

Balazs, S., Kermanshahi, K., Binder, H., Rattay, F., \& Bodis-Wollner, I. (2016). Gamma-band modulation and coherence in the EEG by involuntary eye movements in patients in unresponsive wakefulness syndrome. Clinical EEG and neuroscience, 47(3), 196-206.

Bechtold, Bastian, 2016. Violin Plots for Matlab, Github Project https://github.com/bastibe/Violinplot-Matlab,

Benoit, M. M., Raij, T., Lin, F. H., Jääskeläinen, I. P., \& Stufflebeam, S. (2010). Primary and multisensory cortical activity is correlated with audiovisual percepts. Human brain mapping, 31(4), 526-538.

Bokil, H., Andrews, P., Kulkarni, J. E., Mehta, S., \& Mitra, P. P. (2010). Chronux: a platform for analyzing neural signals. Journal of neuroscience methods, 192(1), 146-151.

Bressler, S. L., \& Menon, V. (2010). Large-scale brain networks in cognition: emerging methods and principles. Trends in cognitive sciences, 14(6), 277-290.

Brown, V. A., Hedayati, M., Zanger, A., Mayn, S., Ray, L., Dillman-Hasso, N., \& Strand, J. F. (2018). What accounts for individual differences in susceptibility to the McGurk effect? PloS one, 13(11), e0207160.

Bullock, T. H., Mcclune, M. C. \& Enright, J. T. Are the electroencephalograms mainly rhythmic? Assessment of periodicity in wide-band time series. Neuroscience 121, 233-252 (2003).

Buzsáki, G., \& Draguhn, A. (2004). Neuronal oscillations in cortical networks. Science, 304(5679), 1926-1929.

Buzsáki, G., Anastassiou, C. A. \& Koch, C. The origin of extracellular fields and currents_EEG, ECoG, LFP and spikes. Nat. Rev. Neurosci. 13, 407-420 (2012).

Buzsáki, G., Logothetis, N., \& Singer, W. (2013). Scaling brain size, keeping timing: evolutionary preservation of brain rhythms. Neuron, 80(3), 751-764.

Canolty, R. T., \& Knight, R. T. (2010). The functional role of cross-frequency coupling. Trends in cognitive sciences, 14(11), 506-515.

Cimenser, A., Purdon, P. L., Pierce, E. T., Walsh, J. L., Salazar-Gomez, A. F., Harrell, P. G., ... \& Brown, E. N. (2011). Tracking brain states under general anesthesia by using global coherence analysis. Proceedings of the National Academy of Sciences, 108(21), 8832-8837.

Cole, S. \& Voytek, B. Cycle-by-cycle analysis of neural oscillations. J. Neurophysiol. 122, 849-861 (2019).

Cooper, P. S., Wong, A. S., Fulham, W. R., Thienel, R., Mansfield, E., Michie, P. T., \& Karayanidis, F. (2015). Theta frontoparietal connectivity associated with proactive and reactive cognitive control processes. Neuroimage, 108, 354-363. 
Delorme, A., \& Makeig, S. (2004). EEGLAB: an open source toolbox for analysis of single-trial EEG dynamics including independent component analysis. Journal of neuroscience methods, 134(1), 9-21.

Dhamala, M., Rangarajan, G., \& Ding, M. (2008). Analyzing information flow in brain networks with nonparametric Granger causality. Neuroimage, 41(2), 354-362.

DOI: $10.5281 /$ zenodo.4559847

Donoghue, T., Haller, M., Peterson, E. J., Varma, P., Sebastian, P., Gao, R., ... \& Voytek, B. (2020). Parameterizing neural power spectra into periodic and aperiodic components. Nature neuroscience, 23(12), 1655-1665.

Engel, A. K., Fries, P., \& Singer, W. (2001). Dynamic predictions: oscillations and synchrony in top-down processing. Nature Reviews Neuroscience, 2(10), 704-716.

Evans, N., \& Blanke, O. (2013). Shared electrophysiology mechanisms of body ownership and motor imagery. Neuroimage, 64, 216-228.

Fonseca, L. C., Tedrus, G. M., Rezende, A. L. R., \& Giordano, H. F. (2015). Coherence of brain electrical activity: a quality of life indicator in Alzheimer's disease? Coerência da atividade elétrica cerebral: indicador da qualidade de vida na doença de Alzheimer?. Arquivos de Neuro-psiquiatria, 73, 396-401.

Freeman, W. J., \& Zhai, J. (2009). Simulated power spectral density (PSD) of background electrocorticogram (ECoG). Cognitive neurodynamics, 3(1), 97-103.

Gao, R., Peterson, E. J., \& Voytek, B. (2017). Inferring synaptic excitation/inhibition balance from field potentials. Neuroimage, $158,70-78$.

Giuseppe Cardillo (2021). mwwtest (https://github.com/dnafinder/mwwtest), GitHub. Retrieved June 12, 2021.

Gross, J., Kujala, J., Hämäläinen, M., Timmermann, L., Schnitzler, A., \& Salmelin, R. (2001). Dynamic imaging of coherent sources: studying neural interactions in the human brain. Proceedings of the National Academy of Sciences, 98(2), 694-699. Journal of neuroscience methods, 163(1), 161-175.

Gurler, D., Doyle, N., Walker, E., Magnotti, J., \& Beauchamp, M. (2015). A link between individual differences in multisensory speech perception and eye movements. Attention, Perception, \& Psychophysics, 77(4), 13331341. https://doi.org/10.3758/s13414-014-0821-1

Haegens, S., Luther, L., \& Jensen, O. (2012). Somatosensory anticipatory alpha activity increases to suppress distracting input. Journal of cognitive neuroscience, 24(3), 677-685.

He, B. J. (2014). Scale-free brain activity: past, present, and future. Trends in cognitive sciences, 18(9), 480-487. 
He, B. J., Zempel, J. M., Snyder, A. Z. \& Raichle, M. E. The temporal structures and functional significance of scale-free brain activity. Neuron 66, 353-369 (2010).

Hipp, J. F., Engel, A. K., \& Siegel, M. (2011). Oscillatory synchronization in large-scale cortical networks predicts perception. Neuron, 69(2), 387-396.

Jensen, O., Bonnefond, M., \& VanRullen, R. (2012). An oscillatory mechanism for prioritizing salient unattended stimuli. Trends in cognitive sciences, 16(4), 200-206.

Jones, J. A., \& Callan, D. E. (2003). Brain activity during audiovisual speech perception: an fMRI study of the McGurk effect. Neuroreport, 14(8), 1129-1133.

Jones, S. R., Kerr, C. E., Wan, Q., Pritchett, D. L., Hämäläinen, M., \& Moore, C. I. (2010). Cued spatial attention drives functionally relevant modulation of the mu rhythm in primary somatosensory cortex. Journal of Neuroscience, 30(41), $13760-13765$.

Kayser, S. J., McNair, S. W., \& Kayser, C. (2016). Prestimulus influences on auditory perception from sensory representations and decision processes. Proceedings of the National Academy of Sciences, 113(17), 4842-4847.

Keil, J., Müller, N., Ihssen, N., \& Weisz, N. (2012). On the variability of the McGurk effect: audiovisual integration depends on prestimulus brain states. Cerebral Cortex, 22(1), 221-231.

Kumar, G. V., Halder, T., Jaiswal, A. K., Mukherjee, A., Roy, D., \& Banerjee, A. (2016). Large scale functional brain networks underlying temporal integration of audiovisual speech perception: An EEG study. Frontiers in psychology, $7,1558$.

Kumar, V. G., Dutta, S., Talwar, S., Roy, D., \& Banerjee, A. (2020). Biophysical mechanisms governing large-scale brain network dynamics underlying individual-specific variability of perception. European Journal of Neuroscience, 52(7), 3746-3762.

Lange, J., Oostenveld, R., \& Fries, P. (2011). Perception of the touch-induced visual double-flash illusion correlates with changes of rhythmic neuronal activity in human visual and somatosensory areas. Neuroimage, 54(2), 1395-1405.

Lansbergen, M. M., Arns, M., van Dongen-Boomsma, M., Spronk, D. \& Buitelaar, J. K. The increase in theta/beta ratio on resting-state EEG in boys with attention-deficit/hyperactivity disorder is mediated by slow alpha peak frequency. Prog. Neuropsychopharmacol. Biol. Psychiatry 35, 47-52 (2011).

Mallick, D. B., Magnotti, J. F., \& Beauchamp, M. S. (2015). Variability and stability in the McGurk effect: contributions of participants, stimuli, time, and response type. Psychonomic bulletin \& review, 22(5), 1299-1307.

Manning, J. R., Jacobs, J., Fried, I. \& Kahana, M. J. Broadband shifts in local field potential power spectra are correlated with single-neuron spiking in humans. J. Neurosci. 29, 13613-13620 (2009). 
Maris, E., Schoffelen, J. M., \& Fries, P. (2007). Nonparametric statistical testing of coherence differences. Journal of neuroscience methods, 163(1), 161-175.

McGurk, H., \& MacDonald, J. (1976). Hearing lips and seeing voices. Nature, 264(5588), 746-748.

Miller, K. J. et al. Human motor cortical activity is selectively phase-entrained on underlying rhythms. PLoS Comput. Biol. 8, e1002655 (2012)

Minor, M. W. (1970). Experimenter-expectancy effect as a function of evaluation apprehension. Journal of Personality and Social Psychology, 15(4), 326.

Mishra, J., Martinez, A., Sejnowski, T. J., \& Hillyard, S. A. (2007). Early cross-modal interactions in auditory and visual cortex underlie a sound-induced visual illusion. Journal of Neuroscience, 27(15), 4120-4131.

Mitra, P. (2007). Observed brain dynamics. Oxford University Press.

Moris Fernandez, L., Macaluso, E., \& Soto-Faraco, S. (2017). Audiovisual integration as conflict resolution: The conflict of the McGurk illusion. Human brain mapping, 38(11), 5691-5705.

Nath, A. R., \& Beauchamp, M. S. (2012). A neural basis for interindividual differences in the McGurk effect, a multisensory speech illusion. Neuroimage, 59(1), 781-787.

Nath, A. R., Fava, E. E., \& Beauchamp, M. S. (2011). Neural correlates of interindividual differences in children's audiovisual speech perception. Journal of Neuroscience, 31(39), 13963-13971.

Nolte, G., Bai, O., Wheaton, L., Mari, Z., Vorbach, S., \& Hallett, M. (2004). Identifying true brain interaction from EEG data using the imaginary part of coherency. Clinical neurophysiology, 115(10), 2292-2307.

Oostenveld, R., Fries, P., Maris, E., \& Schoffelen, J. M. (2011). FieldTrip: open source software for advanced analysis of MEG, EEG, and invasive electrophysiological data. Computational intelligence and neuroscience, 2011.

Podvalny, E., Noy, N., Harel, M., Bickel, S., Chechik, G., Schroeder, C. E., ... \& Malach, R. (2015). A unifying principle underlying the extracellular field potential spectral responses in the human cortex. Journal of neurophysiology, 114(1), $505-519$.

Railo, H., Koivisto, M., \& Revonsuo, A. (2011). Tracking the processes behind conscious perception: a review of event-related potential correlates of visual consciousness. Consciousness and cognition, 20(3), 972-983.

Rao, I. S., \& Kayser, C. (2017). Neurophysiological correlates of the rubber hand illusion in late evoked and alpha/beta band activity. Frontiers in human neuroscience, 11, 377.

Ray, S., \& Maunsell, J. H. (2011). Different origins of gamma rhythm and high-gamma activity in macaque visual cortex. PLoS biology, 9(4), e1000610. 
Romei, V., Gross, J., \& Thut, G. (2010). On the role of prestimulus alpha rhythms over occipito-parietal areas in visual input regulation: correlation or causation?. Journal of Neuroscience, 30(25), 8692-8697.

Sauseng, P., Klimesch, W., Schabus, M., \& Doppelmayr, M. (2005). Fronto-parietal EEG coherence in theta and upper alpha reflect central executive functions of working memory. International journal of Psychophysiology, 57(2), 97-103.

Spadone, S., Croce, P., Zappasodi, F., \& Capotosto, P. (2020). Prestimulus EEG microstates correlate with anticipatory alpha desynchronization. Frontiers in Human Neuroscience, 14.

Strand, J., Cooperman, A., Rowe, J., \& Simenstad, A. (2014). Individual differences in susceptibility to the McGurk effect: links with lipreading and detecting audiovisual incongruity. Journal of Speech, Language, and Hearing Research, 57(6), 2322-2331.

Van Dijk, H., Schoffelen, J. M., Oostenveld, R., \& Jensen, O. (2008). Prestimulus oscillatory activity in the alpha band predicts visual discrimination ability. Journal of Neuroscience, 28(8), 1816-1823.

Van Dijk, H., Schoffelen, J. M., Oostenveld, R., \& Jensen, O. (2008). Prestimulus oscillatory activity in the alpha band predicts visual discrimination ability. Journal of Neuroscience, 28(8), 1816-1823.

Varela, F., Lachaux, J. P., Rodriguez, E., \& Martinerie, J. (2001). The brainweb: phase synchronization and large-scale integration. Nature reviews neuroscience, 2(4), 229-239.

Voytek, B., Kramer, M. A., Case, J., Lepage, K. Q., Tempesta, Z. R., Knight, R. T., \& Gazzaley, A. (2015). Age-related changes in 1/f neural electrophysiological noise. Journal of Neuroscience, 35(38), 13257-13265.

Wang, X. J. (2010). Neurophysiological and computational principles of cortical rhythms in cognition. Physiological reviews, 90(3), 1195-1268.

Waskom, M. L. (2021). Seaborn: statistical data visualization. Journal of Open Source Software, 6(60), 3021.

Wen, H., \& Liu, Z. (2016). Separating fractal and oscillatory components in the power spectrum of neurophysiological signal. Brain topography, 29(1), 13-26.

Winawer, J. et al. Asynchronous broadband signals are the principal source of the BOLD response in human visual cortex. Curr. Biol. 23, 1145-1153 (2013).

Xia, M., Wang, J., \& He, Y. (2013). BrainNet Viewer: a network visualization tool for human brain connectomics. PloS one, 8(7), e68910. 


\section{Figure Captions}

Figure 1: Schematic of the overall study. We aimed at understanding the oscillatory dynamics in the prestimulus durations between the two groups of the McGurk perceivers, rare and frequent. The participants were categorized based on their overall behavioral responses. Participants with $<50 \%$ behavioral response to the McGurk perception were considered as rare perceivers and $>50 \%$ as frequent perceivers. At the sensor level, we first computed the parameterized power to capture the oscillatory differences between the two groups at the inter-individual and inter-trial levels of variability. We further analyzed the global coherence dynamics to identify the neural signatures of the variability between the rare and frequent group of perceivers in the prestimulus domain. Finally, we identified the sources and their coherence strength (connectivity) to capture the differences in the neuronal dynamics of the two groups of perceivers that determine the individual's response to the upcoming McGurk stimulus.

Figure 2: Prestimulus time window. Example trial with a fixation cross pseudo-randomly varied between 1200 to $2800 \mathrm{~ms}$ before the onset of the audiovisual stimuli. The red box indicates the time window chosen for prestimulus epoch data of $800 \mathrm{~ms}$ duration.

Figure 3: Behavior. (a) Inter-individual variability - Propensity of McGurk effect for all the 18 participants expressed as the percentage of / ta/ percept during the presentation of the McGurk illusion. The participants were categorized into two groups: rare perceivers (red dots, $<50 \%$ ) and frequent perceivers (blue dots, $>50 \%$ ). (b) Violin plots showing inter-trial variability - Percentage of /ta/ (illusory) and /pa/ (unisensory) percept during the presentation of McGurk stimulus and the congruent 
AV stimuli (/pa/, /ta/, and / ka/) averaged over participants in the rare group of perceivers, and frequent group of perceivers. The white dot in each plot represents the median.

Figure 4: Parametrized power indicating inter-individual variability. (a) Periodic oscillations and (b) Aperiodic distributions of the rare (red line) and frequent (blue line) group of McGurk perceivers before (prestimulus, on the left) and after (poststimulus, on the right) the stimulus onset. The highlighted frequency bands specify regions where the difference between the two groups was significant. The corresponding difference in periodic or aperiodic component power is shown for the whole-brain at the sensor level using topoplots.

Figure 5: Aperiodic parameter - Offset. At the inter-individual level, only the aperiodic parameter offset showed a significant $(p=0.041)$ difference between the two groups of perceivers (violin plot, on the left). On the right, the sensor-level distribution of the offset parameter for the rare and frequent group. The difference between the offset was maximum at the $\mathrm{C} 3$ sensor.

Figure 6: Parametrized power indicating inter-trial variability. (a) Periodic oscillations and (b) Aperiodic distributions of the rare (red line) and frequent (blue line) group of McGurk/ta/ (illusory) perceivers before (prestimulus, on the left) and after (poststimulus, on the right) the stimulus onset. The highlighted frequency bands specify regions where the difference between the two groups was significant.

Figure 7: Large-scale functional connectivity. Time-averaged global coherence before (prestimulus, on the left) and after (poststimulus, on the right) McGurk stimulus onset at (a) inter-individual 
variability and (b) inter-trial variability. For inter-individual variability plots, the black dashed box indicates global coherence significantly higher for the rare group, and the black-dotted box indicates significantly lower coherence for the rare group as compared to the frequent group.

Table 1: Sources involved during the prestimulus duration. Coordinates of centroids of the reconstructed sources (clustered through k-means) involved during prestimulus duration along with their corresponding Brodmann areas for (a) rare group and (b) frequent group of perceivers.

Figure 8: Coherence strength between sources during the prestimulus duration. We computed the coherence strength of those corresponding frequency bands, which showed a significant difference in total coherence before the McGurk stimulus onset (or prestimulus) at the sensor level. The frequency bands are theta coherence for (a) rare group and (b) frequent group; alpha coherence for (c) rare and (d) frequent group; beta coherence for (e) rare and (f) frequent group; and gamma coherence for $(\mathrm{g})$ rare and $(\mathrm{h})$ frequent group of perceivers. 


\section{Supplemental Figure Captions}

Supplementary Figure 1: Periodic distributions indicating inter-individual variability. Periodic oscillations of the power spectrum for the rare group (red line) and frequent group (blue line) for congruent $/ \mathrm{pa} / \mathrm{/ta} /$, and $/ \mathrm{ka} /$ stimulus conditions during (a) prestimulus and (b) poststimulus durations with topoplots, indicating periodic differences between the two groups at frequency bands where the difference was significant between the rarely and frequently McGurk perceiving groups.

\section{Supplementary Figure 2: Aperiodic distributions indicating inter-individual variability. Aperi-}

odic oscillations of the power spectrum for the rare group (red line(s)) and frequent group (blue line(s)) for congruent $/ \mathrm{pa} / \mathrm{t} / \mathrm{ta}$, and $/ \mathrm{ka} / \mathrm{stimulus} \mathrm{conditions} \mathrm{during} \mathrm{(a)} \mathrm{prestimulus} \mathrm{and} \mathrm{(b)} \mathrm{poststim-}$ ulus durations with topoplots, indicating aperiodic differences between the two groups at frequency bands where the difference was significant between the rarely and frequently McGurk perceiving groups.

Supplementary Figure 3: Periodic distributions indicating inter-trial variability. Periodic oscillations of the power spectrum for the rare group (red line) and frequent group (blue line) for unisensory McGurk /pa/, and congruent / $\mathrm{pa} / \mathrm{/ta} /$, and $/ \mathrm{ka} /$ stimulus conditions during (a) prestimulus, and (b) poststimulus durations with shaded regions that indicate frequency bands where the difference in power was significant between the two groups of perceivers.

Supplementary Figure 4: Aperiodic distributions indicating inter-trial variability. Aperiodic oscillations of the power spectrum for the rare group (red line(s)) and frequent group (blue line(s)) for unisensory McGurk/pa/, and congruent / $\mathrm{pa} / \mathrm{t} / \mathrm{ta} /$, and $/ \mathrm{ka} /$ stimulus conditions during (a) prestimulus, 
and (b) poststimulus durations with shaded regions that indicate frequency bands where the difference in power was significant between the two groups of perceivers.

\section{Supplementary Figure 5: Parameters computed for parametrizing power using FOOOF for the}

two groups of perceivers at the inter-trial level. Violin plots showcasing whether the difference in aperiodic parameters (exponent and offset) and periodic parameters (center-frequency, adjusted power, and bandwidth) between the rare (blue violin plot) and frequent (orange violin plot) for all the stimulus conditions during (a) prestimulus and (b) poststimulus duration is significant or not.

\section{Supplementary Figure 6: Large-scale functional connectivity indicating inter-individual varia-} bility. Time-averaged global coherence for the rare group (red line) and frequent group (blue line) for congruent $/ \mathrm{pa} /, \mathrm{ta} /$, and $/ \mathrm{ka} /$ stimulus conditions during (a) pre-stimulus, and (b) post-stimulus durations. The black dashed box indicates global coherence significantly higher for the rare group, and the black-dotted box indicates significantly lower coherence for the rare group as compared to the frequent group.

Supplementary Table 1: Table summarizing the significant difference at inter-individual level, in total coherence (whether higher or lower) for the rare group as compared to the frequent group of perceivers during (a) prestimulus and (b) poststimulus for incongruent and congruent stimulus conditions.

\section{Supplementary Figure 7: Large-scale functional connectivity indicating inter-trial variability.}

Time-averaged global coherence for the rare (dotted lines) and frequent (solid lines) for congruent 
$/ p a /, / t a /$, and $/ \mathrm{ka} /$ stimulus conditions during (a) pre-stimulus, and (b) post-stimulus durations. The tables below each plot summarize the significant difference at the inter-trial level in total coherence (whether higher or lower) for the rare group as compared to the frequent group of perceivers for incongruent and congruent stimulus conditions.

Supplementary Table 2: Sources involved during the poststimulus duration. Coordinates of centroids of the reconstructed sources (clustered through k-means) involved during poststimulus duration along with their corresponding Brodmann areas for (a) rare group and (b) frequent group of perceivers.

\section{Supplementary Figure 8: Coherence strength between sources during the poststimulus duration.}

We computed the coherence strength of those corresponding frequency bands, which showed a significant difference in total coherence after the McGurk stimulus onset (or poststimulus) at the sensor level. The frequency bands are theta coherence for (a) rare group and (b) frequent group; alpha coherence for (c) rare and (d) frequent group; beta coherence for (e) rare and (f) frequent group; and gamma coherence for $(\mathrm{g})$ rare and $(\mathrm{h})$ frequent group of perceivers. 
Figures:

bioRxiv preprint doi: https://doi.org/10.1101/2022.01.20.477172; this version posted January 22, 2022. The Aperiodic parameters (which was not certified by peer review) is the author/funder, who hasogranted bioRxiv a license to display theipreprint in perpetuity. It is made available under aCC-BY 4.0 International license.
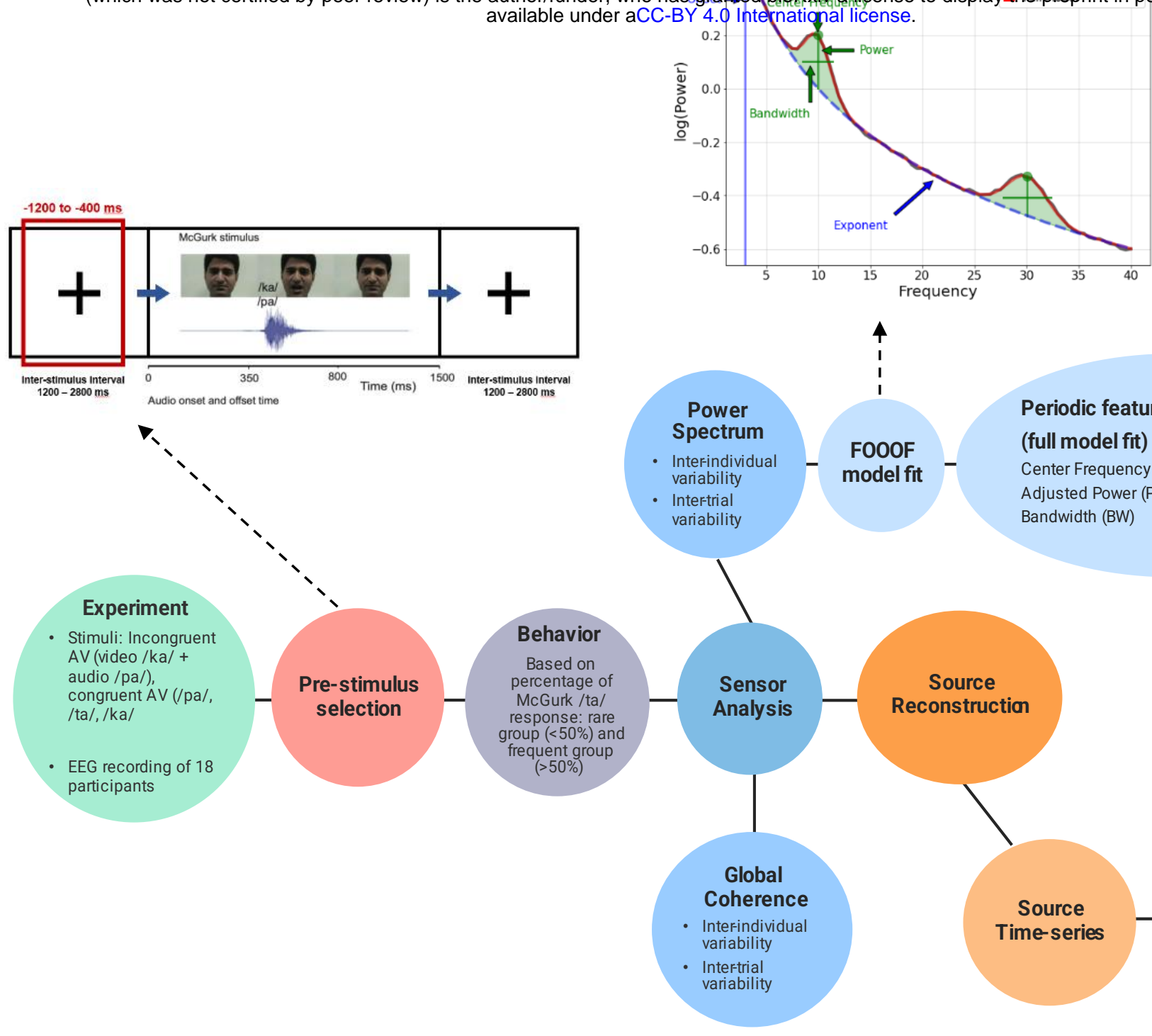

Periodic features Aperiodic fit (full model fit)

Center Frequency (CF) $\quad 1 / f$ Offset Adjusted Power (PW) $1 /$ Exponent Bandwidth (BW) (slope)

\section{Figure 1}


bioRxiv preprint doi: https://doi.org/10.1101/2022.01.20.477172; this version posted January 22, 2022. The copyright holder for this preprint (which was not certified by peer review) is the author/funder, who has granted bioRxiv a license to display the preprint in perpetuity. It is made available under aCC-BY 4.0 International license.

\section{Pre-stimulus time window}

-1200 to $-400 \mathrm{~ms}$

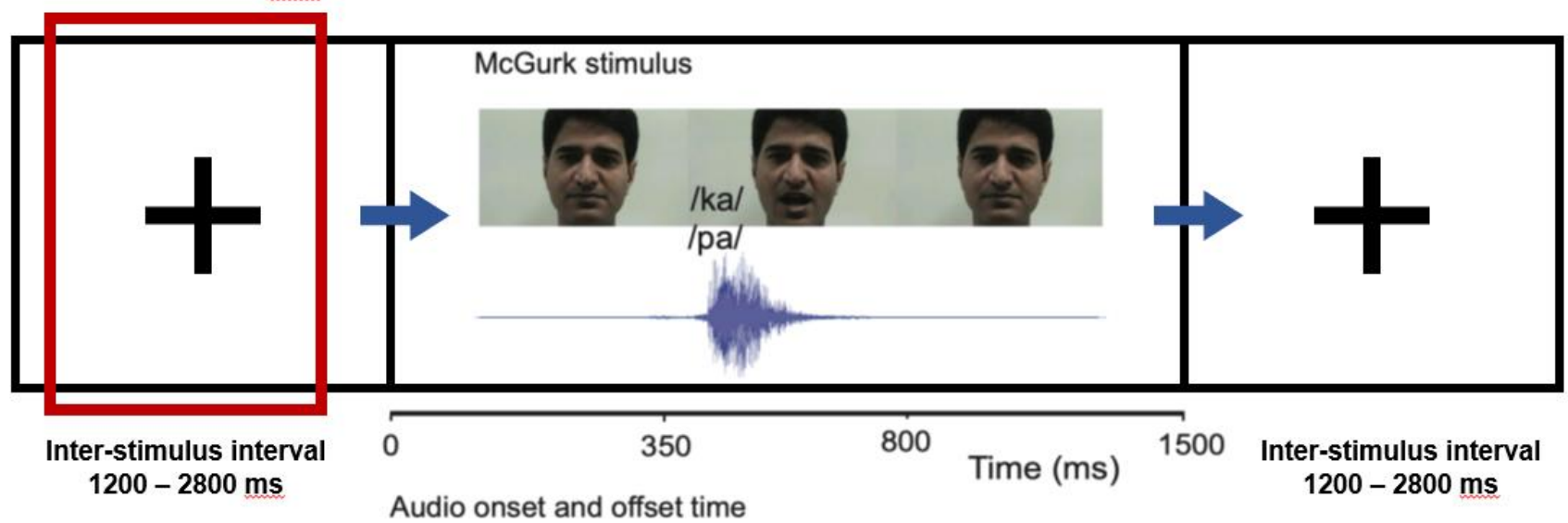

Figure 2 
bioRxiv preprint doi: https://doi.org/10.1101/2022.01.20.477172; this version posted January 22, 2022. The copyright holder for this preprint (which was not certified by peer review) is the author/funder, who has granted bioRxiv a license to display the preprint in perpetuity. It is made available under aCC-BY 4.0 International license.

\section{Behavior}

\section{(b) Inter-Trial Variability}

(a) Inter-Individual Variability

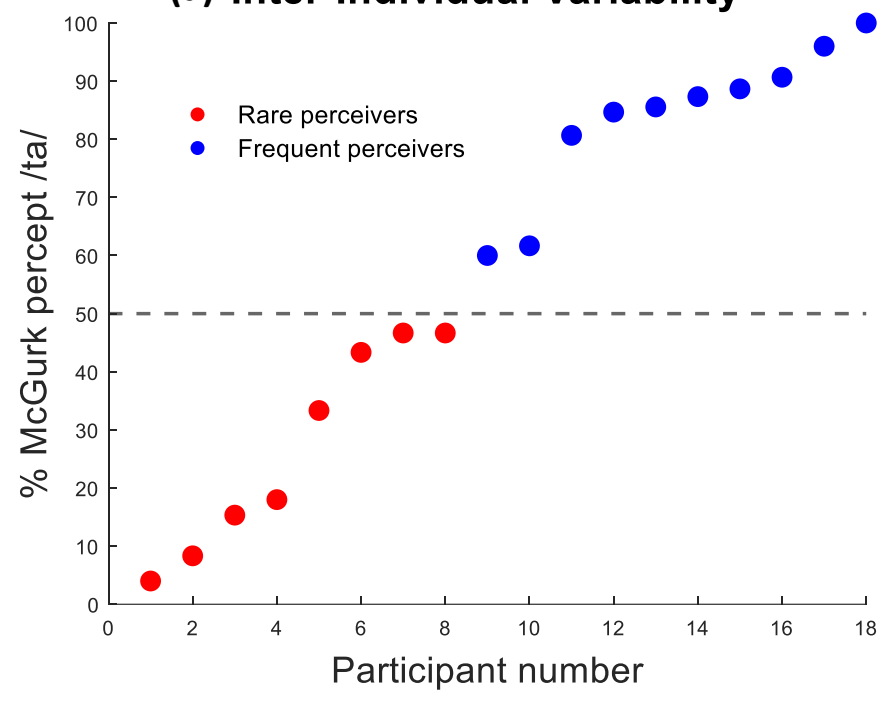

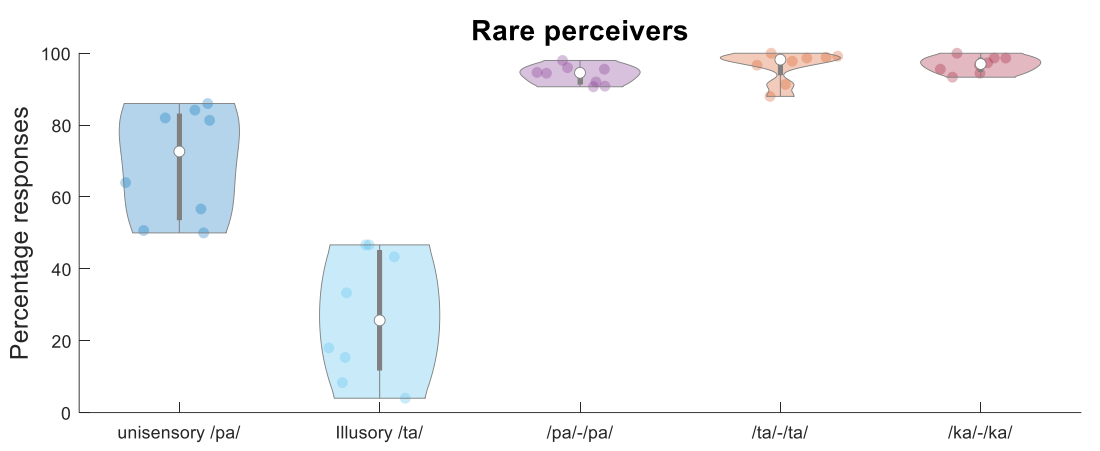

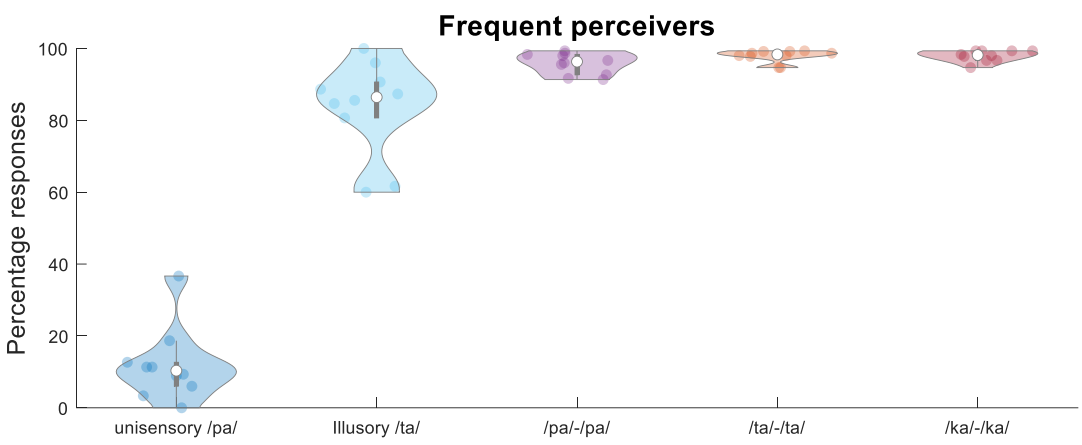

\section{Figure 3}


Inter-individual Variability

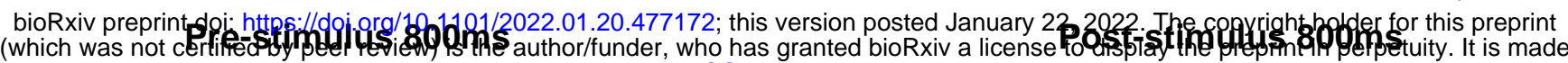
available under aCC-BY 4.0 International license.

\section{(a) Periodic Distributions}
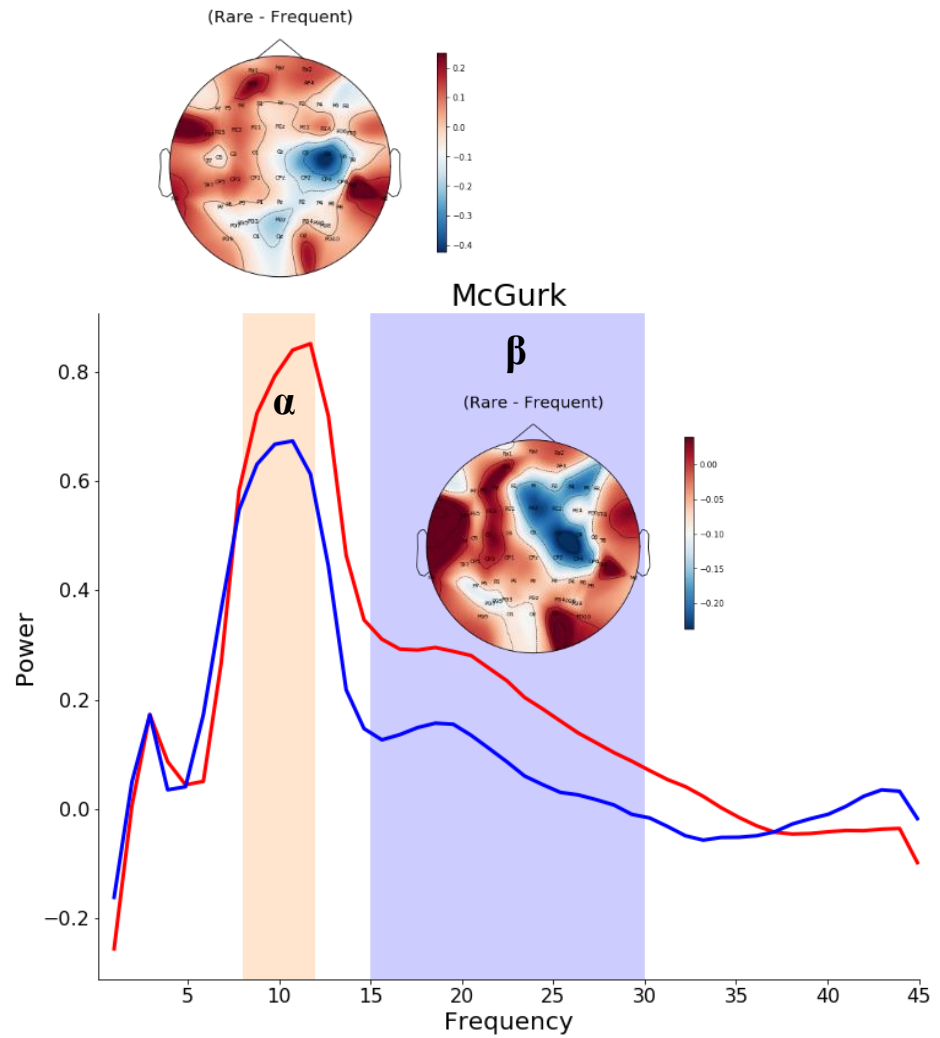
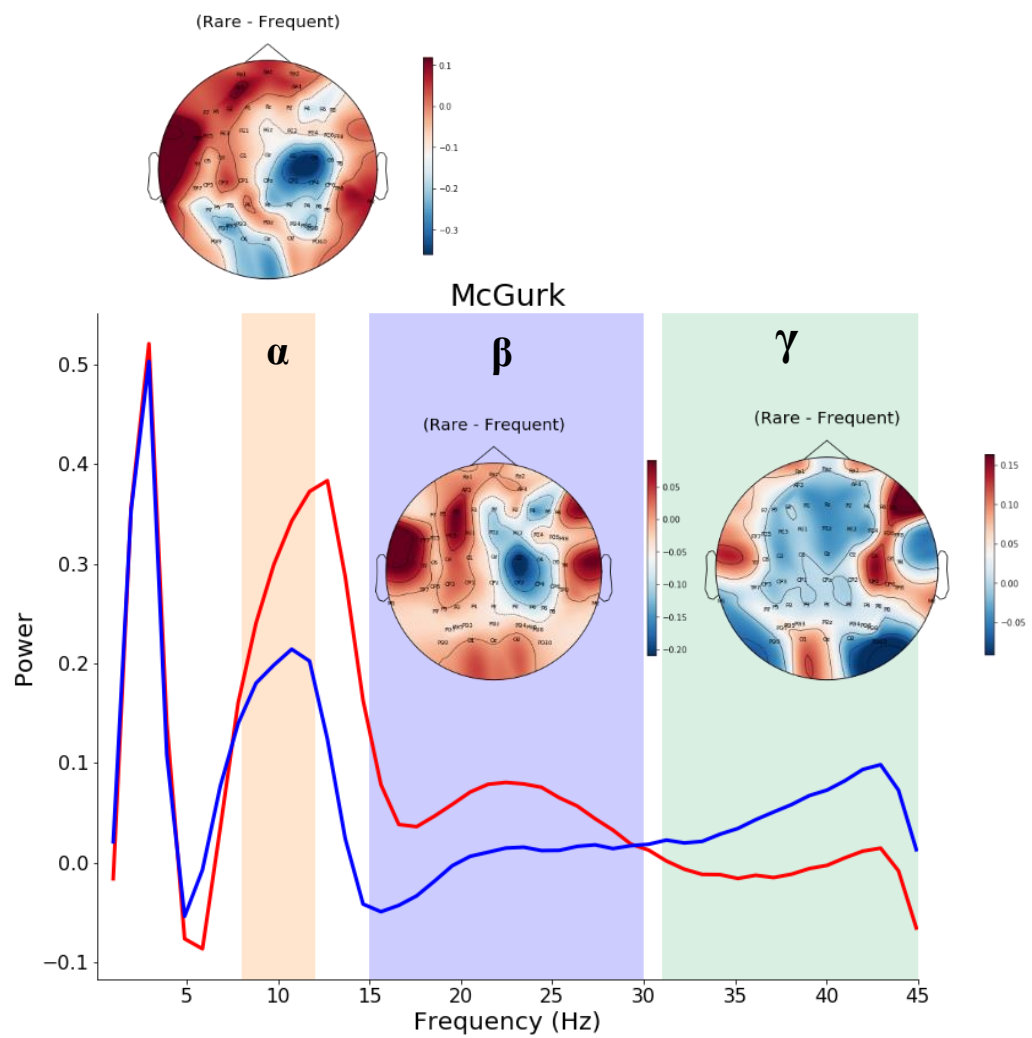

(b) Aperiodic Distributions
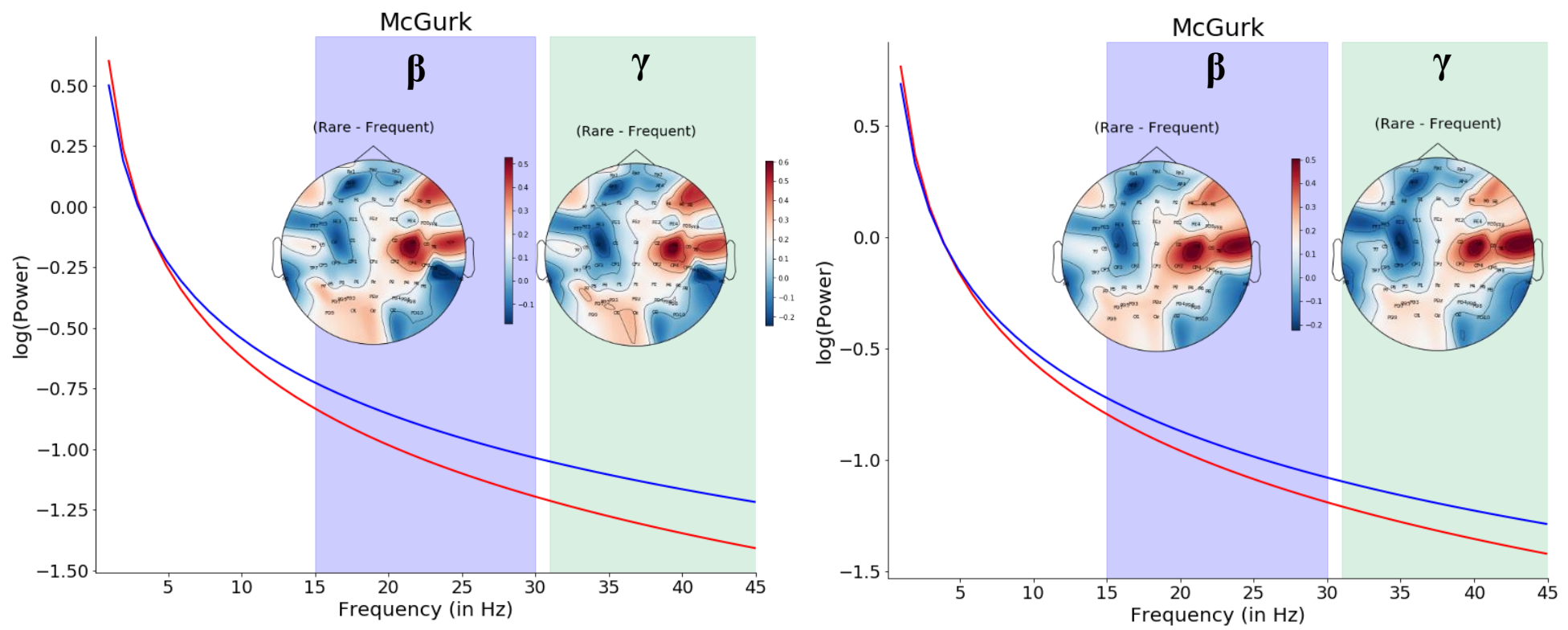

Figure 4 


\section{Inter-individual Variability}

bioRxiv preprint doi: https://doi.org/10.1101/2022.01.20.477172; this version posted January 22, 2022. The copyright holder for this preprint (which was not certified by peer review) is the author/funder, who has granted bioRxiv a license to display the preprint in perpetuity. It is made available under aCC-BY 4.0 International license.

\section{Pre-stimulus $800 \mathrm{~ms}$}
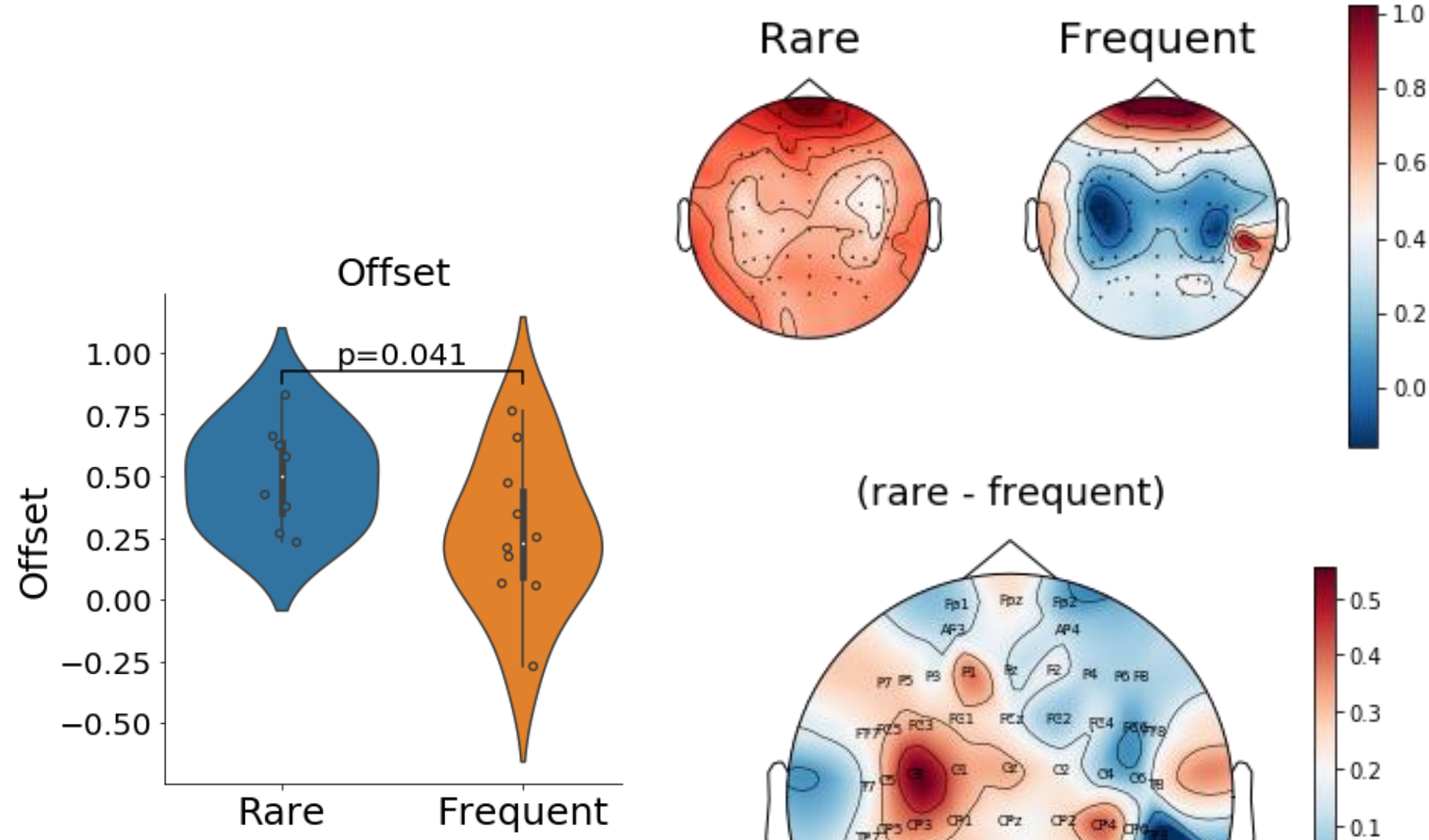

\section{(rare - frequent)}

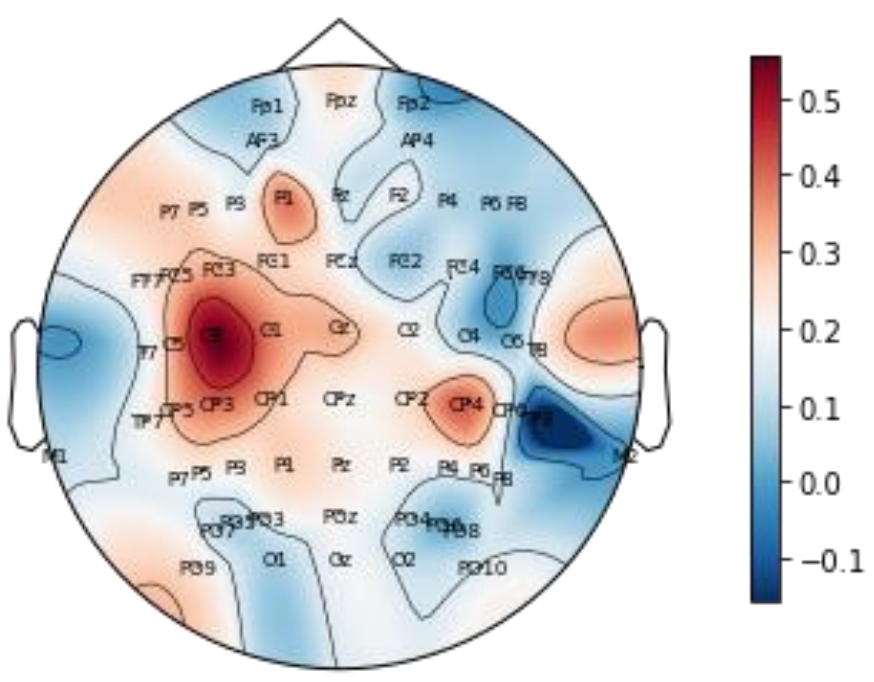

Figure 5 


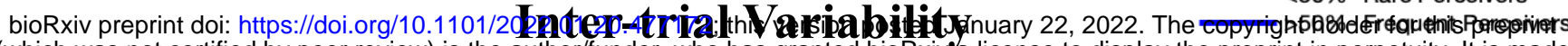
(which was not certified by peer review) is the author/funder, who has granted bioRxiva license to display the preprint in perpetuity. It is made

\section{Pre-stimulus $800 \mathrm{~ms}$} available under aCC-BY 4.0 International license.

Post-stimulus $800 \mathrm{~ms}$

(a) Periodic Distributions
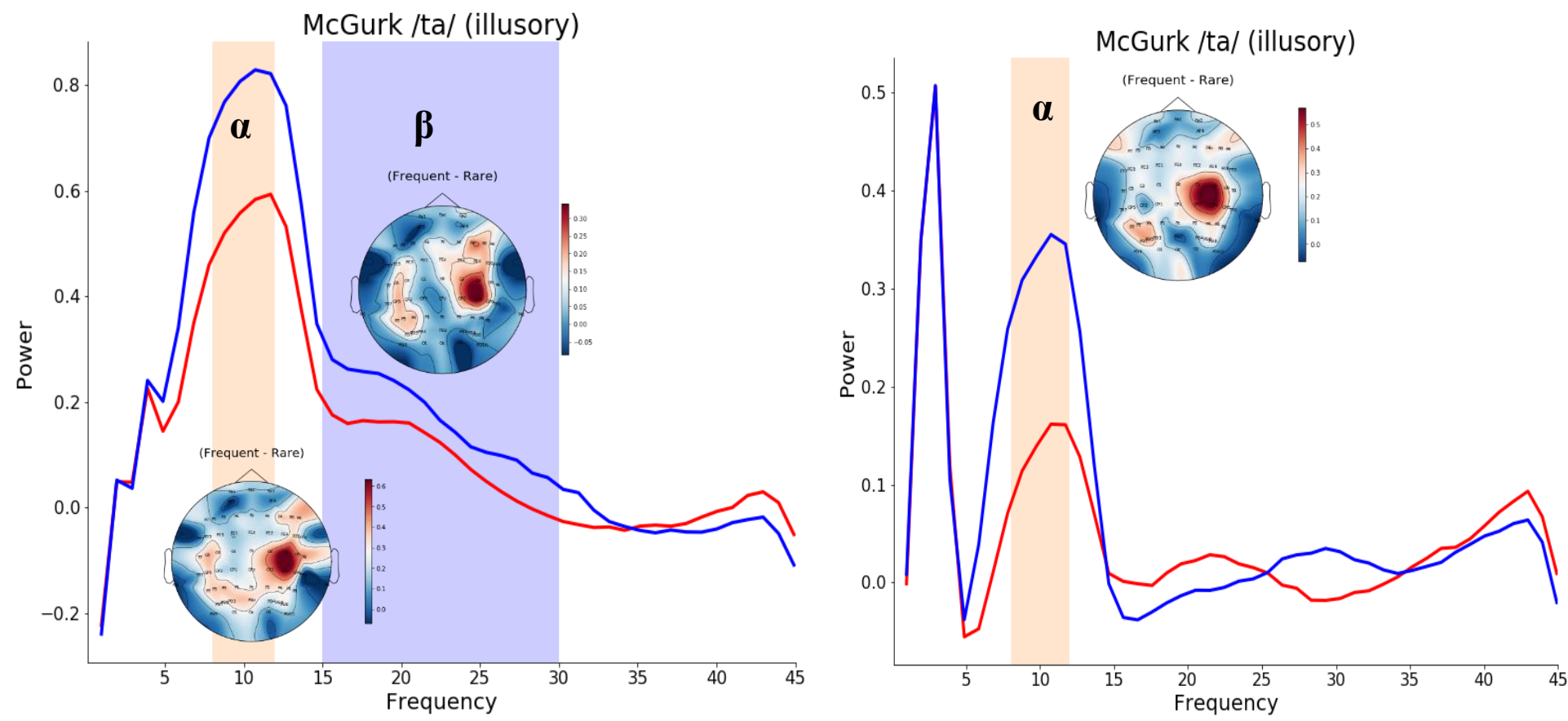

(b) Aperiodic Distributions
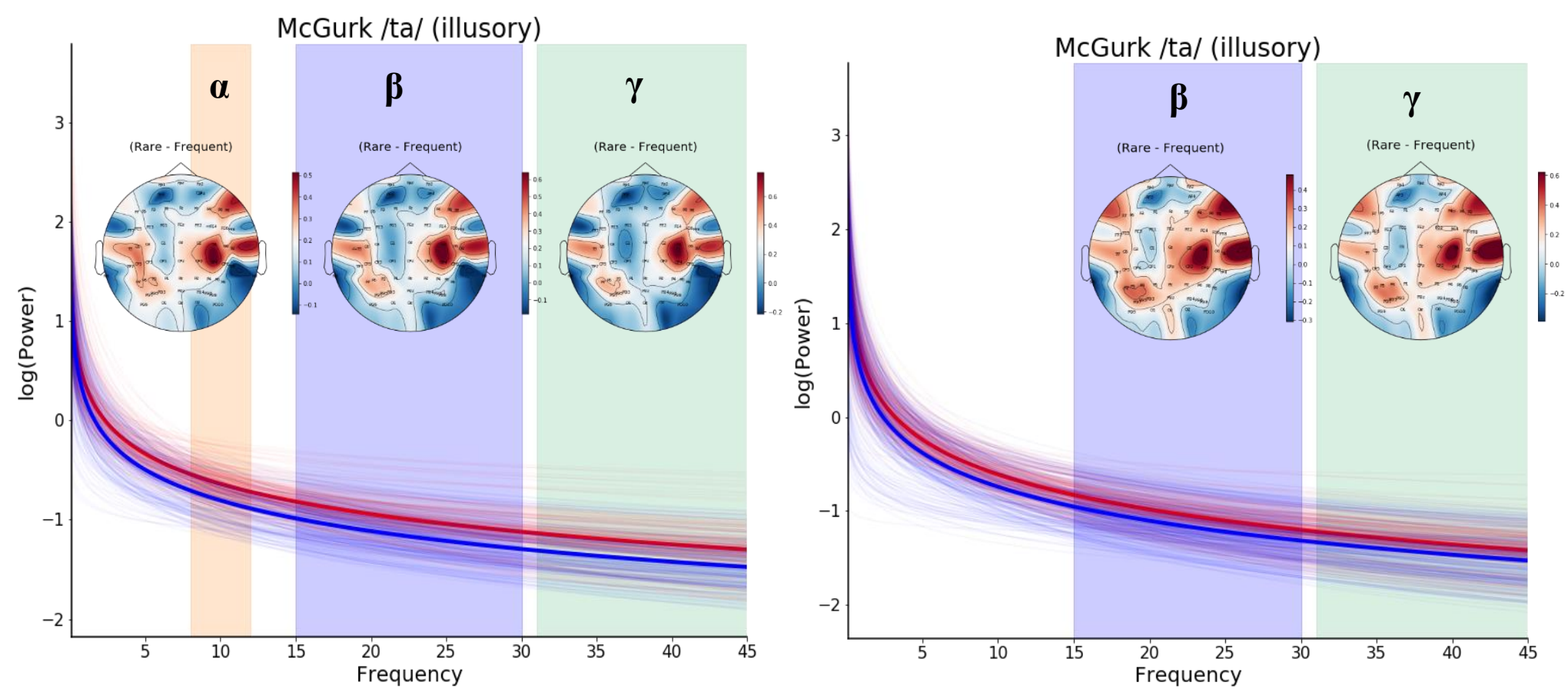

Figure 6 


\section{Global Coherence}

bioRxiv preprint doi: https://doi.org/10.1101/2022.01.20.477172; this version posted January 22, 2022. The copyright holder for this preprint

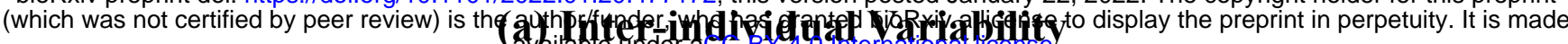

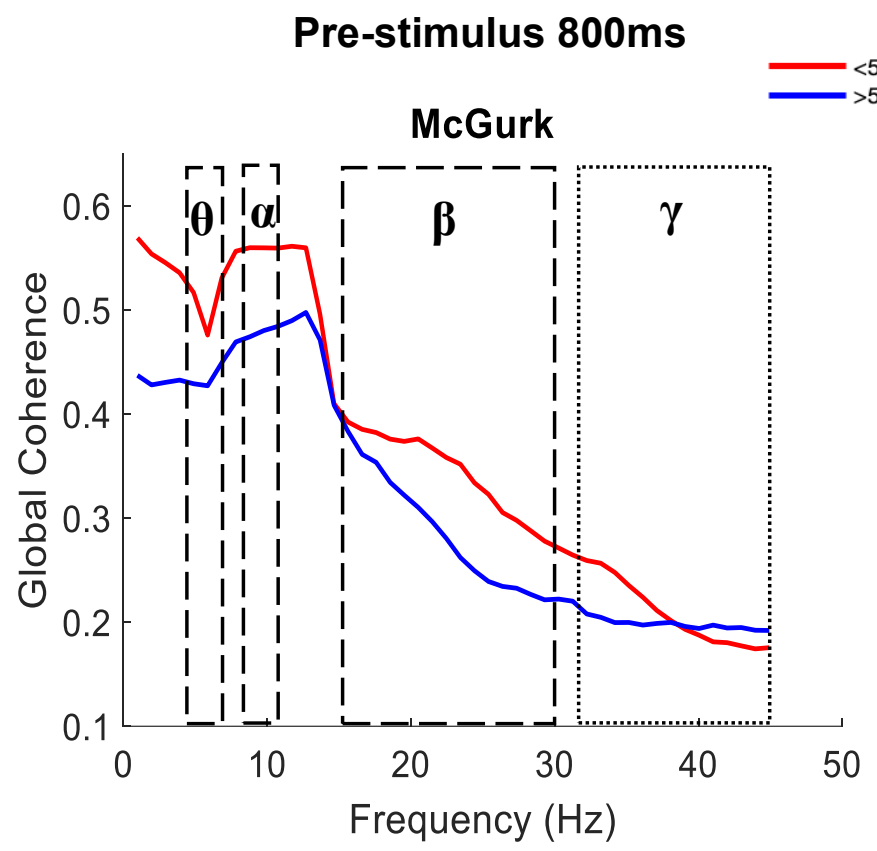

\section{Post-stimulus $800 \mathrm{~ms}$} McGurk

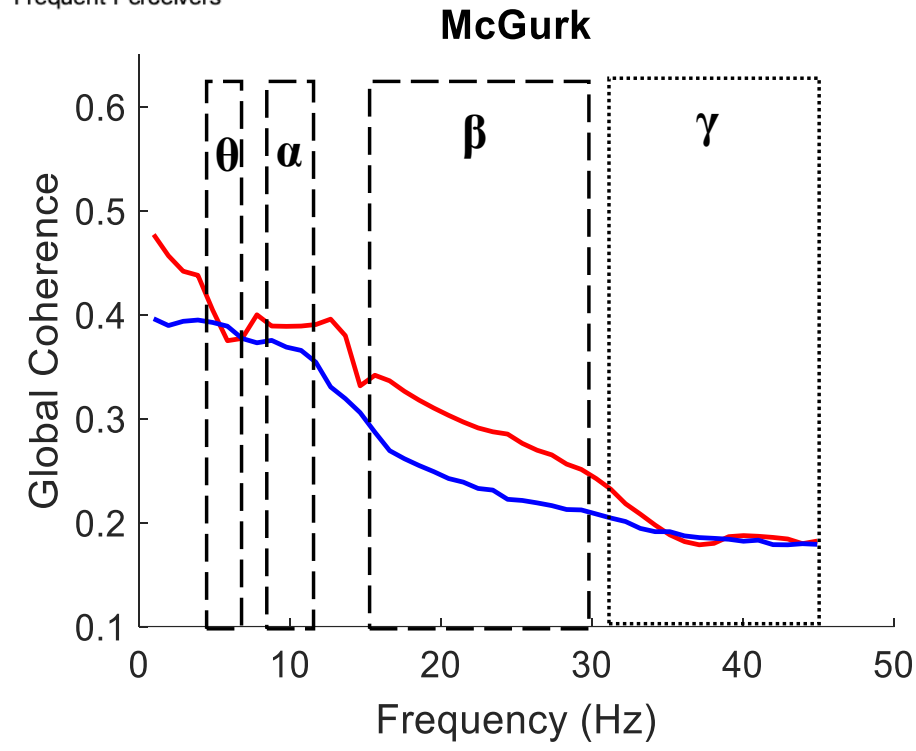

(b) Inter-trial Variability

Pre-stimulus $800 \mathrm{~ms}$
Post-stimulus $800 \mathrm{~ms}$

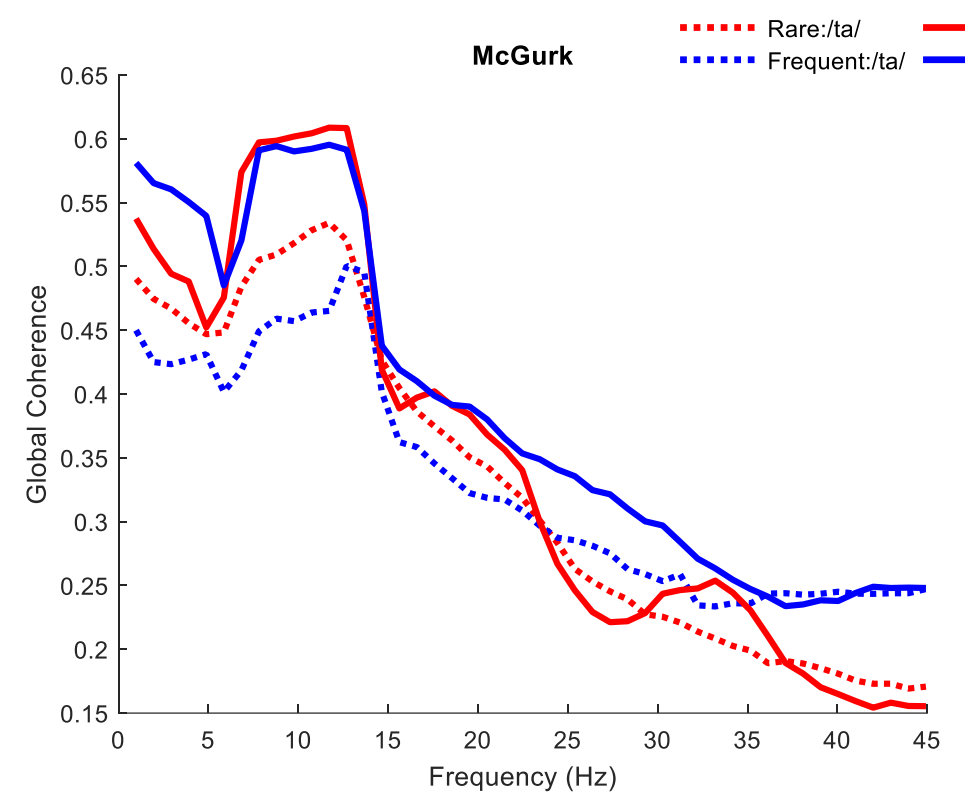

Rare:/pa/
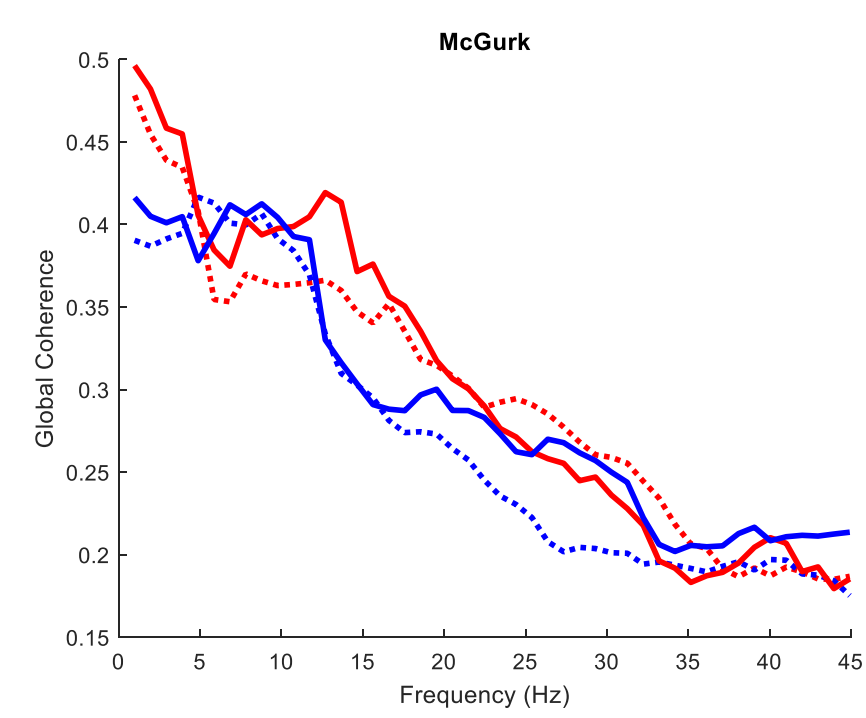

Figure 7 


\section{Sources involved in the pre-stimulus duration}

bioRxiv preprint doi: https://doi.org/10.1101/2022.01.20.477172; this version posted January 22, 2022. The copyright holder for this preprint (which was not certified by peer review) is the author/funder, who has granted bioRxiv a license to display the preprint in perpetuity. It is made (a) For the rare group of percểile

\begin{tabular}{ccccc}
\hline $\mathbf{X}$ & $\mathbf{Y}$ & $\mathbf{Z}$ & Brodmann area & Region \\
\hline 68.000 & -8.902 & 3.193 & Right-BA-22 & Right Superior Temporal Gyrus \\
-32.971 & 58.477 & 6.871 & Left-BA-10 & Left Anterior Pre-Frontal Cortex \\
32.746 & 62.178 & 10.755 & Right-BA-10 & $\begin{array}{c}\text { Right Anterior Pre-Frontal Cortex } \\
-33.095\end{array}$ \\
-3.929 & 72.254 & Left-BA-6 & Left Pre-Motor + Supplementary Motor Area \\
45.265 & 52.938 & 4.209 & Right-BA-46 & Right Dorso-lateral Pre-Frontal Cortex (lateral) \\
32.912 & -78.553 & 61.539 & Right-BA-7 & Right Visual Motor Area \\
63.694 & -54.237 & 39.584 & Right-BA-39 & Right Angular Gyrus \\
60.889 & 24.076 & 24.234 & Right-BA-9 & Right Dorso-lateral Pre-Frontal Cortex (dorsal) \\
-58.163 & 15.009 & 26.837 & Left-BA-44 & Left Broca's Opercularis (inferior frontal gyrus) \\
64.573 & 10.305 & 4.962 & Right-BA-44 & Right Broca's Opercularis (inferior frontal gyrus) \\
\hline
\end{tabular}

(b) For the frequent group of perceivers:

\begin{tabular}{ccccc}
\hline $\mathbf{X}$ & $\mathbf{Y}$ & $\mathbf{Z}$ & Brodmann area & Region \\
\hline 39.243 & 57.418 & 6.835 & Right-BA-10 & Right Anterior Pre-Frontal Cortex \\
63.435 & -54.599 & 39.618 & Right-BA-39 & Right Angular Gyrus \\
-58.202 & 14.978 & 28.360 & Left-BA-44 & Left Broca's Opercularis (inferior frontal cortex) \\
-32.559 & -4.324 & 72.984 & Left-BA-6 & Left Pre-Motor + Supplementary Motor Area \\
60.763 & 24.526 & 23.928 & Right-BA-44 & Right Broca's Opercularis (inferior frontal cortex) \\
32.458 & -78.981 & 61.285 & Right-BA-7 & Right Visual Motor Area \\
-33.409 & 58.501 & 7.337 & Left-BA-10 & Left Anterior Pre-Frontal Cortex \\
66.265 & 1.372 & 3.982 & Right-BA-22 & Right Superior Temporal Gyrus \\
\hline
\end{tabular}




\section{Pre-stimulus}

(a) Rare Group

(b) Frequent Group

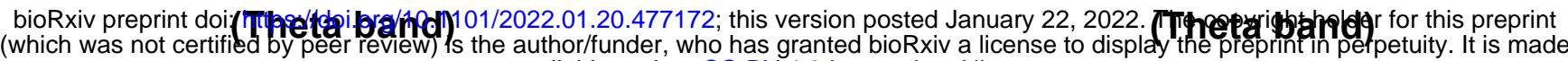
available under aCC-BY 4.0 International license.

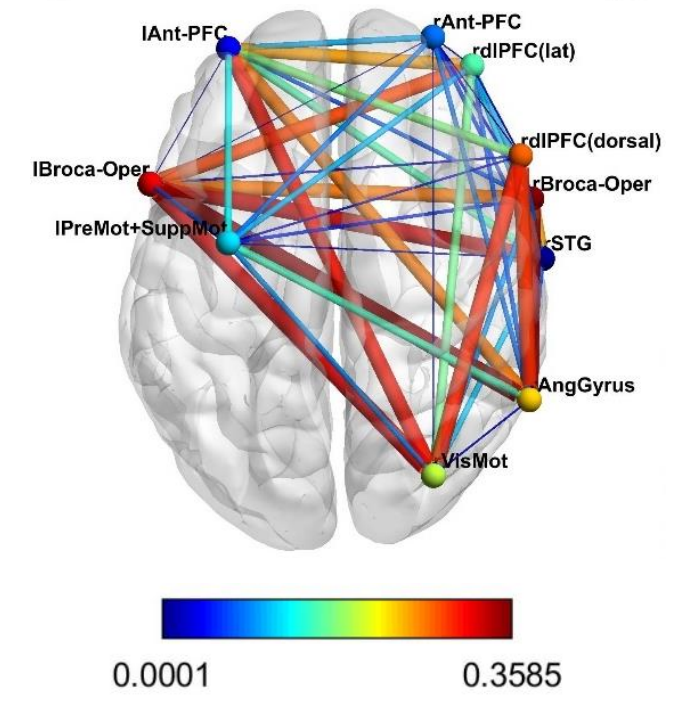

0.0001
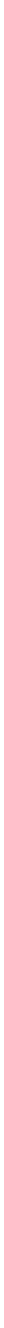

(d) Frequent Group (Alpha band)

$\mathbf{R}$

\section{(c) Rare Group
(Alpha band) \\ (c) Rare Group
(Alpha band)}

(a)
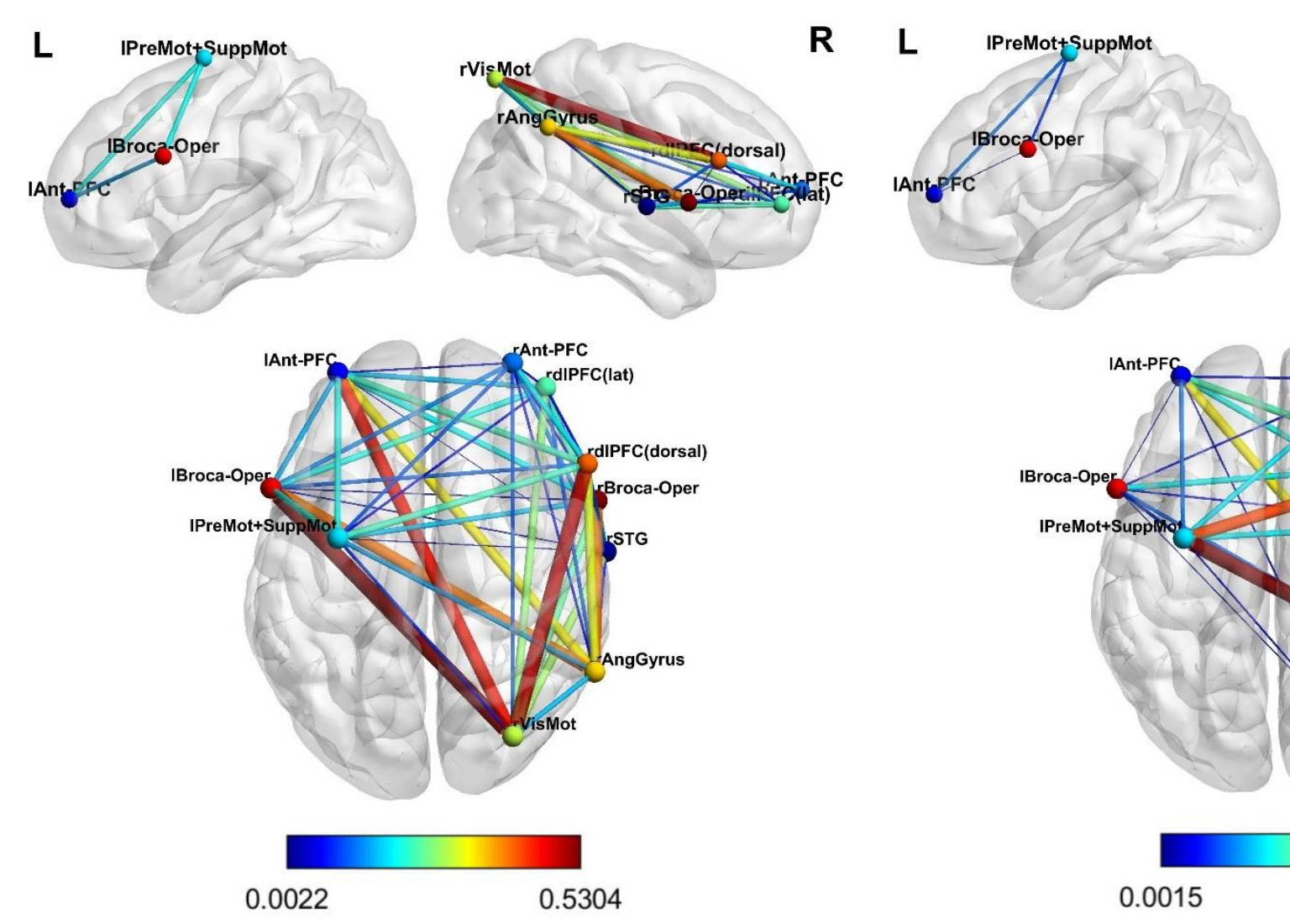

$\mathbf{R}$

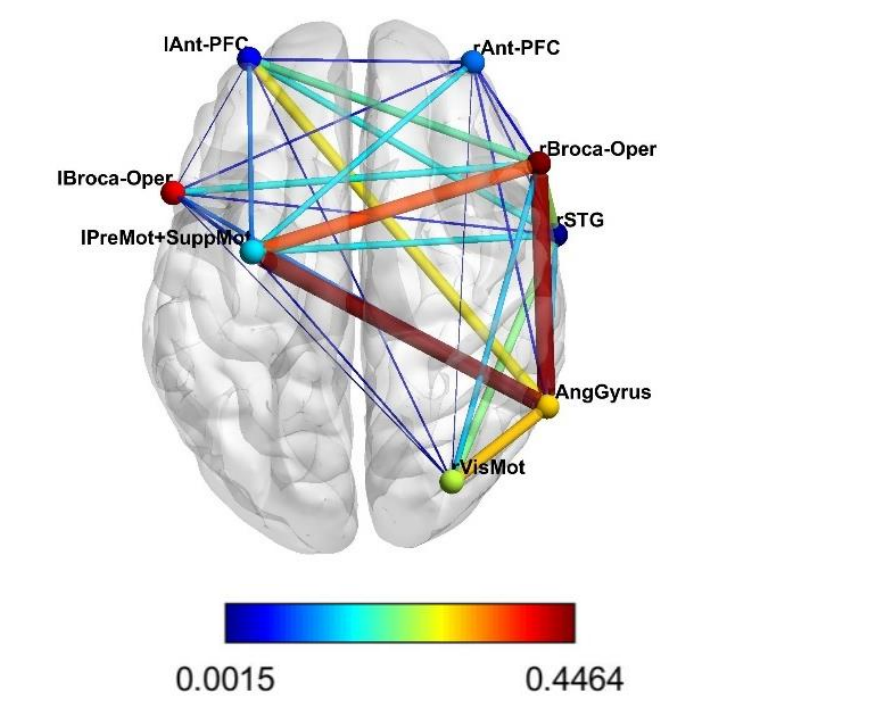

Figure 8

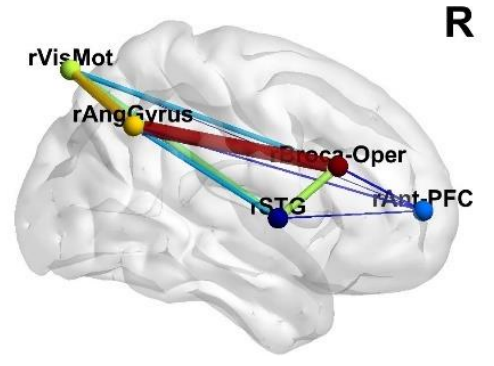

$$
\text { R }
$$

\section{.}




\section{Pre-stimulus}

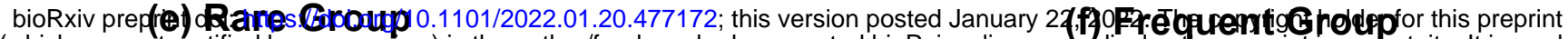
(which was not certified by peer review) is the author/funder, who has granted bioRxiv a license to display the preprint in perpetuity. It is made (Beta band) available under aCC-BY 4.0 International license.

(Beta band)
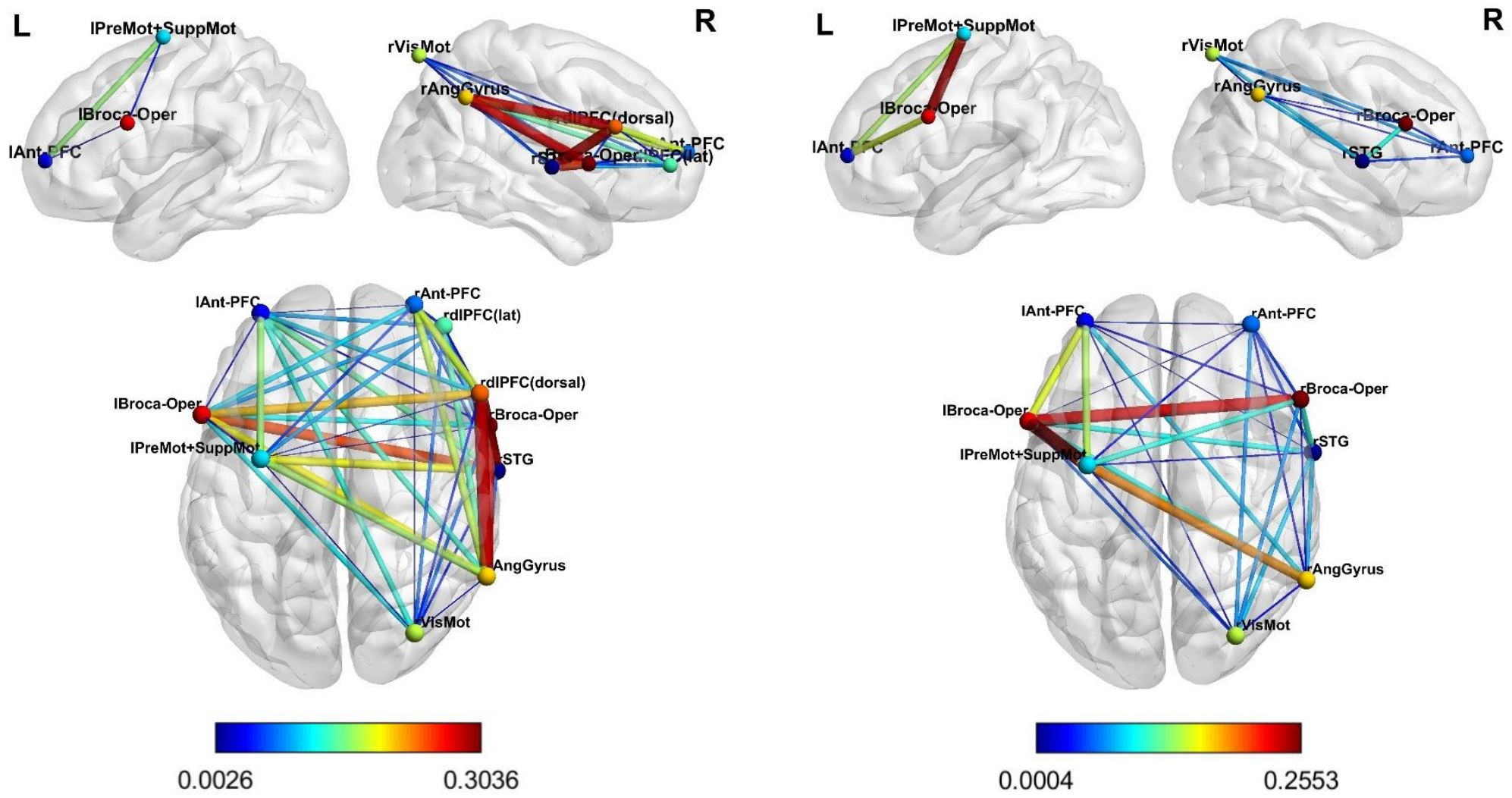

\section{(g) Rare Group (Gamma band)}

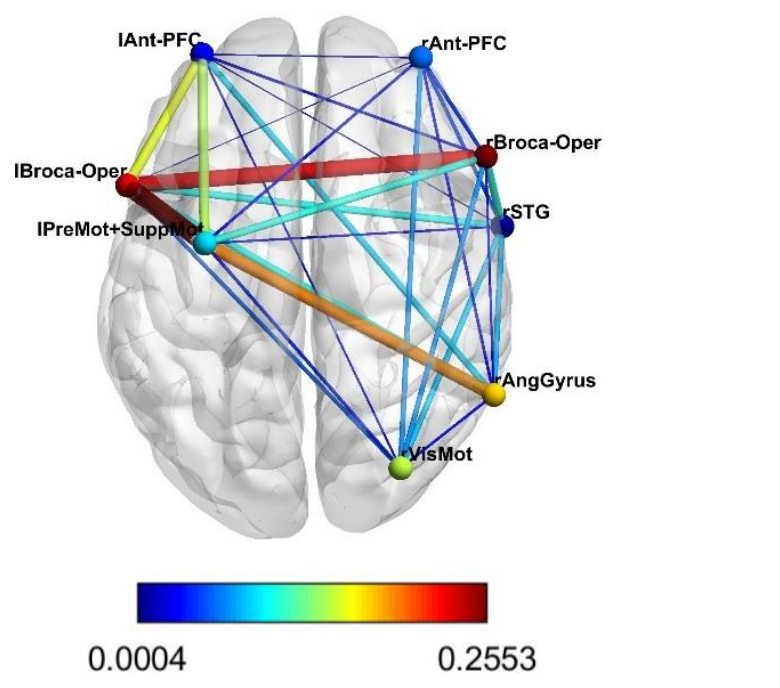

\section{(h) Frequent Group} (Gamma band)
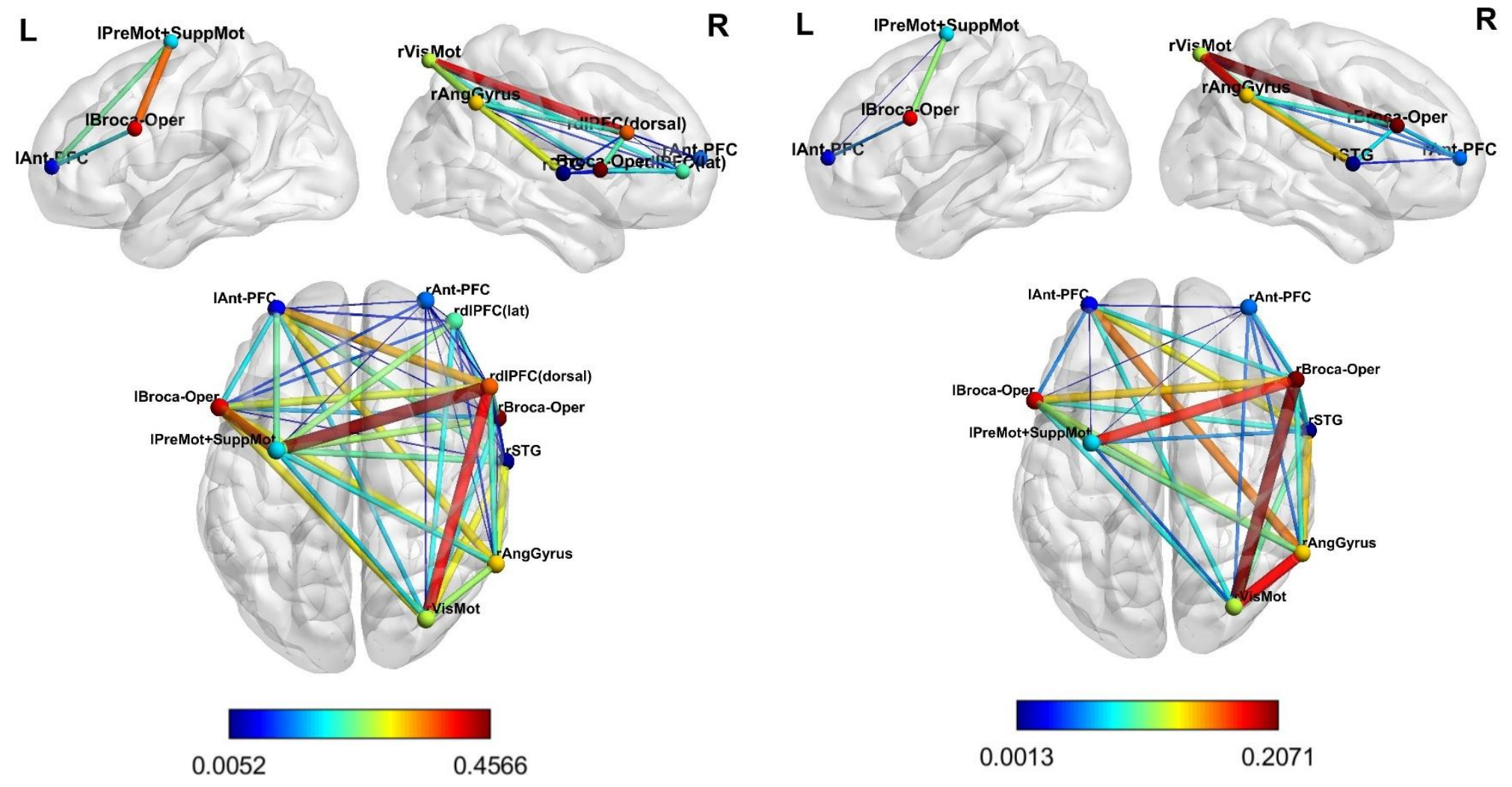

0.0013

0.2071

Figure 8 
bioRxiv preprint doi: https://doi.org/10.1101/2022.01.20.477172; this version posted January 22, 2022. The copyright holder for this preprint (which was not certified by peer review) is the author/funder, who has granted bioRxiv a license to display the preprint in perpetuity. It is made Supplementary Figures: available under aCC-BY 4.0 International license.

\section{Periodic Distributions \\ Inter-individual Variability}

$<50 \%$ - Rare Perceivers

$>50 \%$ - Frequent Perceivers (a) Pre-stimulus $800 \mathrm{~ms}$
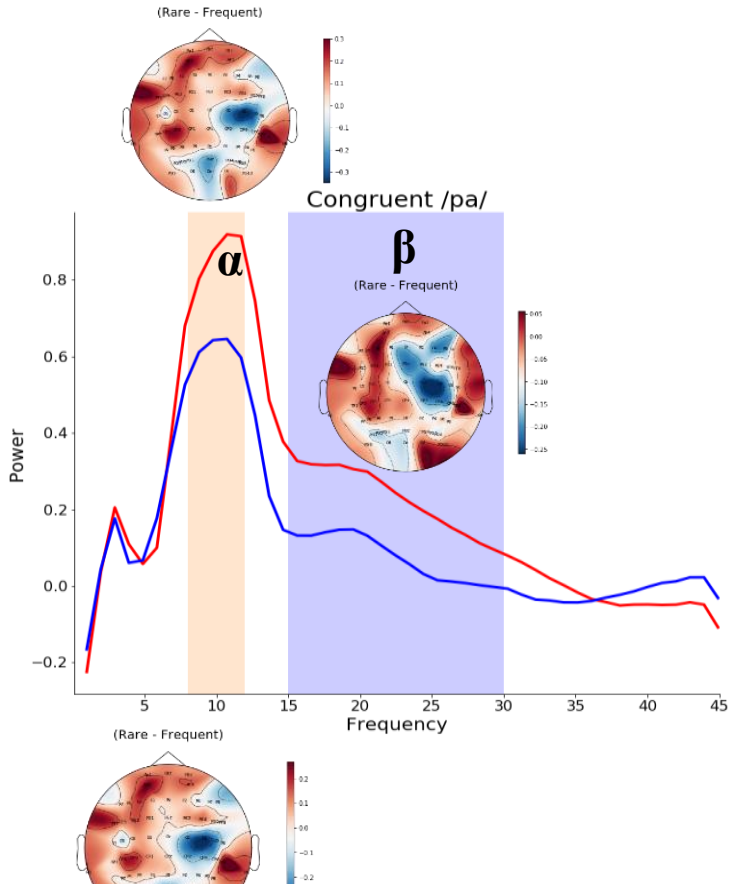

Congruent $/ \mathrm{ta} /$
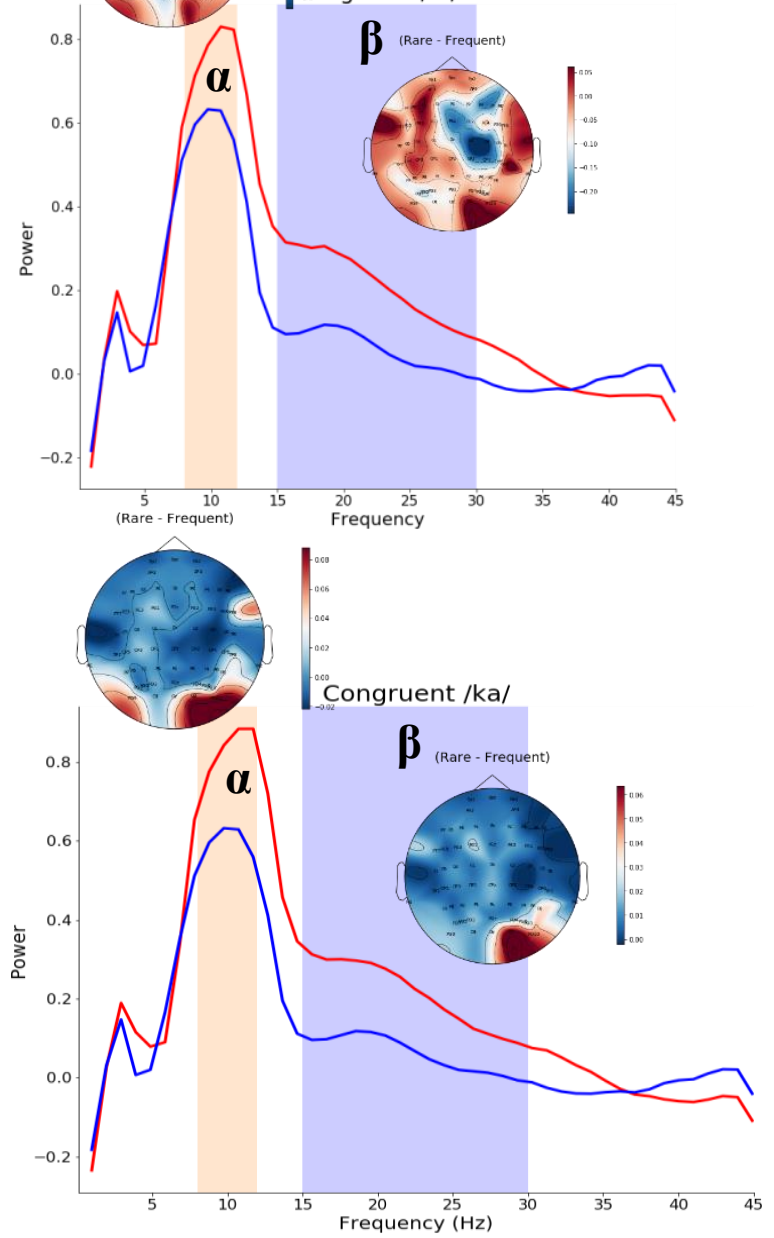

(b) Post-stimulus $800 \mathrm{~ms}$
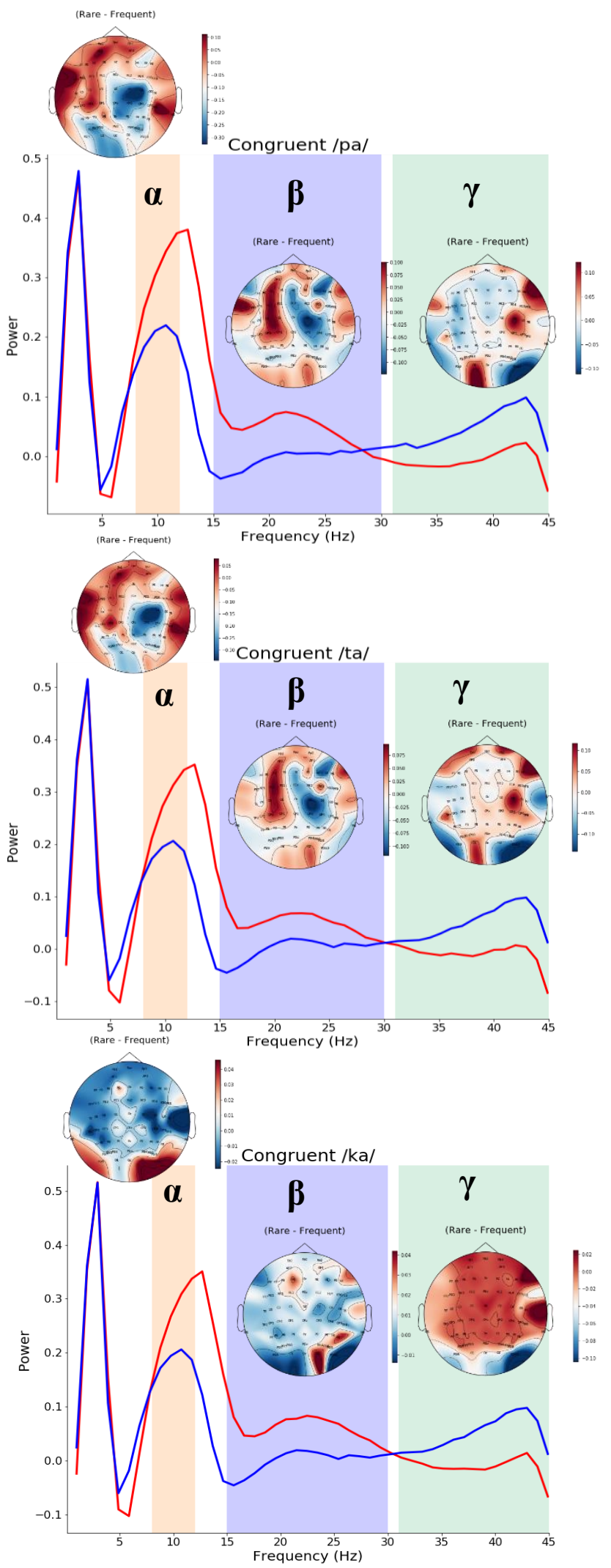
bioRxiv preprint doi: https://doi.org/10.1101/2022.dP.20.49/2; Distribution putions 22, 2022. The copyright holder for this preprint

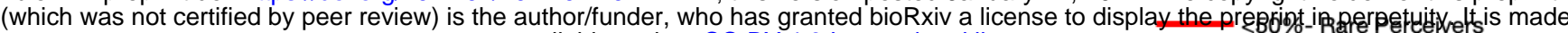

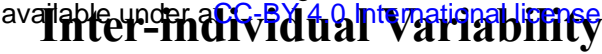
$>50 \%$ - Frequent Perceivers

(a) Pre-stimulus $800 \mathrm{~ms}$
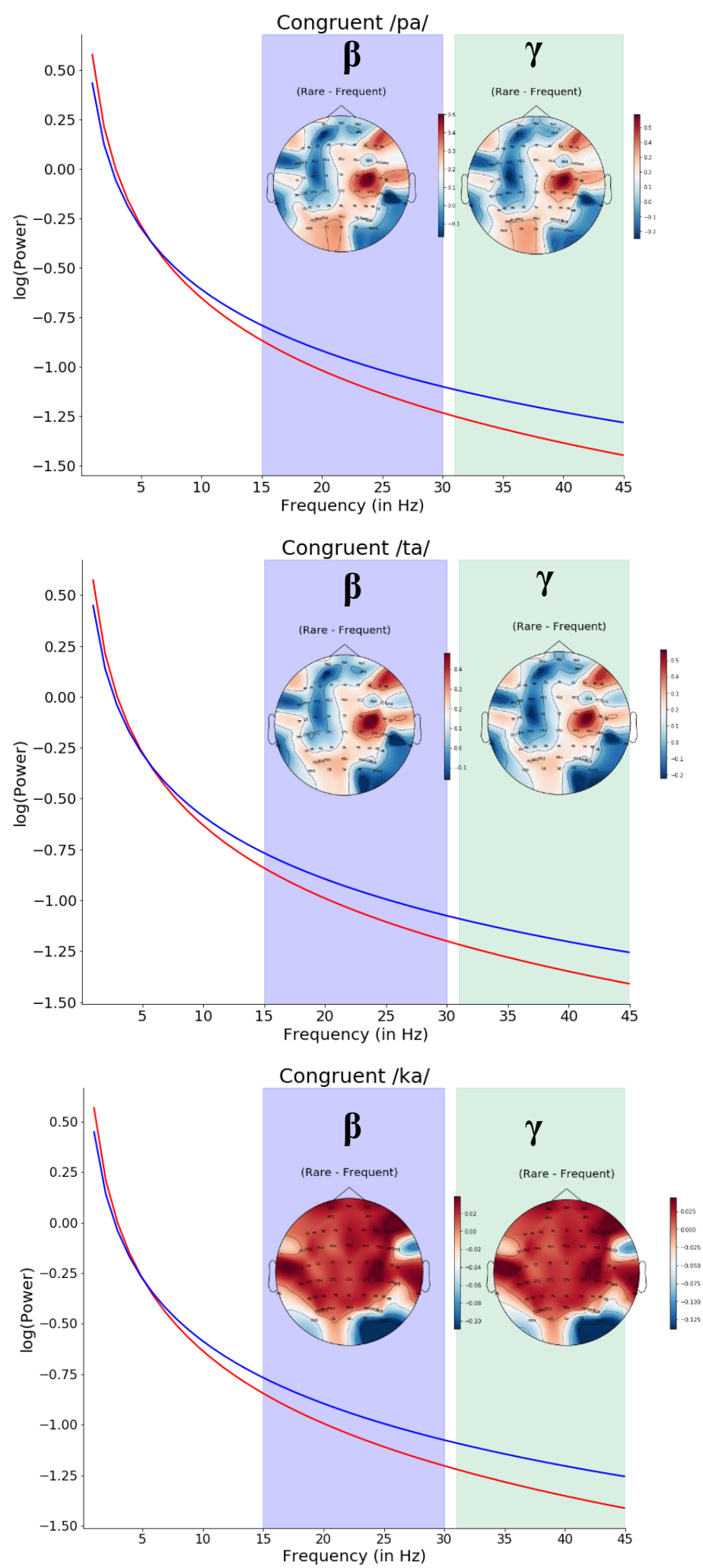

(b) Post-stimulus $800 \mathrm{~ms}$
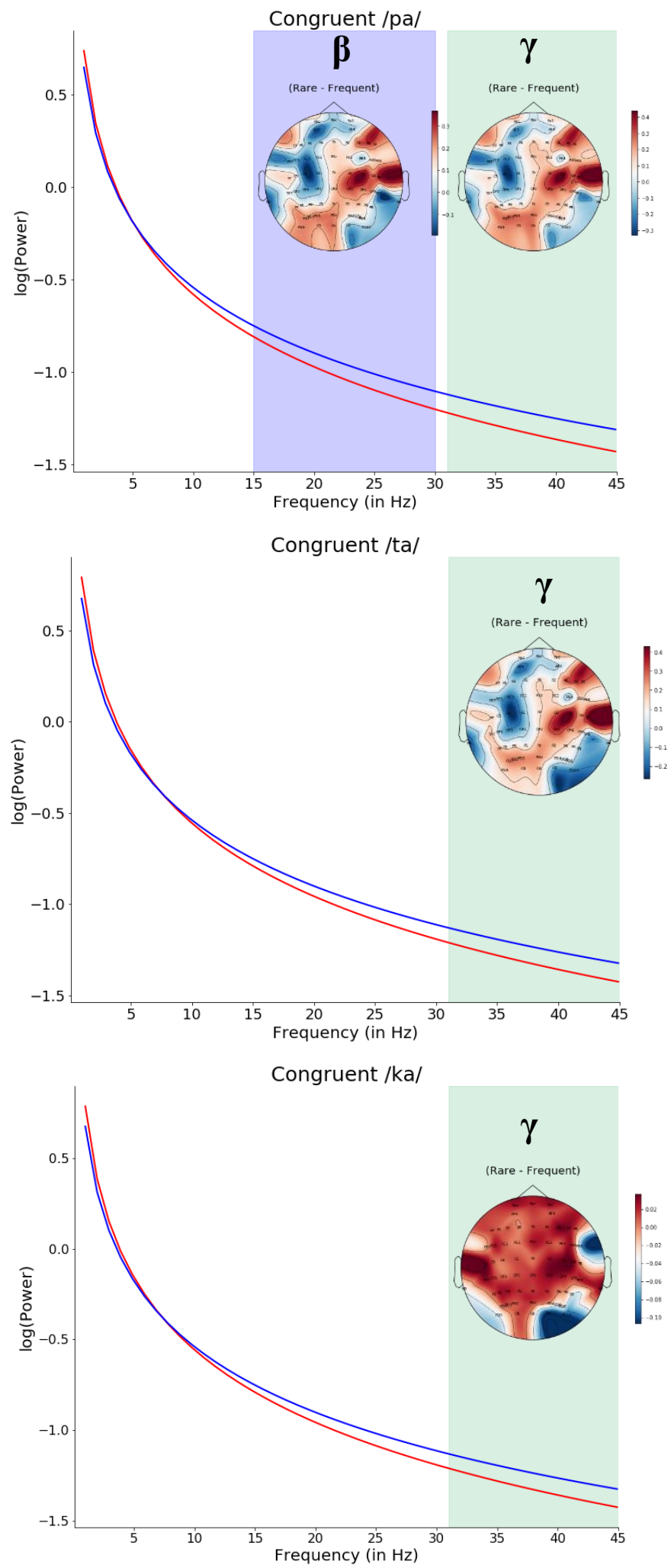

Supplementary Figure 2 


\section{Periodic Distributions}

(a) Pre-stimulus $800 \mathrm{~ms}$ available under aCC-BY 4.0 International licens(b) Post-stimulus $800 \mathrm{~ms}$
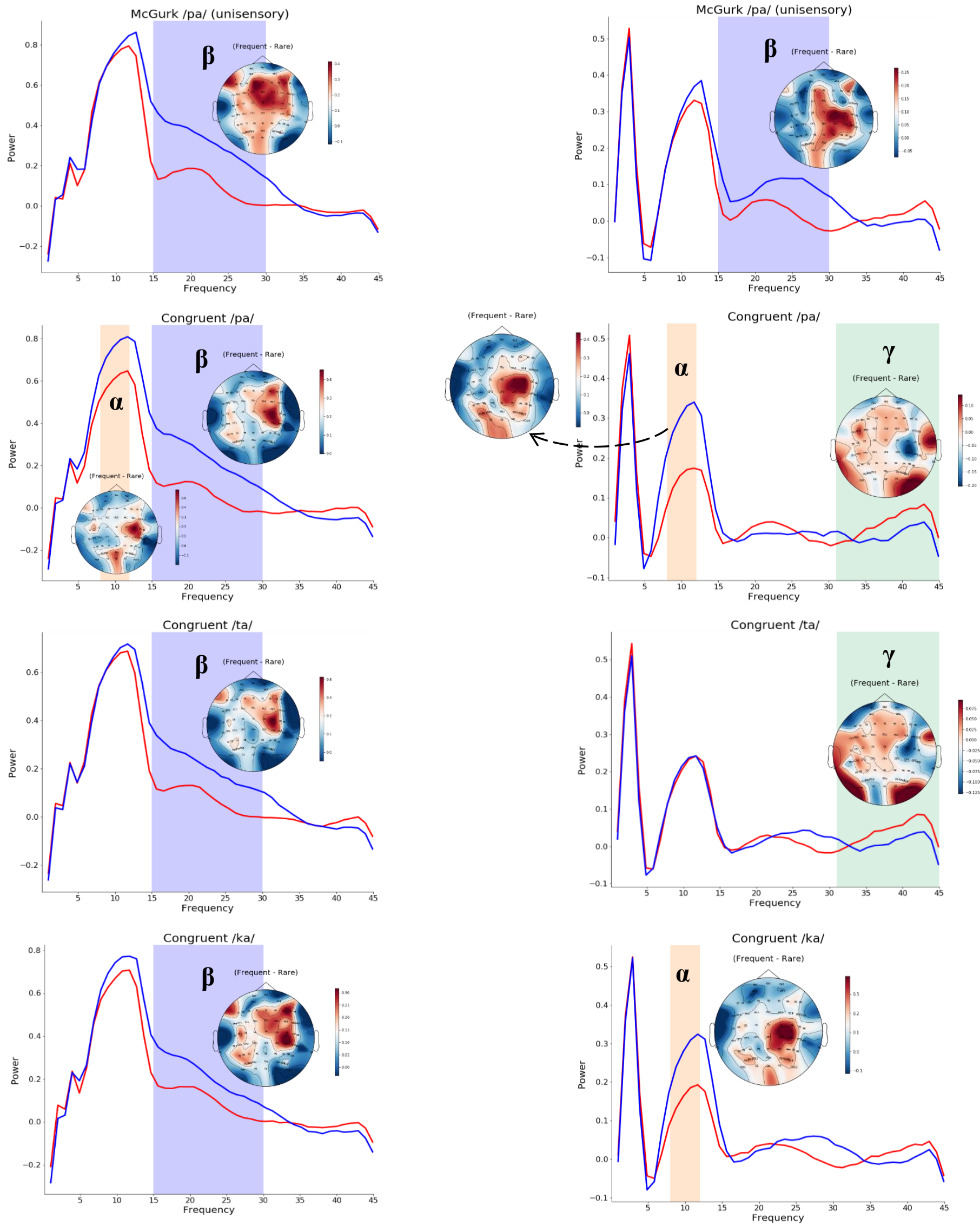


\section{Aperiodic Distributions}

\section{Inter-trial Variability}

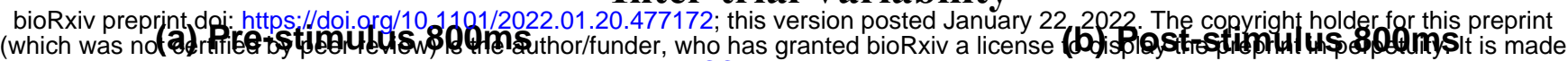
available under aCC-BY 4.0 International license.
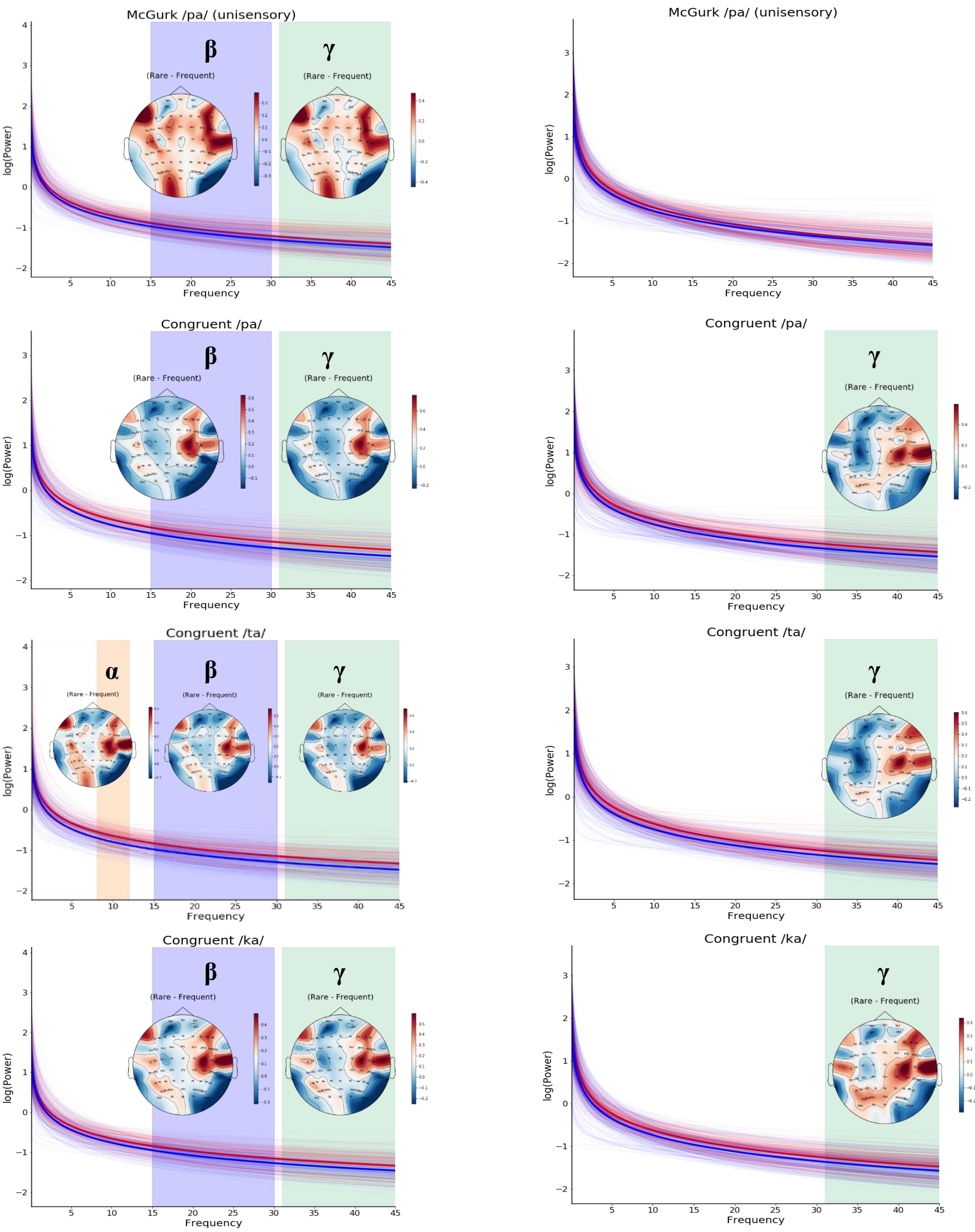


\section{Aperiodic and Periodic Parameters}

bioRxiv preprint doi: https://doi.org/10.1101/2022.01.20.477172; this version posted January 22, 2022. The copyright holder for this preprint (which was not certified by peer review) is the author/funder, who has granted bioRxiv a license to display the preprint in perpetuity. It is made

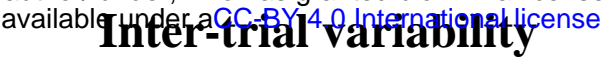

(a) Pre-stimulus $800 \mathrm{~ms}$
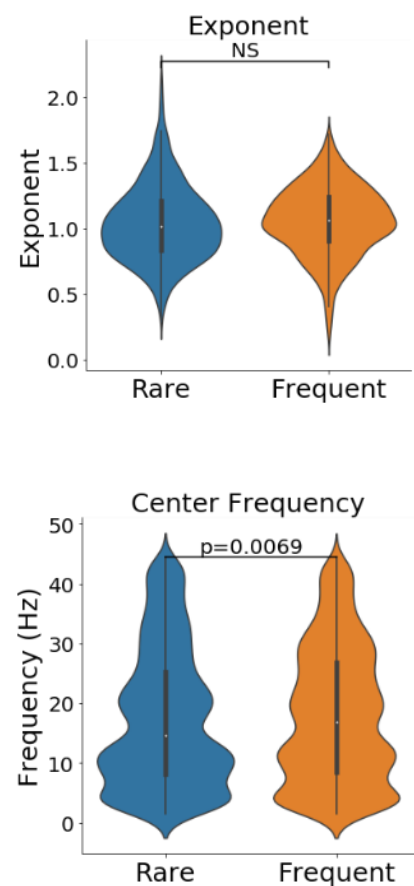
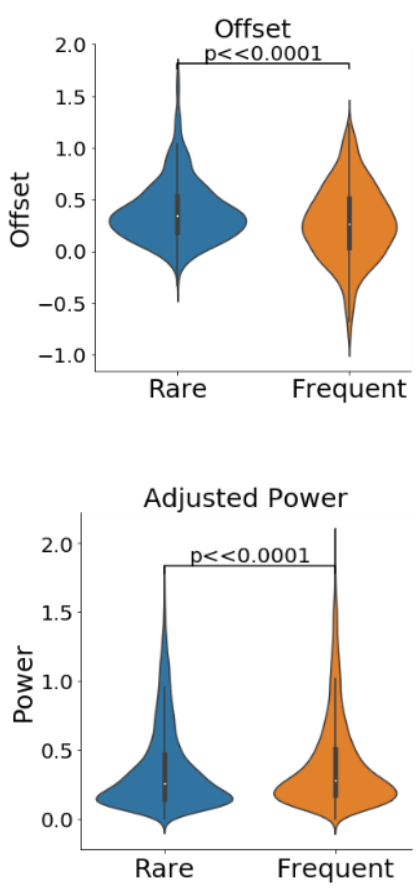

(b) Post-stimulus $800 \mathrm{~ms}$
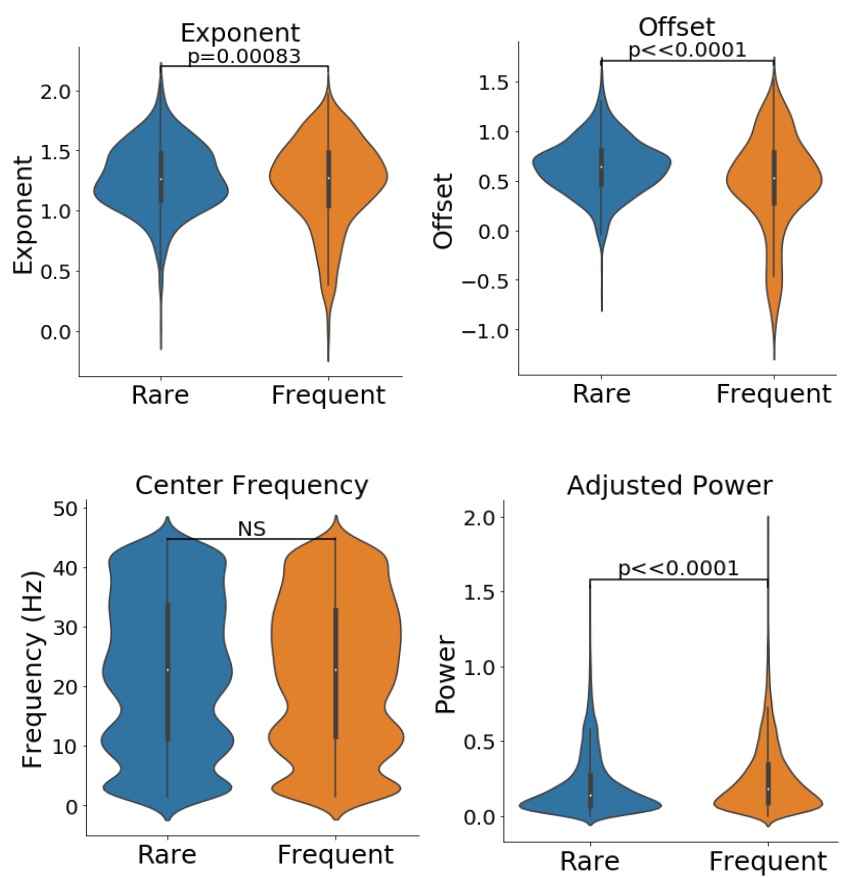
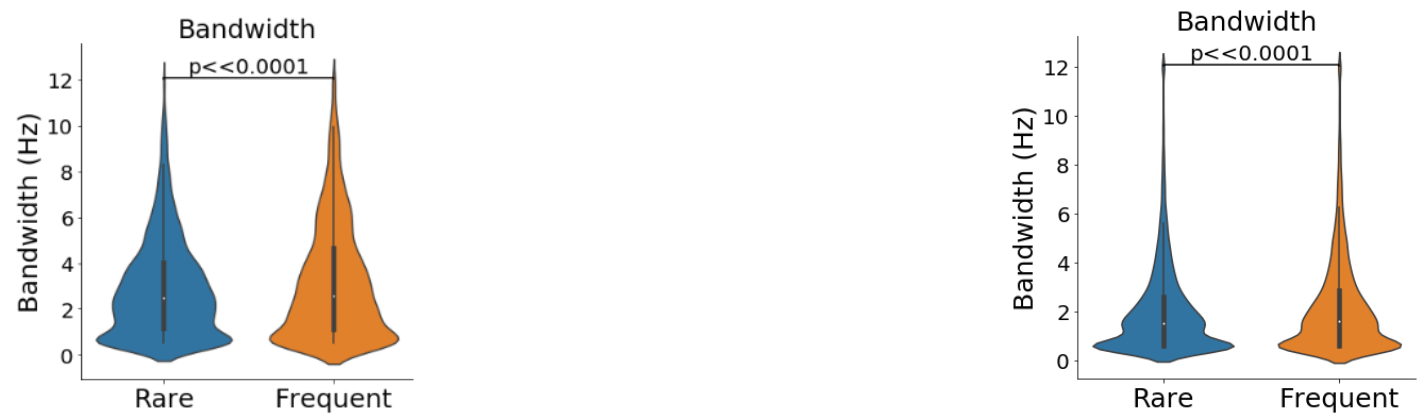

Supplementary Figure 5 


\section{Global Coherence}

bioRxiv preprint doi: https://doi.org/10.1101/2022.01.20.4771.72; this version posted January 22, 2022. The copyright holder for this preprint

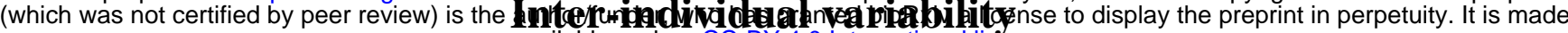
available under aCC-BY 4.0 International license.

(a) Pre-stimulus $800 \mathrm{~ms}$
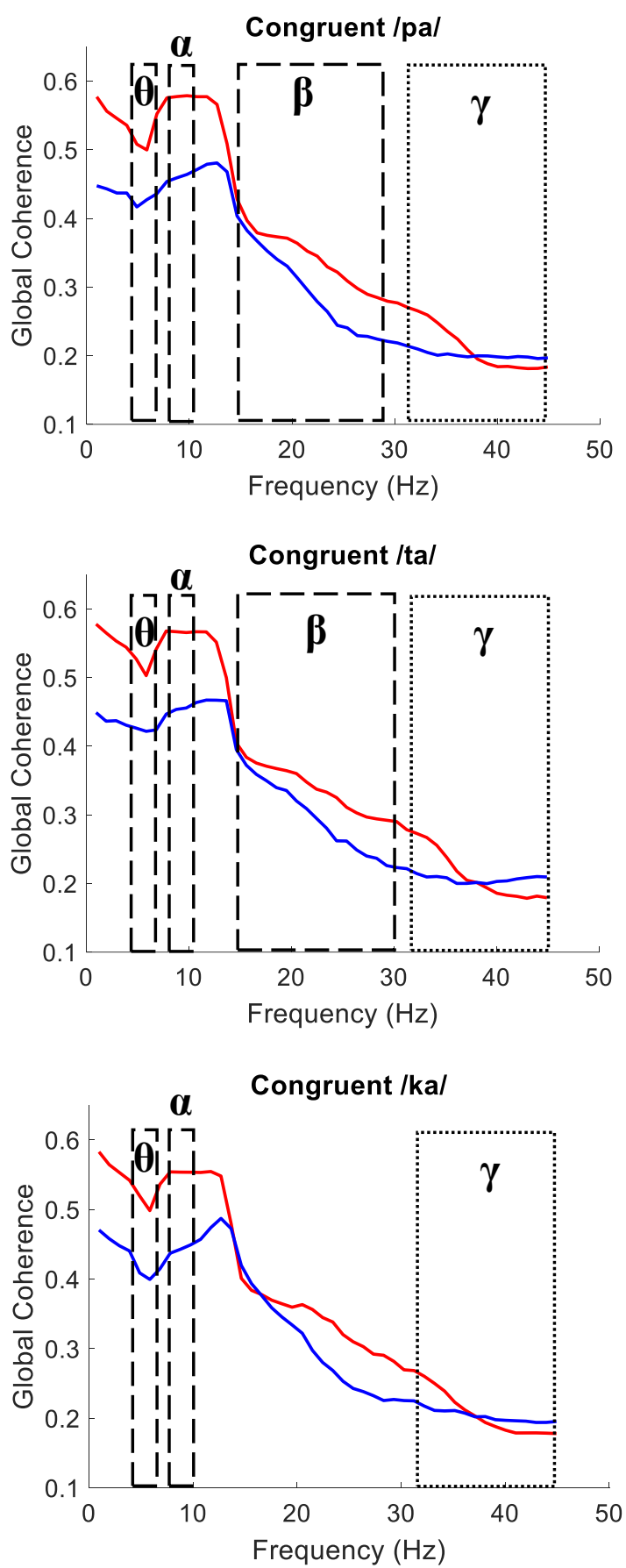

(b) Post-stimulus $800 \mathrm{~ms}$

$<50 \%$ - Rare Perceivers $>50 \%$ - Frequent Perceivers

Congruent /pa/

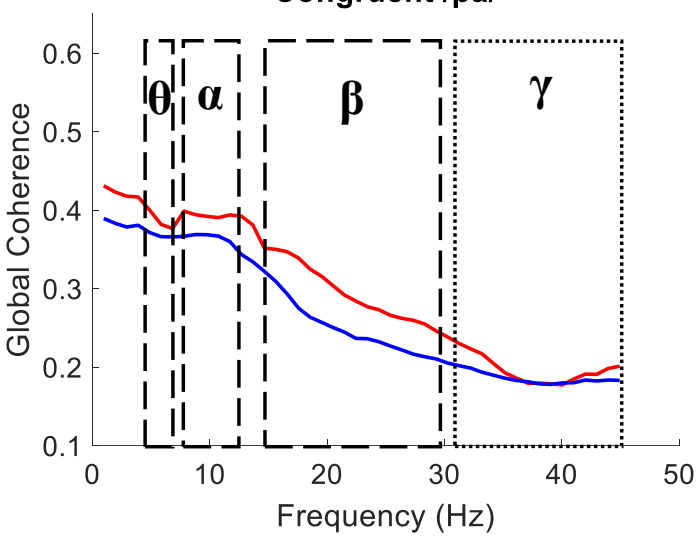

Congruent /ta/

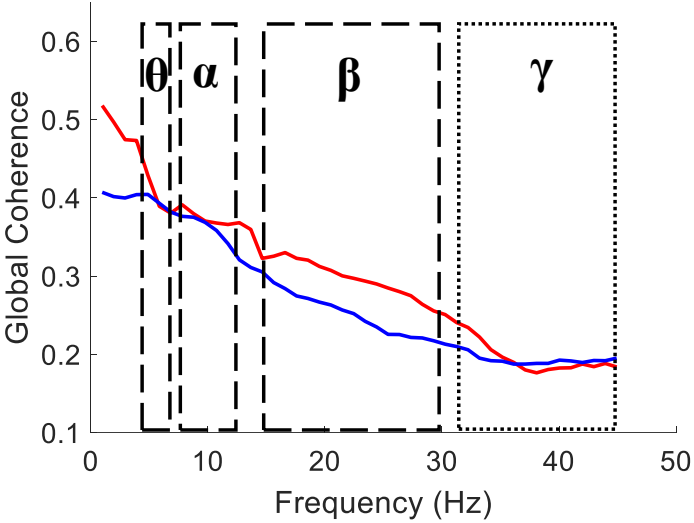

Congruent /ka/

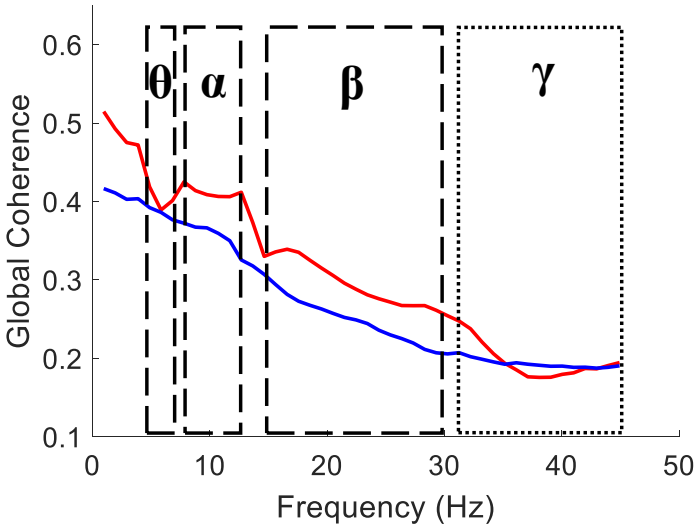

\section{Supplementary Figure 6}


bioRxiv preprint doi: https://doi.org/10.1101/2022.01.20.477172; this version posted January 22, 2022. The copyright holder for this preprint (which was not certified by peer review) is the author/funder, who has granted bioRxiv a license to display the preprint in perpetuity. It is made

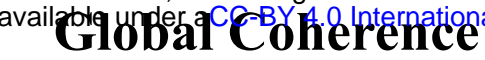

\section{Inter-individual variability}

\section{(a) Pre-stimulus $800 \mathrm{~ms}$}

\begin{tabular}{|ccccc|}
\hline $\begin{array}{c}\text { Stimuli } \\
\mathbf{( 8 0 0} \mathbf{~ m s})\end{array}$ & Theta (4-7 Hz) & Alpha (8-9.5 Hz) & Beta (15-30 Hz) & Gamma (31-45 Hz) \\
\hline Incongruent & 'Significantly higher' & 'Significantly higher' & 'Significantly higher' & 'Significantly lower' \\
McGurk & $Z_{95}=0.42$ & $Z_{95}=0.44$ & $Z_{95}=0.34$ & $Z_{5}=-0.47$ \\
\hline Congruent /pa/ & 'Significantly higher' & 'Significantly higher' & 'Significantly higher' & 'Significantly lower' \\
& $Z_{95}=0.49$ & $Z_{95}=0.39$ & $Z_{95}=0.20$ & $Z_{5}=-0.56$ \\
\hline Congruent /ta/ & 'Significantly higher' & 'Significantly higher' & 'Significantly higher' & 'Significantly lower' \\
& $Z_{95}=0.48$ & $Z_{95}=0.78$ & $Z_{95}=0.32$ & $Z_{5}=-0.44$ \\
\hline Congruent /ka/ & 'Significantly higher' & 'Significantly higher' & 'Insignificant' & 'Significantly lower' \\
& $Z_{95}=0.44$ & $Z_{95}=0.76$ & & $Z_{5}=-0.68$ \\
\hline
\end{tabular}

\section{(b) Post-stimulus 800ms}

\begin{tabular}{|ccccc|}
\hline $\begin{array}{c}\text { Stimuli } \\
\mathbf{( 8 0 0} \mathbf{~ m s})\end{array}$ & Theta (4-7 Hz) & Alpha (8-12 Hz) & Beta (15-30 Hz) & Gamma (31-45 Hz) \\
\hline Incongruent & 'Significantly higher' & 'Significantly higher' & 'Significantly higher' & 'Significantly lower' \\
McGurk & $Z_{95}=0.26$ & $Z_{95}=1.00$ & $Z_{95}=0.56$ & $Z_{5}=-0.40$ \\
\hline Congruent /pa/ & 'Significantly higher' & 'Significantly higher' & 'Significantly higher' & 'Significantly lower' \\
& $Z_{95}=0.31$ & $Z_{95}=0.79$ & $Z_{95}=0.32$ & $Z_{5}=-0.62$ \\
\hline Congruent $/ \mathrm{ta} /$ & 'Significantly higher' & 'Significantly higher' & 'Significantly higher' & 'Significantly lower' \\
& $Z_{95}=0.50$ & $Z_{95}=0.66$ & $Z_{95}=0.42$ & $Z_{5}=-0.47$ \\
\hline Congruent $/ \mathrm{ka} /$ & 'Significantly higher' & 'Significantly higher' & 'Significantly higher' & 'Significantly lower' \\
& $Z_{95}=0.34$ & $Z_{95}=1.02$ & $Z_{55}=-0.43$ \\
\hline
\end{tabular}

\section{Supplementary Table 1}




\section{Global Coherence}

bioRxiv preprint doi: https://doi.org/10.1101/2022.01.20.477172; this version posted January 22, 2022. The copyright holder for this preprint (which was not certified by peer review) is the author/funder, who has granted bioRxiv a license to display the preprint in perpetuity. It is made

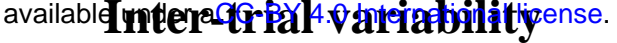

(a) Pre-stimulus $800 \mathrm{~ms}$

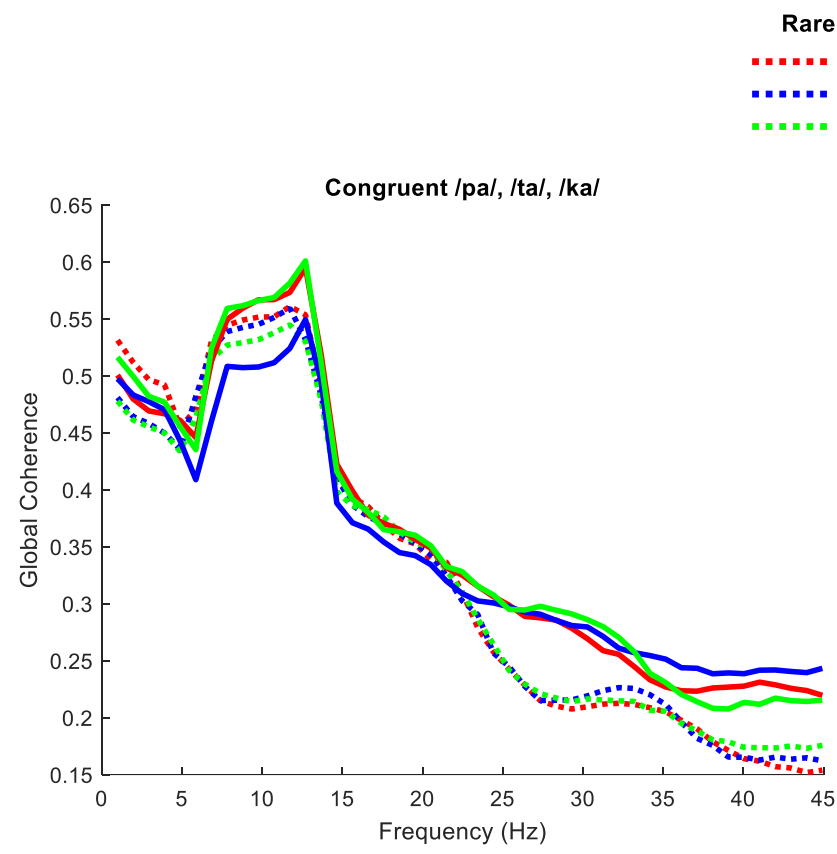

\begin{tabular}{|c|c|c|c|c|}
\hline $\begin{array}{c}\text { Stimuli } \\
\text { (800 ms) }\end{array}$ & $\begin{array}{c}\text { Theta (4-7 } \\
\mathrm{Hz})\end{array}$ & $\begin{array}{c}\text { Alpha (8-12 } \\
\mathrm{Hz})\end{array}$ & $\begin{array}{c}\text { Beta (15-30 } \\
\mathrm{Hz})\end{array}$ & $\begin{array}{c}\text { Gamma (31- } \\
45 \mathrm{~Hz})\end{array}$ \\
\hline $\begin{array}{c}\text { McGurk } \\
\text { /ta/ } \\
\text { (illusory) }\end{array}$ & 'Insignificant' & 'Insignificant' & $\begin{array}{c}\text { 'Significantly } \\
\text { higher' } \\
Z_{95}=0.87\end{array}$ & $\begin{array}{c}\text { 'Significantly } \\
\text { higher' } \\
Z_{95}=0.96\end{array}$ \\
\hline $\begin{array}{l}\text { McGurk } \\
\text { /pa/ (non- } \\
\text { illusory) }\end{array}$ & 'Insignificant' & $\begin{array}{c}\text { 'Significantly } \\
\text { higher' } \\
\text { Z }_{95}=0.92 \\
\end{array}$ & $\begin{array}{c}\text { 'Significantly } \\
\text { higher' } \\
Z_{95}=0.98\end{array}$ & $\begin{array}{c}\text { 'Significantly } \\
\text { higher' } \\
\text { Z }_{95}=0.76\end{array}$ \\
\hline $\begin{array}{l}\text { Congruent } \\
\text { /pa/ }\end{array}$ & 'Insignificant' & 'Insignificant' & $\begin{array}{c}\text { 'Significantly } \\
\text { higher' } \\
\text { Z }_{95}=0.89\end{array}$ & $\begin{array}{c}\text { 'Significantly } \\
\text { higher' } \\
\text { Z }_{95}=0.95\end{array}$ \\
\hline $\begin{array}{c}\text { Congruent } \\
\text { /ta/ }\end{array}$ & 'Insignificant' & $\begin{array}{c}\text { 'Significantly } \\
\text { higher' } \\
\text { Z }_{95}=0.59\end{array}$ & $\begin{array}{c}\text { 'Significantly } \\
\text { higher' } \\
\text { Z }_{95}=0.52\end{array}$ & $\begin{array}{c}\text { 'Significantly } \\
\text { higher' } \\
\text { Z }_{95}=0.55\end{array}$ \\
\hline $\begin{array}{c}\text { Congruent } \\
\text { /ka/ }\end{array}$ & 'Insignificant' & 'Insignificant' & $\begin{array}{c}\text { 'Significantly } \\
\text { higher' } \\
Z_{95}=0.97\end{array}$ & $\begin{array}{c}\text { 'Significantly } \\
\text { higher' } \\
\text { Z }_{95}=0.95\end{array}$ \\
\hline
\end{tabular}

(b) Post-stimulus $800 \mathrm{~ms}$
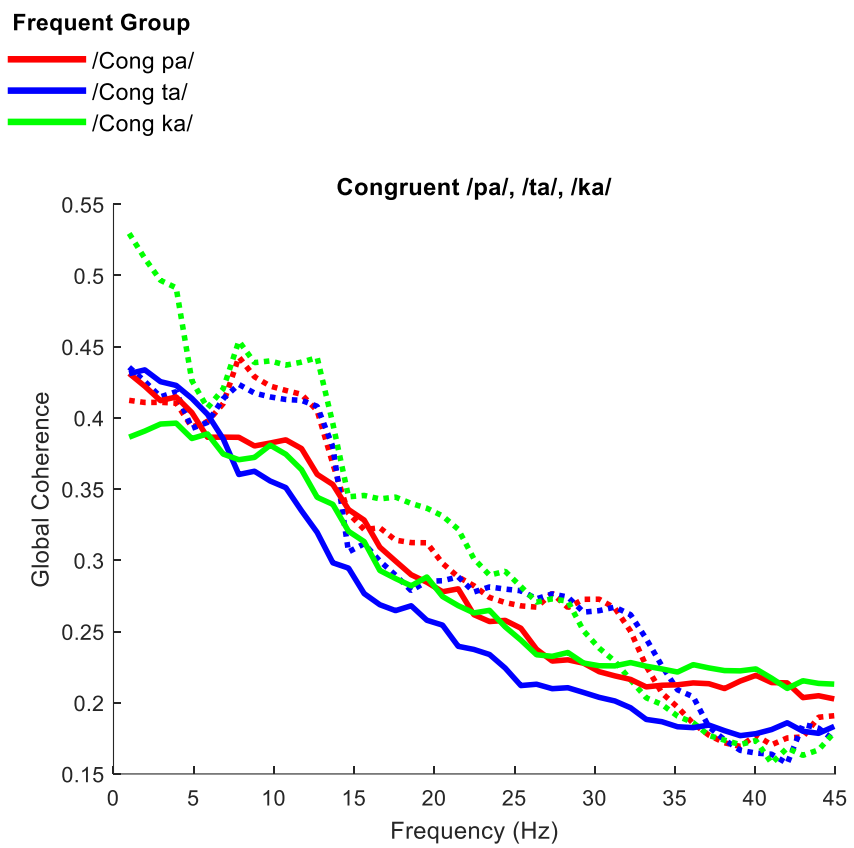

\begin{tabular}{|c|c|c|c|c|}
\hline $\begin{array}{c}\text { Stimuli } \\
(800 \mathrm{~ms})\end{array}$ & $\begin{array}{c}\text { Theta (4-7 } \\
\text { Hz) }\end{array}$ & $\begin{array}{c}\text { Alpha (8-12 } \\
\mathrm{Hz})\end{array}$ & $\begin{array}{c}\text { Beta (15-30 } \\
\mathrm{Hz})\end{array}$ & $\begin{array}{c}\text { Gamma (31- } \\
45 \mathrm{~Hz})\end{array}$ \\
\hline $\begin{array}{c}\text { McGurk } \\
\text { /ta/ } \\
\text { (illusory) }\end{array}$ & 'Insignificant' & $\begin{array}{c}\text { 'Significantly } \\
\text { higher' } \\
Z_{95}=0.61\end{array}$ & $\begin{array}{c}\text { 'Significantly } \\
\text { higher' } \\
Z_{95}=0.57\end{array}$ & 'Insignificant' \\
\hline $\begin{array}{l}\text { McGurk } \\
\text { /pa/ (non- } \\
\text { illusory) }\end{array}$ & $\begin{array}{l}\text { 'Significantly } \\
\text { higher' } \\
\text { Z }_{95}=0.62\end{array}$ & $\begin{array}{c}\text { 'Significantly } \\
\text { higher' } \\
\text { Z }_{95}=0.63\end{array}$ & $\begin{array}{c}\text { 'Significantly } \\
\text { higher' } \\
Z_{95}=0.68\end{array}$ & $\begin{array}{c}\text { 'Significantly } \\
\text { higher' } \\
Z_{95}=0.80\end{array}$ \\
\hline $\begin{array}{l}\text { Congruent } \\
\text { /pa/ }\end{array}$ & 'Insignificant' & $\begin{array}{c}\text { 'Significantly } \\
\text { higher' } \\
Z_{95}=0.87\end{array}$ & $\begin{array}{c}\text { 'Significantly } \\
\text { higher' } \\
Z_{95}=0.51\end{array}$ & 'Insignificant' \\
\hline $\begin{array}{c}\text { Congruent } \\
/ \mathrm{ta} /\end{array}$ & 'Insignificant' & 'Insignificant' & $\begin{array}{c}\text { 'Significantly } \\
\text { higher' } \\
Z_{95}=0.42\end{array}$ & $\begin{array}{c}\text { 'Significantly } \\
\text { higher' } \\
Z_{95}=0.54\end{array}$ \\
\hline $\begin{array}{c}\text { Congruent } \\
\text { /ka/ }\end{array}$ & $\begin{array}{c}\text { Significantly } \\
\text { higher' } \\
Z_{95}=0.58\end{array}$ & $\begin{array}{c}\text { 'Significantly } \\
\text { higher' } \\
Z_{95}=0.47\end{array}$ & $\begin{array}{c}\text { 'Significantly } \\
\text { higher' } \\
Z_{95}=0.46\end{array}$ & 'Insignificant' \\
\hline
\end{tabular}

\section{Supplementary Figure 7}




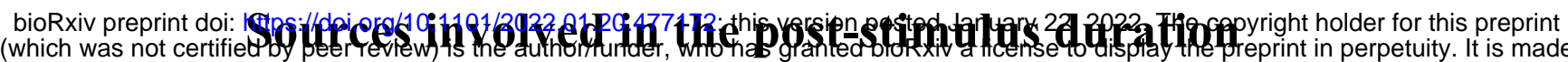
available under aCC-BY 4.0 International license.

\section{(a) For the rare group of perceivers:}

\begin{tabular}{ccccc}
\hline $\mathbf{X}$ & $\mathbf{Y}$ & $\mathbf{Z}$ & Brodmann area & Region \\
\hline-32.929 & 58.492 & 6.526 & Left-BA-10 & Left anterior prefrontal cortex \\
33.354 & -78.130 & 61.808 & Right-BA-7 & Right visual motor \\
-38.513 & -2.808 & 66.833 & Left-BA-6 & Left premotor + supplementary motor cortex \\
50.411 & 47.544 & 9.487 & Right-BA-46 & Right dorsolateral prefrontal cortex (lateral) \\
64.949 & 13.164 & 27.377 & Right-BA-9 & Right dorsolateral prefrontal cortex (dorsal) \\
64.569 & 10.316 & 4.958 & Right-BA-44 & Right Broca's opercularis \\
-57.167 & 23.809 & 17.108 & Left-BA-45 & Left Broca's triangularis \\
35.409 & 60.045 & 7.795 & Right-BA-10 & Right anterior prefrontal cortex \\
68.046 & -8.981 & 3.192 & Right-BA-22 & Right superior temporal gyrus \\
63.598 & -54.244 & 39.634 & Right-BA-39 & Right angular gyrus \\
\hline
\end{tabular}

(b) For the frequent group of perceivers:

\begin{tabular}{ccccc}
\hline $\mathbf{X}$ & $\mathbf{Y}$ & $\mathbf{Z}$ & Brodmann area & Region \\
\hline-57.207 & 24.973 & 18.062 & Left-BA-45 & Left Broca's triangularis \\
34.975 & 60.339 & 8.33 & Right-BA-10 & Right anterior prefrontal cortex \\
33.197 & -78.360 & 61.779 & Right-BA-7 & Right visual motor \\
-39.849 & -2.764 & 65.615 & Left-BA-6 & Left premotor + supplementary motor area \\
68.133 & -8.955 & 2.795 & Right-BA-22 & Right superior temporal gyrus \\
-33.499 & 58.579 & 7.663 & Left-BA-10 & Left anterior prefrontal cortex \\
49.522 & 48.710 & 8.381 & Right-BA-46 & Right dorsolateral prefrontal cortex (lateral) \\
64.933 & 11.424 & 14.329 & Right-BA-44 & Right Broca's opercularis \\
63.814 & -54.111 & 39.725 & Right-BA-39 & Right angular gyrus \\
\hline
\end{tabular}




\section{Post-stimulus}

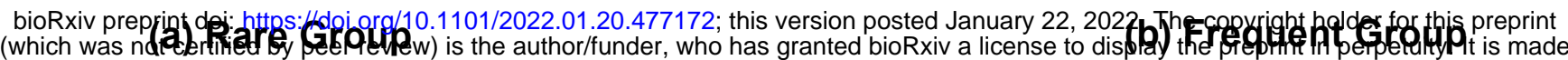
(Theta band) available under aCC-BY 4.0 International license.

(Theta band)

L
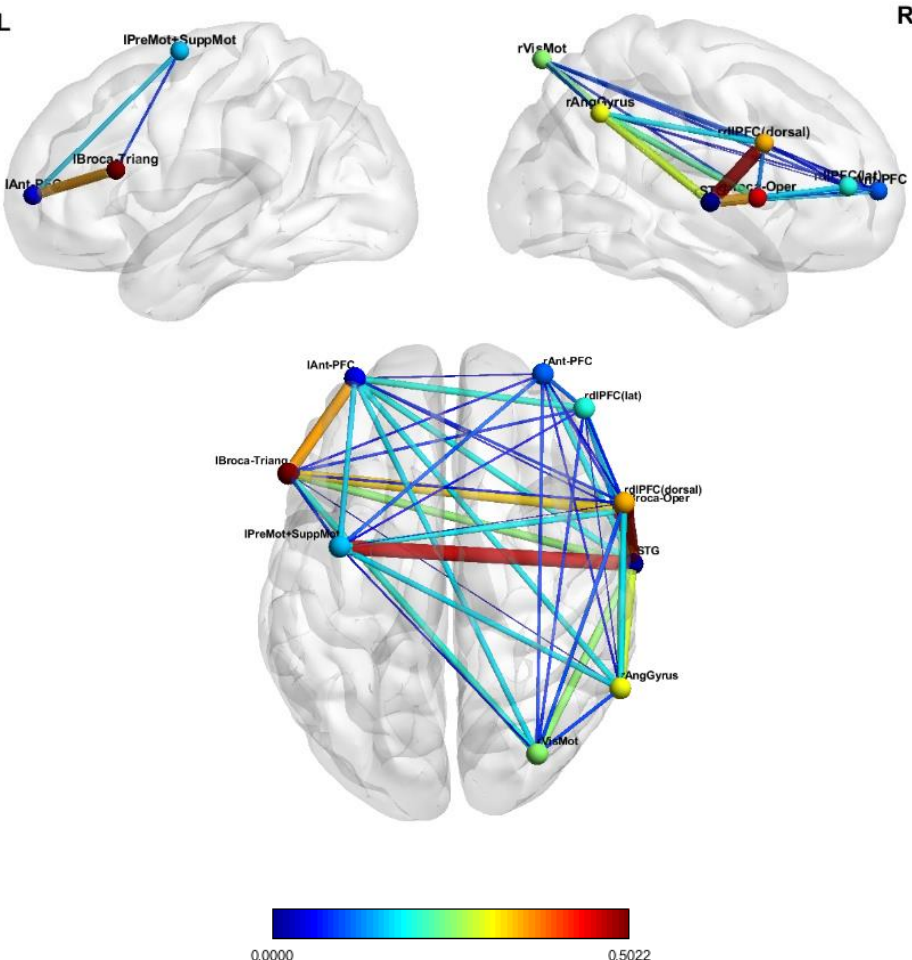

(c) Rare Group

(Alpha band)

L
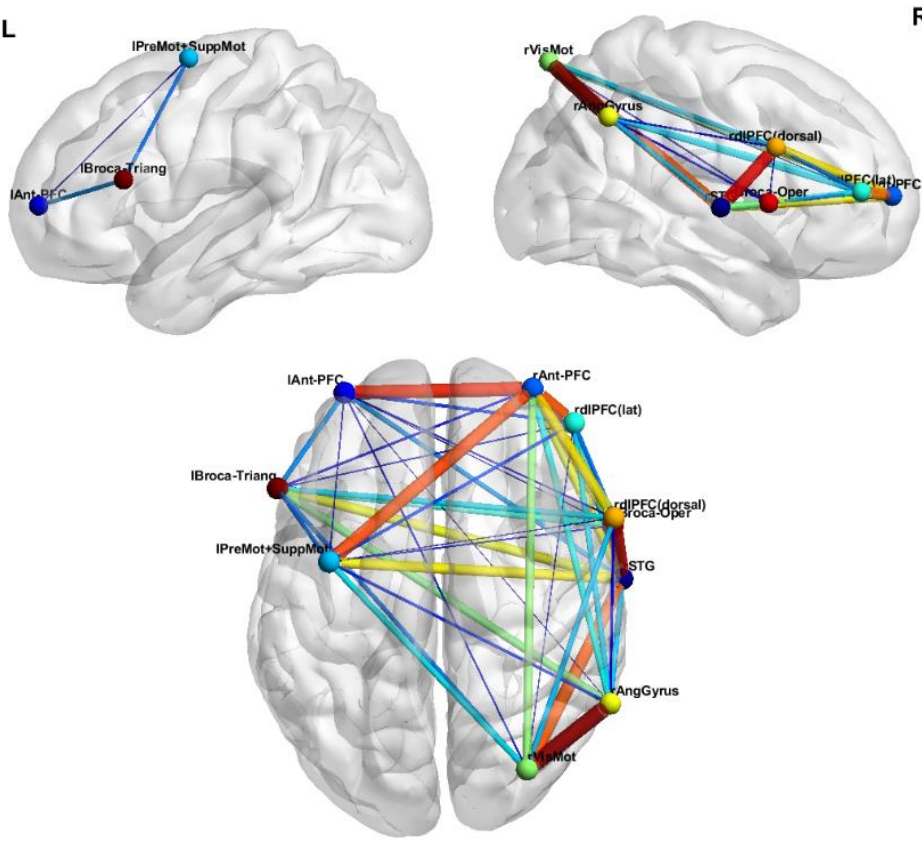

0.0001
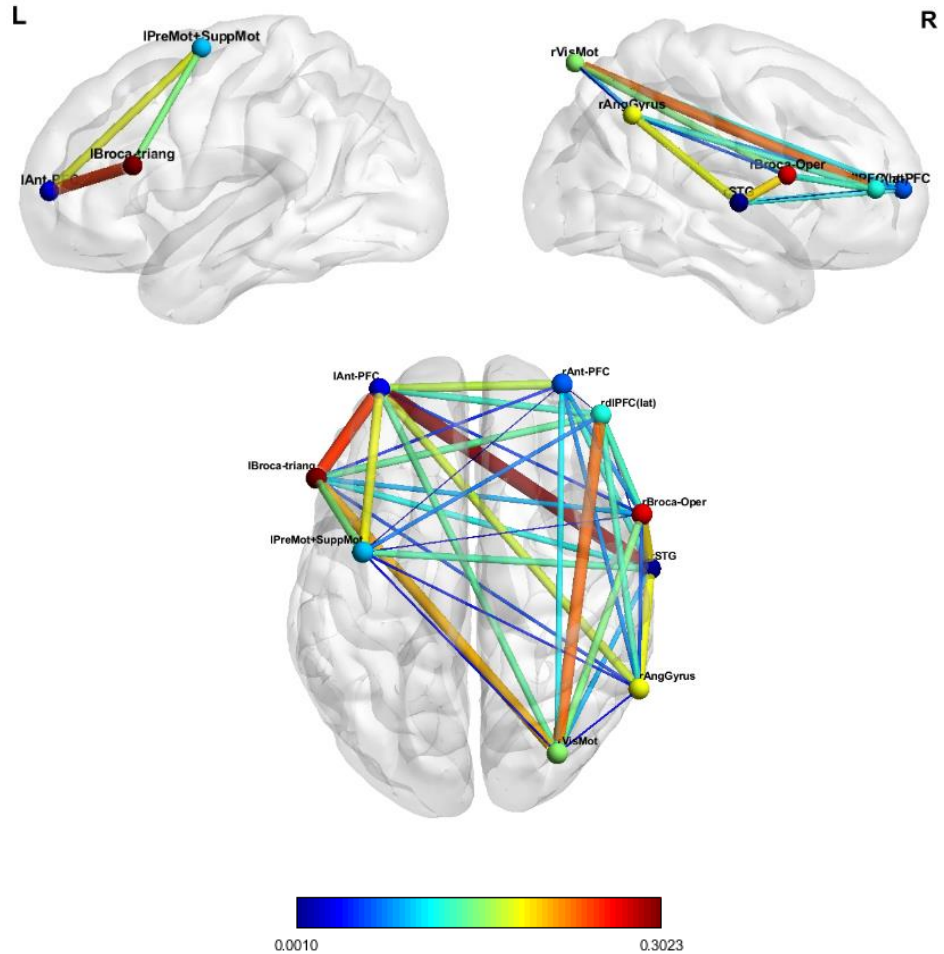

(d) Frequent Group (Alpha band)
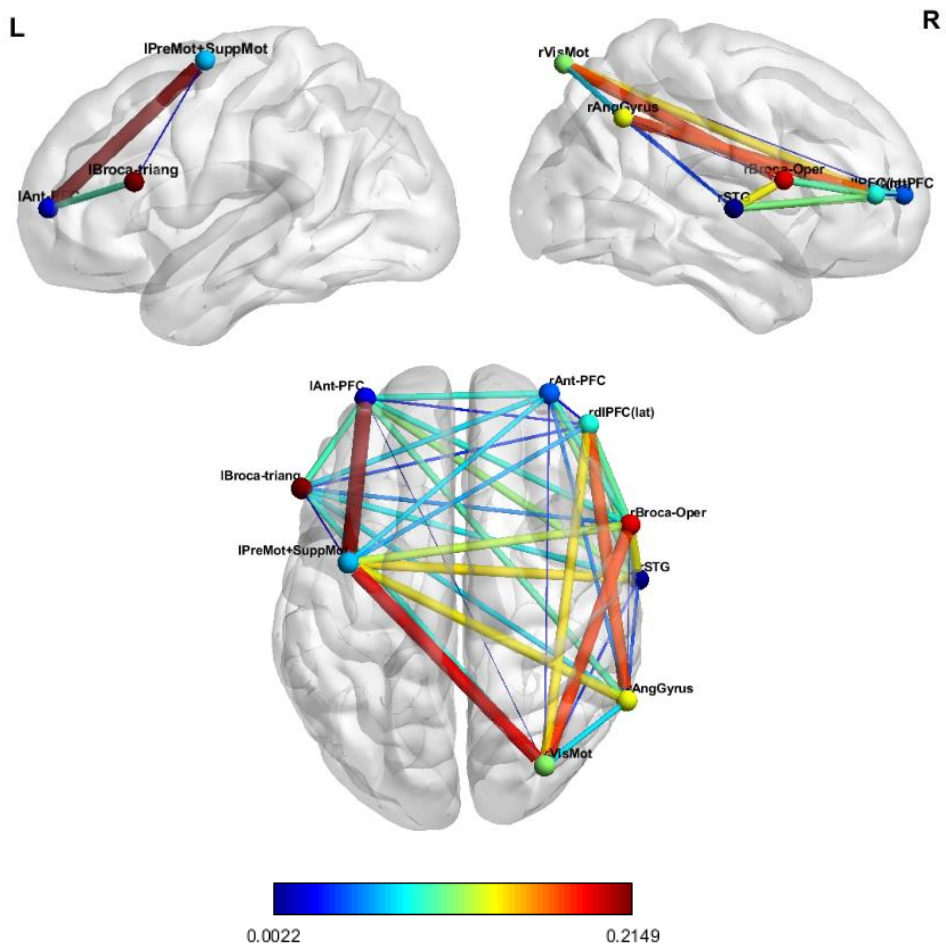


\section{Post-stimulus}

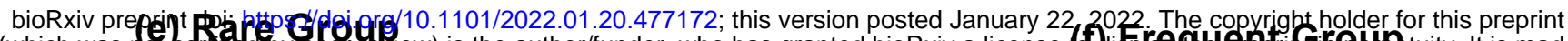
(which was not certifed by peer (Beta band)

(Beta band)
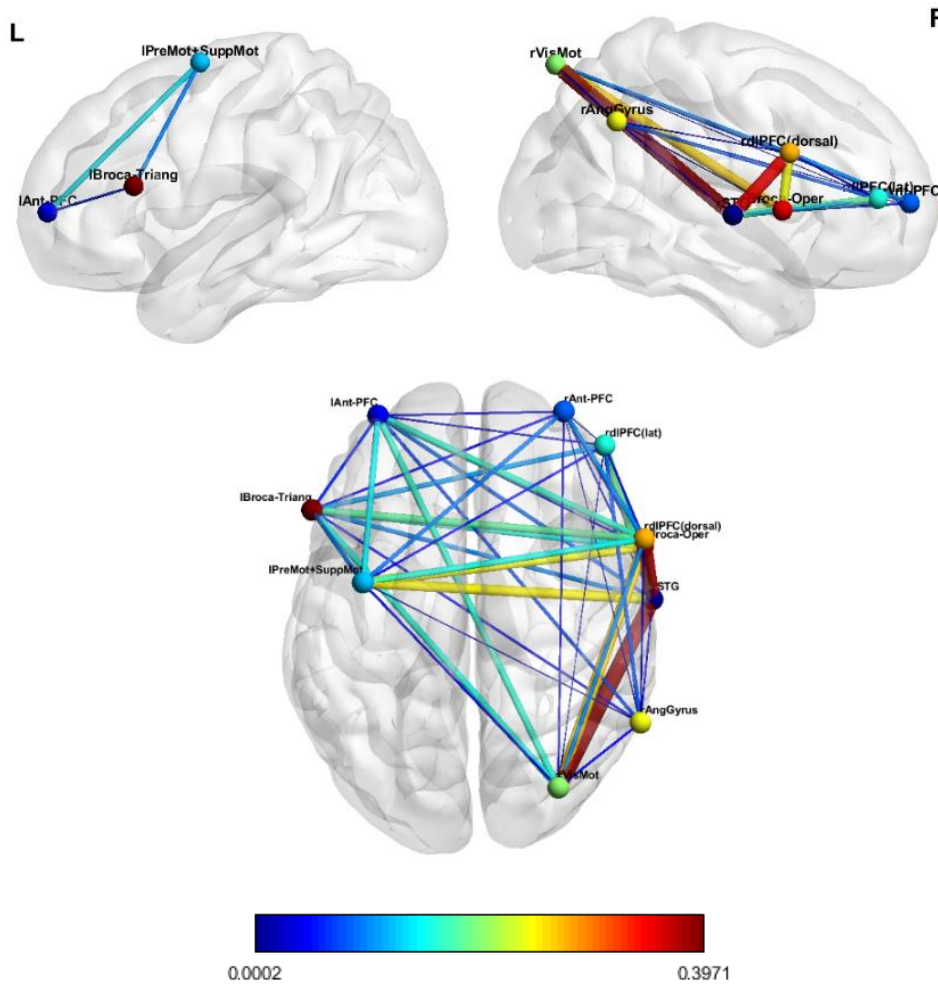

(g) Rare Group

(Gamma band)

L
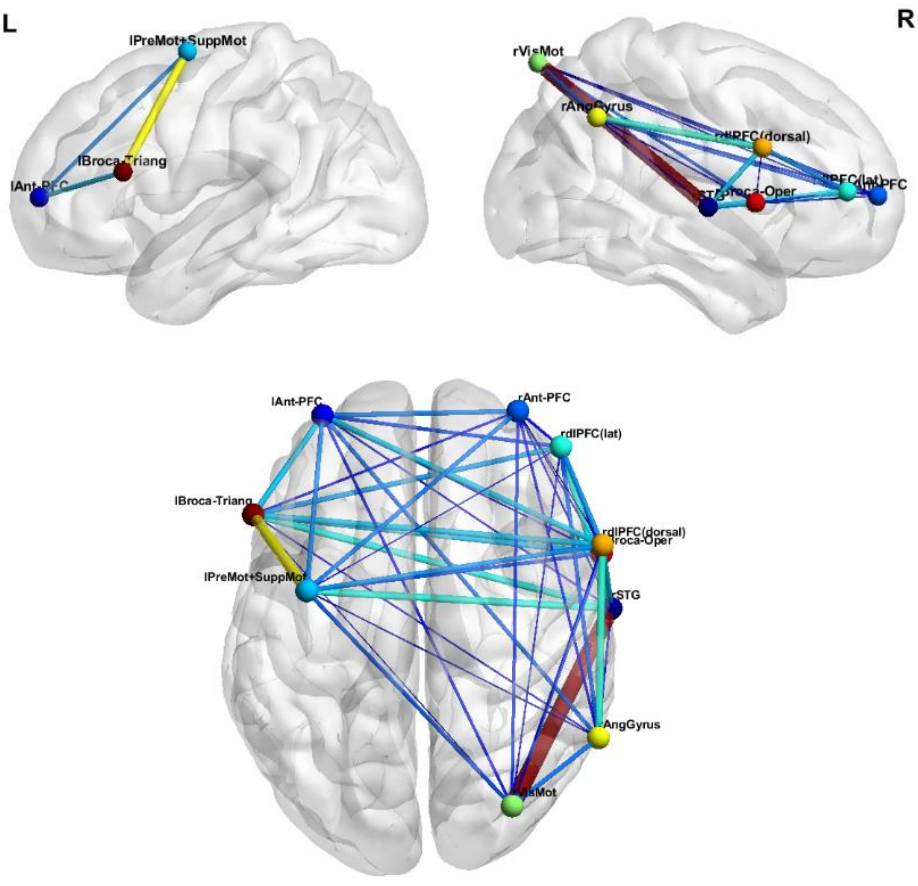

0.0042
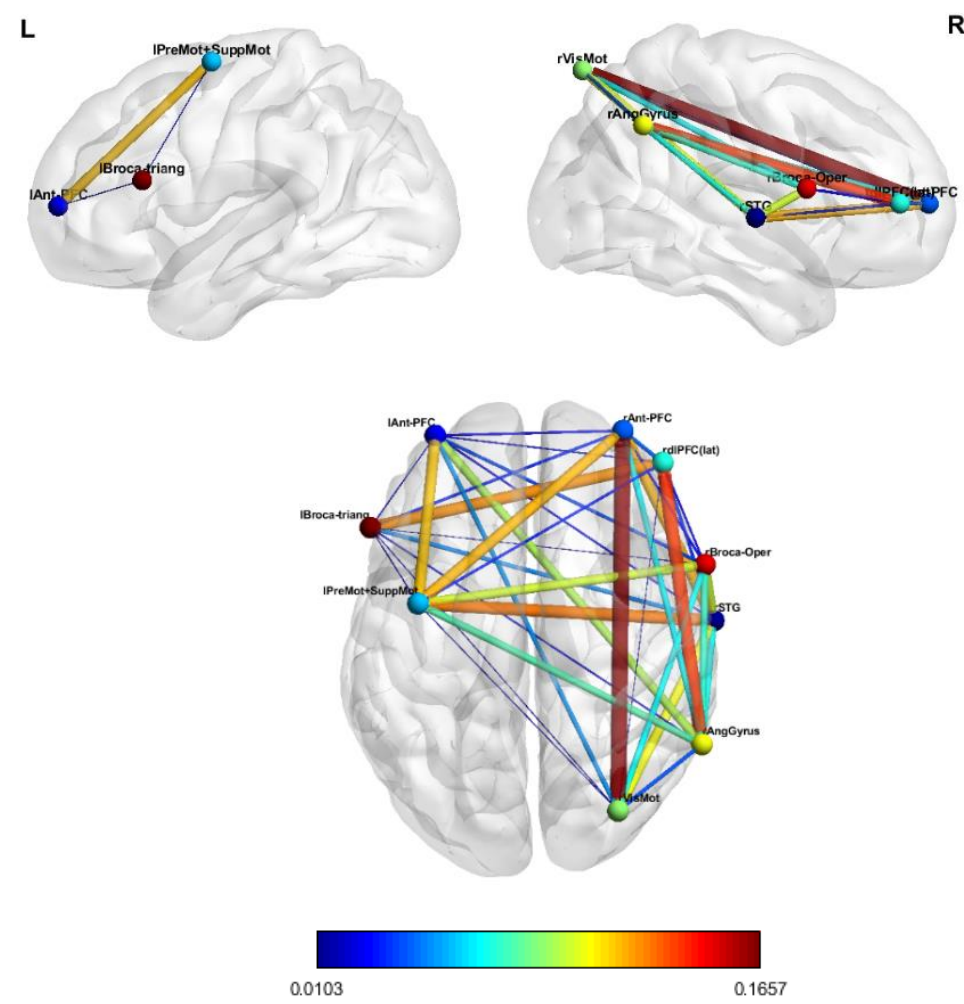

(h) Frequent Group

(Gamma band)
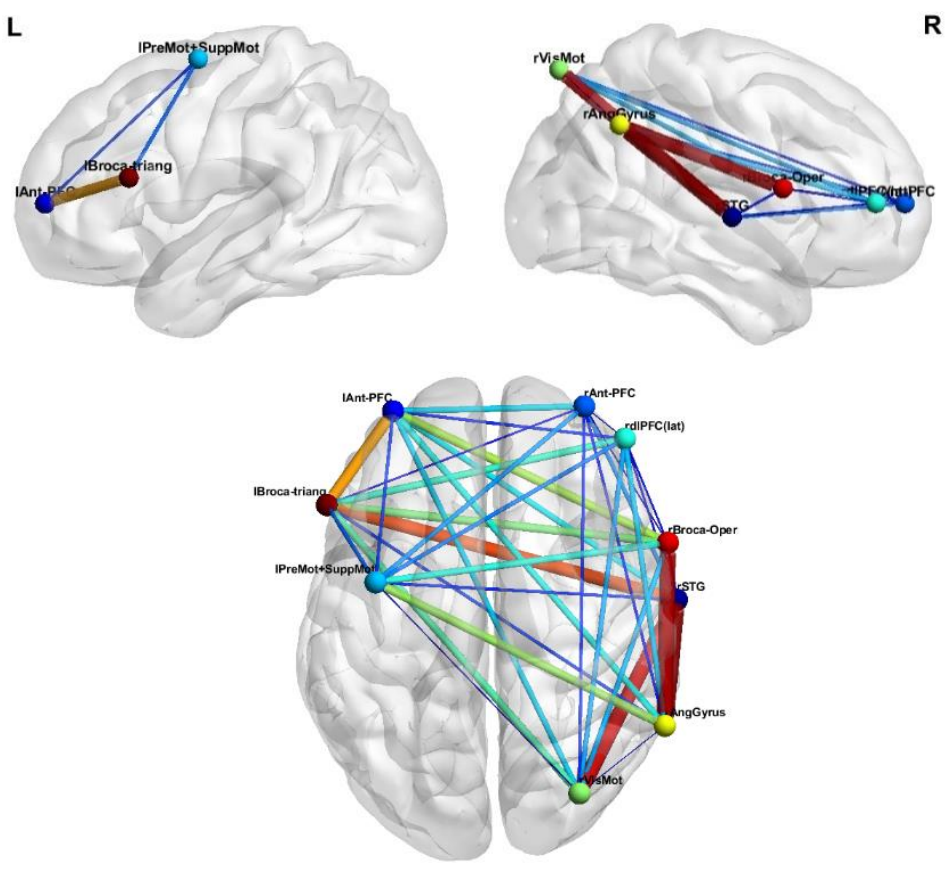

0.0056

. 